\title{
Priority Setting and Integrated Assessment of Transportation System Vulnerabilities to Deep Uncertainties
}

\author{
A Dissertation \\ Presented to the Faculty of the School of Engineering and Applied Science \\ University of Virginia
}

In Partial Fulfillment

of the requirements for the degree of

Doctor of Philosophy in Systems Engineering

Haowen You

December 2013 


\section{Approval}

This dissertation is submitted in partial fulfillment of the

requirements for the degree of Doctor of Philosophy in Systems Engineering

Haowen You

Prof. James H. Lambert, Dissertation Advisor

Prof. Peter A. Beling, Committee Member

Prof. Garrick E. Louis, Committee Chair

Prof. Stephanie A. Guerlain, Committee Member

Prof. Andres H. Clarens, Committee Member

James H. Aylor, Dean, School of Engineering and Applied Science 


\begin{abstract}
Deep uncertainty suggests situations in which the parties to a decision lack consensus on 1) the structural models to describe interactions among a system's variables, 2) the stochastic models to represent uncertainty about key parameters in the models, and 3) the methodologies to value the desirability of alternative outcomes. Deep uncertainties need to be addressed in strategic planning and priority-setting for transportation systems. In this context, traditional economic analysis and risk analysis of particular uncertainties can be prohibitive due to sparse data, complex models, and unforeseen interactions of climate change with other stressors. Nevertheless, decision-makers and planners need to assess the system vulnerability and allocate resources to address the range of deep uncertainties. Recent work in this topic has quantified the influence of deep uncertainties from a single management perspective, via expert elicitation to update the priorities of strategic resources for climate and non-climate stressors. There remains a need to address the multiple perspectives of priority-setting. For transportation agencies, the key perspectives include asset management, project selection, policy making, demographic/geographic equity, and others. This dissertation identifies and quantifies the influence of climate change combining with other sources of uncertainty to priority-setting in several perspectives. The approach adopts methods of multiple criteria decision analysis and scenario-based planning that have been used by agencies for long-range transportation plans. The approach is demonstrated with the Hampton Roads region of the Commonwealth of Virginia, USA. The results include that climate change combining with increased travel demand is relatively influential to priority-setting across scenarios that include economic downturn, ecological change, wear and tear, and climate change alone. The influential scenarios are shown to differ by perspective.
\end{abstract}




\section{Keywords}

Vulnerability assessment; Risk analysis; Transportation planning; Multicriteria decision analysis; Scenario planning; Integrated analysis; Resource priority setting; Deep uncertainties 


\section{Acknowledgements}

I would like to express my sincerest gratitude to my advisor, Professor James H. Lambert for his guidance and support throughout my doctoral program. I am also grateful to my dissertation committee: Professor Peter A. Beling, Professor Stephanie A. Guerlain, Professor Garrick E. Louis of the Department of Systems and Information Engineering, and Professor Andres F. Clarens of the Department of Civil and Environmental, for their invaluable knowledge and assistance.

I am grateful to the faculty and staff of the Department of Systems and Information Engineering of the University of Virginia for their guidance and the opportunity to pursue this degree. I am grateful to the Federal Highway Administration (FHWA); Virginia Department of Transportation (VDOT); the Virginia Center for Transportation Innovation and Research (VCTIR); the Hampton Roads Transportation Planning Organization (HRTPO); and Hampton Roads Planning District Commission (HRPDC) for their support of this research.

I am grateful to my parents, Zhengxing and Ailin for the support and understanding of all my life. I thank my fiancee, Xinye for providing unrelenting support throughout the development of my research. Finally, I thank my colleagues and friends in the Center for Risk Management of Engineering Systems for their friendship and motivational support. 


\section{Table of Contents}

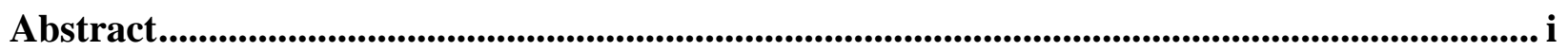

Acknowledgements ............................................................................................................................. iii

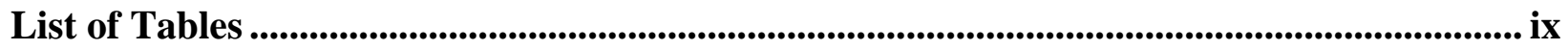

List of Figures............................................................................................................................................. xii

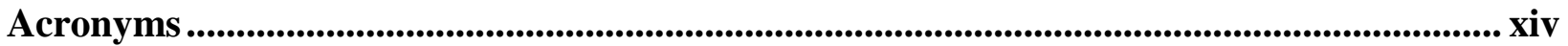

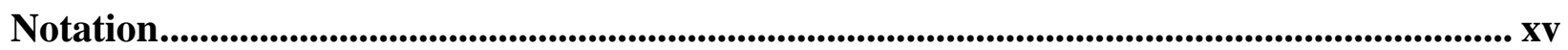

Chapter 1. Introduction ....................................................................................................................................... 1

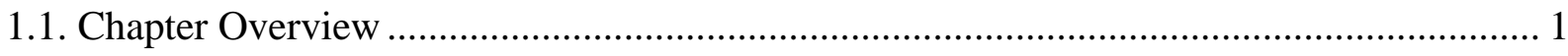

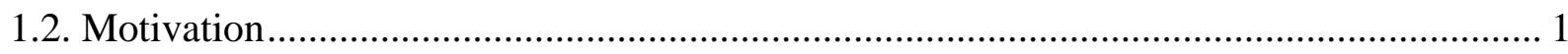

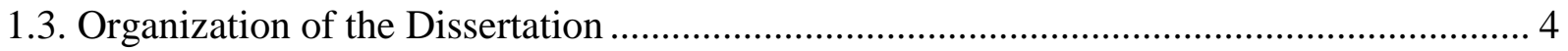

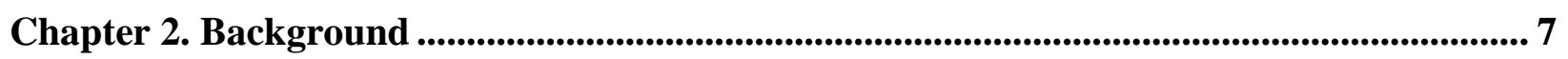

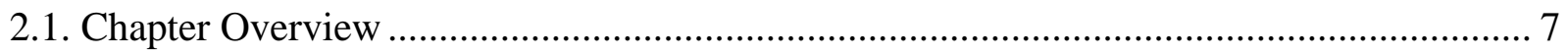

2.2. Climate Change and Impacts on Transportation ................................................. 8

2.3. Transportation Planning under Deep Uncertainties ................................................. 11

2.3.1. The metropolitan planning organization (MPO) ............................................ 11

2.3.2. Transportation planning to address climate change ........................................ 12

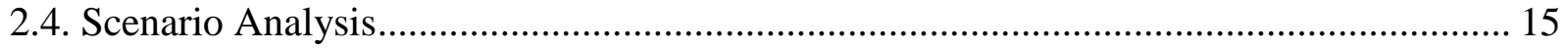

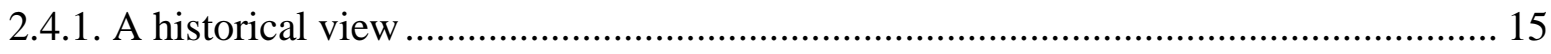

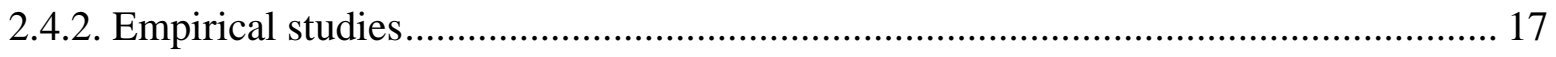

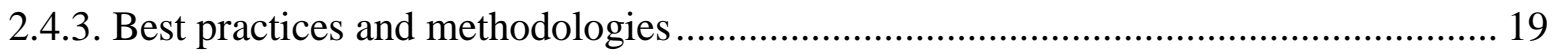




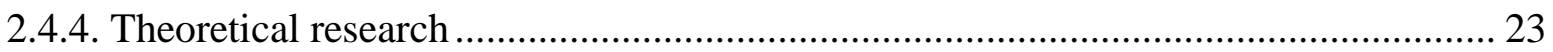

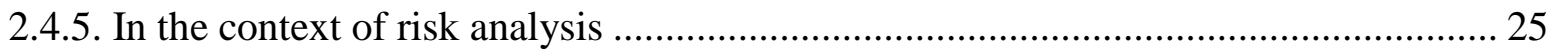

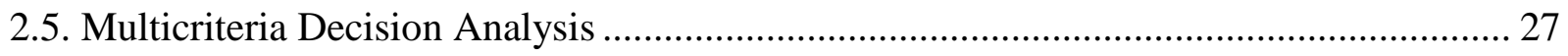

2.5.1. Multiattribute utility theory and multiattribute value theory ..................................... 27

2.5.2. Multicriteria decision analysis in systems analysis and risk analysis........................ 29

2.6. Integration of Multicriteria Decision Analysis and Scenario Analysis ............................ 30

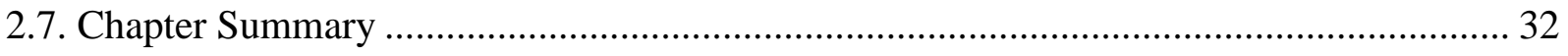

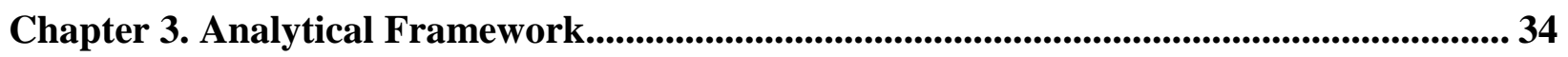

3.1. Chapter Overview ……………………………………........................................... 34

3.2. Multicriteria Priority-Setting on Decision Alternatives...................................................... 35

3.3. Priority-Setting Informed by Scenario Analysis............................................................. 36

3.4. Incremental Adjustment of Value Functions for Scenarios ............................................... 38

3.5. System Vulnerability Assessment via Priority Setting Results ........................................ 40

3.6. Integrated Analysis from Multiple Perspectives................................................................ 43

3.7. On the Implementation of the Analytical Framework ..................................................... 48

3.7.1. The Selection of Multicriteria Decision Analysis Methods....................................... 48

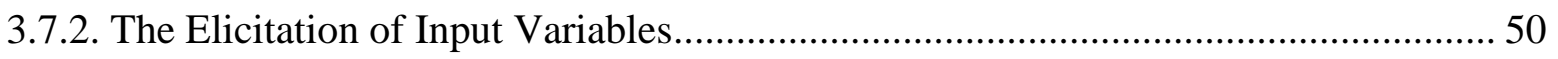

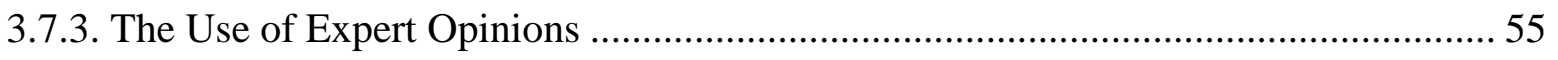

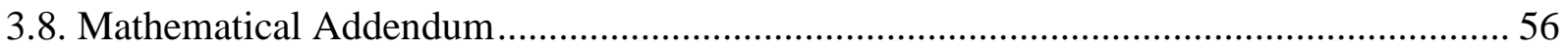

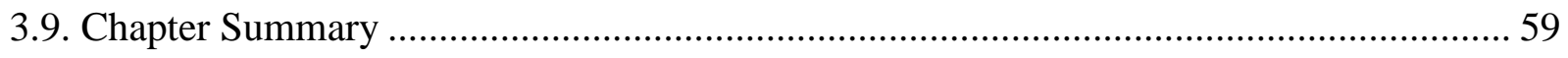

Chapter 4. Implementation and Case Studies.................................................................................. 60

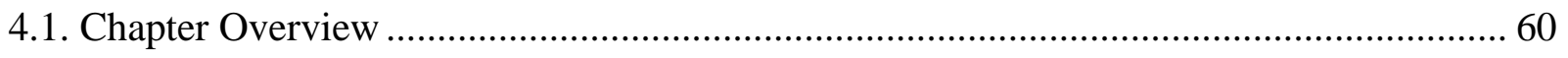




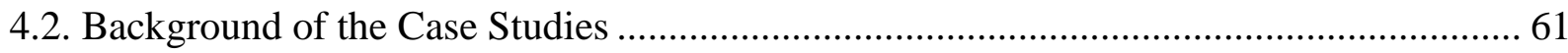

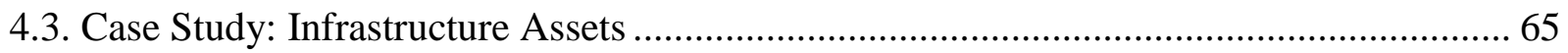

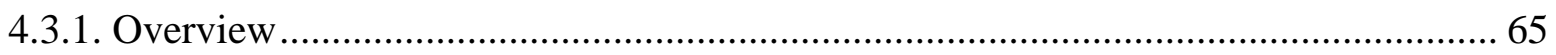

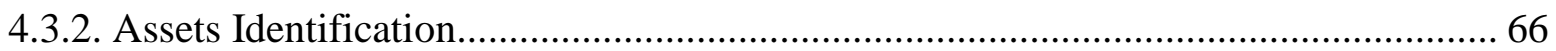

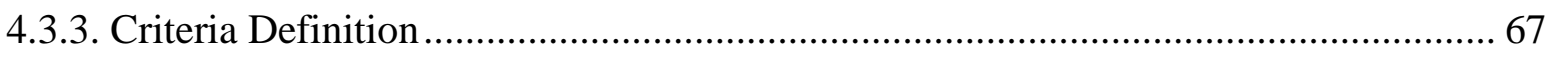

4.3.4. Baseline Scenario Multicriteria Analysis.................................................................. 72

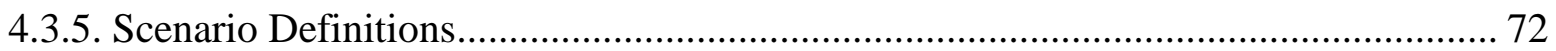

4.3.6. Criteria Importance Adjustment for New Scenarios.................................................. 73

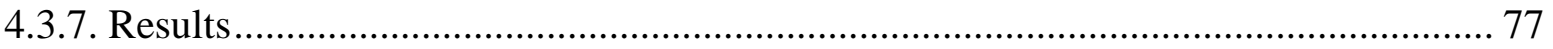

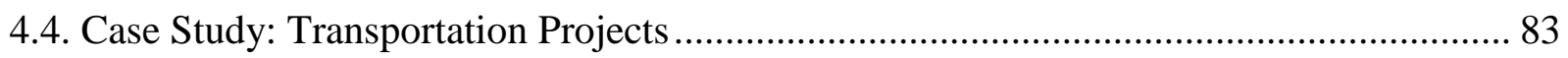

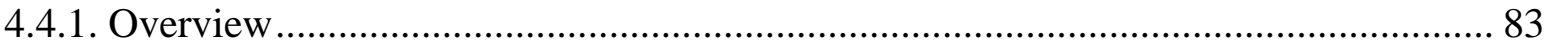

4.4.2. Identifying projects, criteria, and baseline value assessments ................................... 84

4.4.3. Defining and incorporating scenarios of deep uncertainties...................................... 90

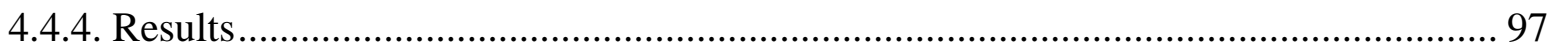

4.5. Case Study: VTrans 2035 Multimodal Policies............................................................. 104

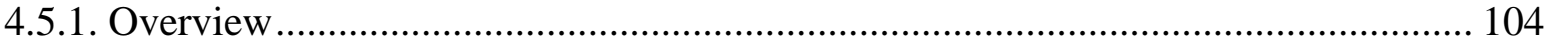

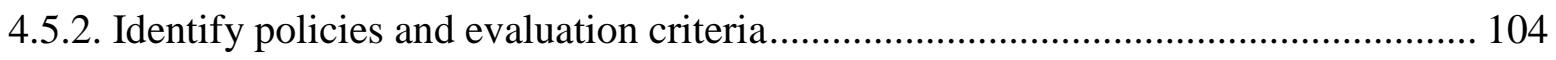

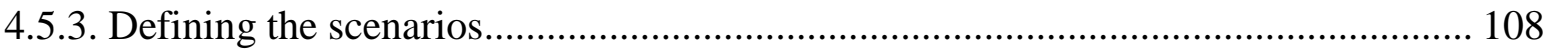

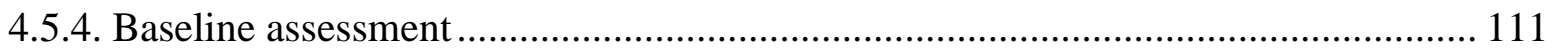

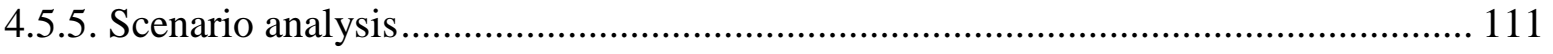

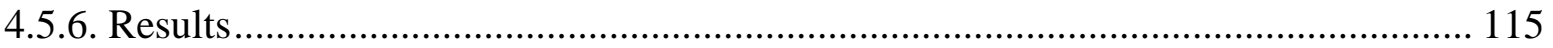

4.6. Case Study: Vulnerability Assessment for Traffic Analysis Zones................................ 121

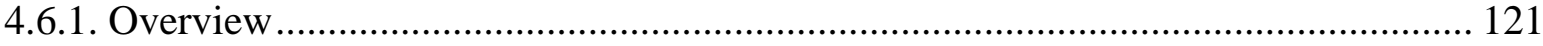




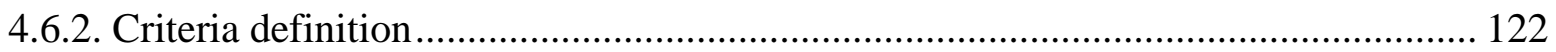

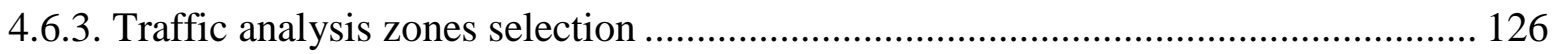

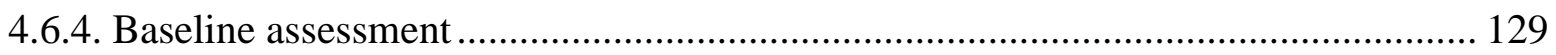

4.6.5. Scenario analysis and results ............................................................................ 136

4.7. Integrated Analysis across Multiple Case Studies ........................................................ 142

4.8. Lessons Learned from Case Studies ...................................................................... 143

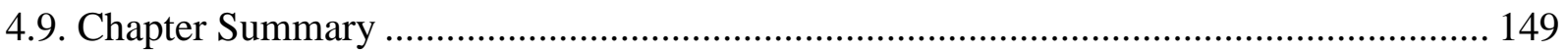

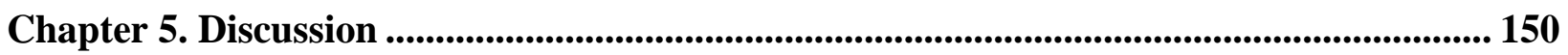

5.1. Chapter Overview ……………………………………………………………..... 150

5.2. Validation of the Introduced Analytical Framework ..................................................... 151

5.3. Applications of the Analytical Framework ……………............................................ 154

5.3.1. The "escape fish" of scenario analysis........................................................................ 154

5.3.2. Issues related to technology transfer.................................................................... 156

5.3.3. Natural disasters such as hurricane Katrina and Sandy ........................................... 158

5.3.4. To facilitate the vulnerability assessment of large single infrastructures ................. 160

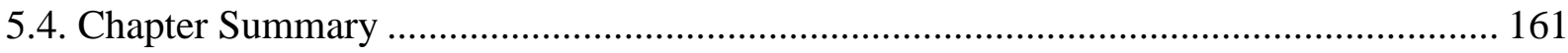

Chapter 6. Summary and Conclusions ................................................................................................. 162

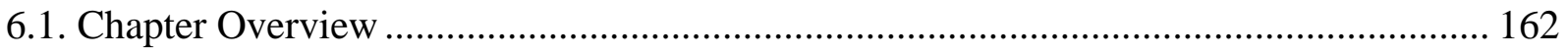

6.2. Review of Purpose and Scope................................................................................ 162

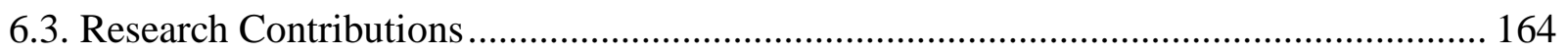

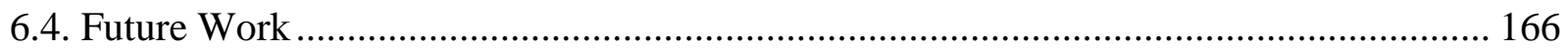

6.4.1. Quantitative and computerized scenario identification............................................ 166 
6.4.2. Survey-based analytical framework assessment.

6.4.3. Cost constrained analysis

6.4.4. Incomplete and imprecise information

6.5. Conclusion 170

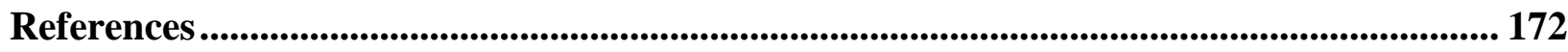




\section{List of Tables}

Table 4.2-1 Summary of projected climate change in the Hampton Roads region: 63

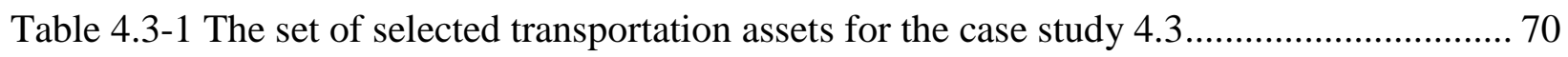

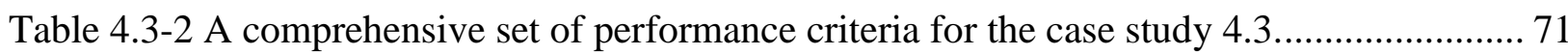

Table 4.3-3 The twelve selected assets (top row) are assessed on thirteen criteria (left column) in

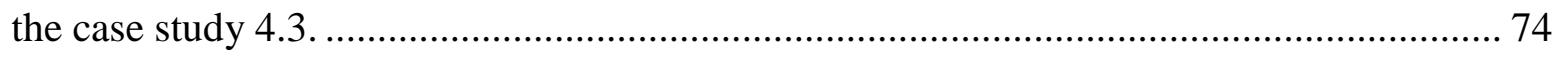

Table 4.3-4 The baseline scores and rankings of selected assets in the case study $4.3 . \ldots \ldots \ldots \ldots . . .75$

Table 4.3-5 Scenario definitions in the case study 4.3 ..................................................... 75

Table 4.3-6 Three scenarios (top row) and their shift in importance for the thirteen criteria (left

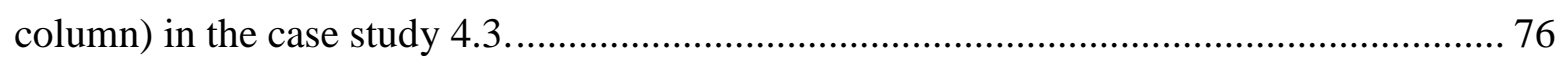

Table 4.3-7 The scores and rankings of selected assets across the baseline and additional three

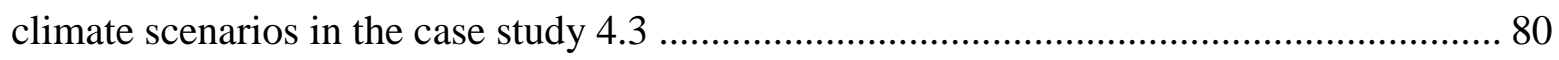

Table 4.4-1 A sample of the 93 candidate regional highway projects in the case study 4.4....... 86

Table 4.4-2 A comprehensive set of performance in the case study 4.4 .............................. 87

Table 4.4-3 A sample of the baseline value assessment of the 93 candidate transportation projects

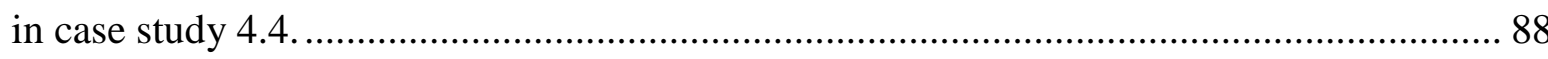

Table 4.4-4 The baseline scores and rankings of a sample of the 93 candidate regional highway

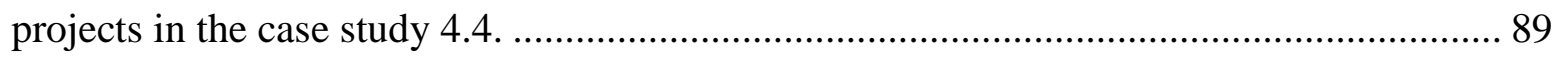

Table 4.4-5 The identified conditions and scenario definition in the case study $4.4 \ldots \ldots \ldots \ldots \ldots . . . . .94$

Table 4.4-6 The natural-language statements regarding the changes in relative importance for the

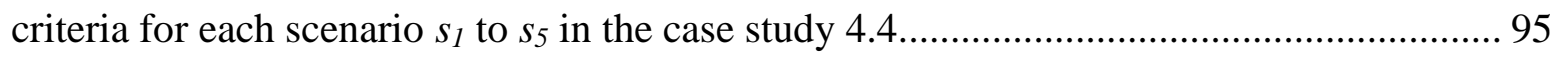

Table 4.4-7 The adjusted weights of the criteria for the scenarios $s_{1}$ to $s_{5}$ in the case study 4.4.. 96 
Table 4.4-8 The scores and rankings of a sample of the 93 candidate regional highway projects across five scenarios in the case study 4.4 . 100

Table 4.4-9 A characterization of the projects in terms of baseline priority ranking and changes in ranking induced by the scenarios in the case study 4.4 103

Table 4.4-10 The $\phi$ value quantifying the influence of scenarios, describing the influences of scenarios $s_{2}$ and $s_{5}$ are relatively greater than influence of other scenarios in the case study 4.4. 103

Table 4.5-1 The polices needing evaluation through scenario-based analysis in the case study 4.5 106

Table 4.5-2 Sample of the Policy Evaluation Criteria in the case study 4.5...... 107

Table 4.5-3 Emergent scenarios used for user reweighting of the criteria in the case study 4.5110

Table 4.5-4 Policy assessments against criteria in the case study 4.5. 113

Table 4.5-5 Criteria reweighting results from scenario-based methodology in the case study 4.5.

Table 4.5-6 Overall policy impact scoring of the statewide transportation policies in the case study 4.5 . 118

Table 4.6-1 A sample of the 50 selected Traffic Analysis Zones in the case study 4.6 127

Table 4.6-2 The GIS based baseline assessment for the 50 selected TAZs in the case study 4.6.

Table 4.6-3 Definition of conditions and scenarios constructed in the case study 4.6. 138

Table 4.6-4 Criteria relative importance adjustment for new scenarios in the case study 4.6... 139 Table 4.6-5 The disruptiveness of scenarios for the priority of TAZs in the case study $4.6 \ldots . . .139$ 
Table 4.8-1 The $\phi$ values and rankings of the influence associated to five scenarios from four

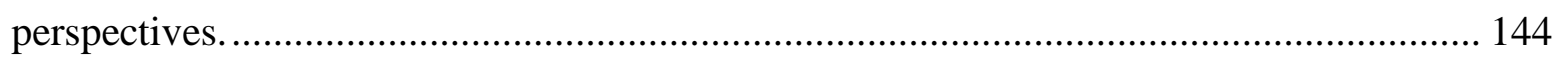

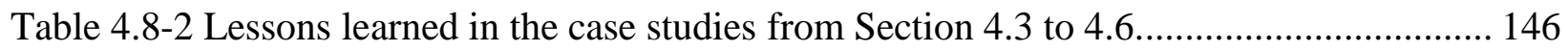

Table 5.4-1 The evaluation of the introduced analytical framework as a decision process ....... 153

Table 6.3-1 The research contributions of this dissertation.................................................. 165 


\section{List of Figures}

Figure 2.4-1 The scenario axis methodology for scenario analysis (Kahane and Senge, 2007) .. 21

Figure 3.6-1 Flow chart of the analytical framework introduced in this dissertation................ 47

Figure 4.2-1 A map of the region of Hampton Roads, Virginia .............................................. 64

Figure 4.3-1 The selection criteria for the list of infrastructure assets in the case study $4.3 \ldots \ldots . .68$

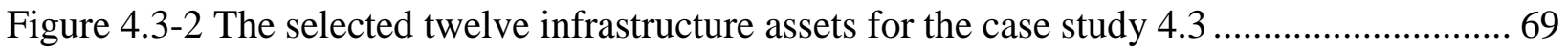

Figure 4.3-3 The baseline scores (diamonds) and the ranges (vertical bars) of scores associated to the three additional climate scenarios of the twelve infrastructure assets addressed in the

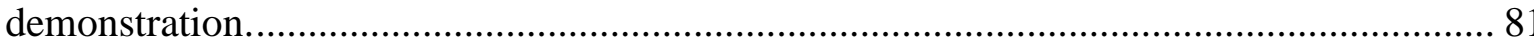

Figure 4.3-4 The baseline rankings (diamonds) and the ranges (vertical bars) of rankings associated to the three additional climate scenarios of the twelve infrastructure assets addressed in the demonstration

Figure 4.4-1 Frequencies of conditions being mentioned in science study assessment reviewed in the case study 4.4: (a) climate conditions (b) non-climate conditions. 93

Figure 4.4-2 The baseline scores (diamonds) and the ranges (vertical bars) of scores associated to the five scenarios of the 93 candidate regional highway projects addressed in the case study 4.4 101

Figure 4.4-3 The baseline rankings (diamonds) and the ranges (vertical bars) of scores associated to the five scenarios of the 93 candidate regional highway projects addressed in the case study 4.4 102

Figure 4.5-1 Range of policy impact scores with as-planned scenario policy score represented by diamonds in the case study 4.5 119 
Figure 4.5-2 Rankings of policies with sensitivities to five scenarios represented by the height of

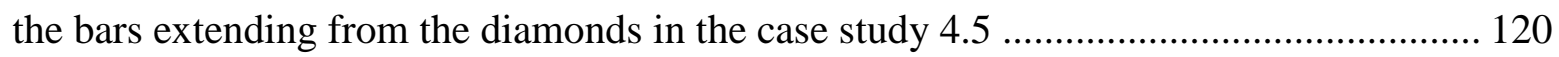

Figure 4.6-1 The overview map for the sampled $50 \mathrm{TAZs}$ in the case study $4.6 \ldots \ldots \ldots \ldots \ldots \ldots . . . . . .128$

Figure 4.6-2: Vulnerability map based on sea level rise in the case study $4.6 \ldots \ldots \ldots \ldots \ldots \ldots \ldots \ldots . . . . . . . . . .132$

Figure 4.6-3: Vulnerability map based on hurricane intensity in the case study $4.6 \ldots \ldots \ldots \ldots \ldots . . . . .132$

Figure 4.6-4: Vulnerability map based on road transportation in the case study 4.6 .............. 133

Figure 4.6-5: Vulnerability map based on multimodal facility in the case study $4.6 \ldots \ldots \ldots \ldots \ldots . . .133$

Figure 4.6-6: Vulnerability map based on population density in the case study $4.6 \ldots \ldots \ldots \ldots \ldots . . . .134$

Figure 4.6-7: Aggregated vulnerability map based on all criteria in the case study 4.6........... 134

Figure 4.6-8 The overall risk scores of all 50 selected TAZs across the future scenarios in the case study 4.6 140

Figure 4.6-9 The overall ranking of all 50 selected TAZs across the future scenarios in the case study 4.6 . 141

Figure 4.8-1 Demonstration of integrated framework with the influences of five scenarios to priority setting across four system perspectives: Projects, Assets, Policies, and Geographic Zones (TAZs). 145

Figure 4.8-2 Time horizons of different plans maintained by regional transportation planning agencies. 148

Figure 5.3-1 The website providing training and education materials for the implementation and transferring of the introduced framework 157

Figure 6.4-1 A sample of the decision process effectiveness questionnaire (Schilling et al., 2007) 168 


\section{Acronyms}

$\begin{array}{ll}\text { DOT } & \text { US Department of Transportation } \\ \text { EPA } & \text { US Environmental Protection Agency } \\ \text { FHWA } & \text { Federal Highway Administration } \\ \text { GIS } & \text { Geographic Information System } \\ \text { HHM } & \text { Hierarchical Holographic Modeling } \\ \text { HRPDC } & \text { Hampton Roads Planning District Commission } \\ \text { HRTPO } & \text { Hampton Roads Transportation Planning Organization } \\ \text { MCDA } & \text { Multicriteria decision analysis } \\ \text { TPO } & \text { Transportation Planning Organization } \\ \text { MPO } & \text { Metropolitan Planning Organization } \\ \text { NJDOT } & \text { New Jersey Department of Transportation } \\ & \end{array}$




\section{Notation}

$X=\left\{x_{1}, x_{2}, \ldots, x_{n}\right\}$

$Z=\left\{z_{1}, z_{2}, \ldots, z_{m}\right\}$

$\mathbf{A}=\left\{a_{j i} \mid i=1, \ldots, n ; j=1, \ldots, m\right\}$

$v: X \rightarrow \mathbb{R}$

$C=\left\{c_{1}, \ldots, c_{k}\right\}$

$P(C)$

$S_{c}=\left\{s_{0}, s_{1}, \ldots, s_{Q}\right\}$

$\hat{\mathbf{v}}_{x_{i}}=\left[v_{s_{0}}\left(x_{1}\right), v_{s_{1}}\left(x_{2}\right), \ldots, v_{s_{Q}}\left(x_{n}\right)\right]^{T}$

$\delta, \varepsilon$

$k \quad\left\{\begin{array}{ccc}k & k \\ 1 & 2 & 2 \\ 2 & , \ldots, & k \\ m\end{array}\right\}$

$\phi\left(s_{k}\right)$

$\tau_{j}^{k}$

$\left\{P_{P^{k}}\left(S_{i}\right)\right\}$

$\mathbf{P}=\left\{P^{1}, P^{2}, \ldots, P^{K}\right\}$
The set of decision alternatives

The set of decision alternative evaluation criteria

The matrix of evaluation results of decision alternatives

The multiattribute value function defining the order on the set $X$

The set of emergent and future conditions

The power set of the condition set $C$

The set of explorative scenarios

The multi-scenario profile of decision alternative $x_{i}$

The multipliers for the criteria weight adjustment

The set of possible natural-language statements about the importance change of a criterion comparing to the baseline scenario

The scenario influence quantified by decision alternatives

priority changes

The ranking of decision alternatives $x_{j}$ under scenario $s_{k}$

The multi-perspective vulnerability profile of system

The set of perspectives from which system vulnerability are concerned 


\section{Chapter 1. Introduction}

\subsection{Chapter Overview}

This chapter will introduce the topic of this dissertation. The sections of this chapter are organized as follows: Section 1.2 will describe the motivation of this study; and Section 1.3 will describe the organization of this document.

\subsection{Motivation}

Structural uncertainties, including climate, ecology, markets, regulations, social systems etc., which are influential to man-made systems, are now described as deep uncertainties. Deep uncertainty suggests situations in which the parties to a decision lack consensus on 1) the structural models to describe interactions among a system's variables, 2) the stochastic models to 
represent uncertainty about key parameters in the models, and 3) the methodologies to value the desirability of alternative outcomes (Lampert, 2007). Deep uncertainties need to be addressed for strategic decision makers and planners to make robust decisions for organizations and regional infrastructure systems.

Over the past two decades, climate change has been manifesting itself in a variety of ways. Evidences have showed that man-made infrastructure systems could be sensitive to and unpredictably affected by such changes (Brissette et al., 2006; Knogge et al., 2004; Desantis et al., 2007; Ackerman, 2008). Thus the potential impacts of climate change become one of the most critical concerns for planners and policy makers. However, due to the limited scientific understanding on the impact of climate change, the research on mitigation, rather than adaptation, of climate change drew much more public attention at the early stage of responding to climate change (Klein et al, 2004). U.N. Framework Convention on Climate Change (UNFCCC) and Kyoto Protocol demonstrated the prosperity of high level efforts to reduce anthropogenic greenhouse gas (GHG) emission in 1990s. Meanwhile, quantitative global climate models such as IPCC AR4 models and GCM hosted by NASA/GISS were developed to enforce better understanding of current and projection of future climate change. In light of results and conclusion from climate models, the US DOT, US EPA and state agencies compiled and announced their action plans respectively to reduce GHG emissions.

However even though these mitigation efforts are taking effect, the manifestations of climate change are expected to continue for decades or even centuries due to the inertia of the climate system (Tanaji and Vinod, 2007). Therefore in the most recent decades, a great portion of public attention turned to the issue of how to improve the adaptation of current man-made systems to the inevitable yet uncertain consequences of climate change. According to Tanaji and 
Vinod (2007) and Downing et al. (2005), effective adaptation to climate change needs to be integrated with current planning efforts, and is contingent on the availability of information on 1) what to adapt (identify the vulnerable sub-systems), 2) what to adapt to (identify most influential stressors) and 3) how to adapt (identify actions). Scenario analysis informed assets criticality prioritization and vulnerability assessment, which is able to help identify vulnerable resources and most influential stressors (Fussel and Klein, 2005), has been widely employed to answer the first two questions by agencies in various areas.

In the past decade, transportation agencies have begun to perform scenario-informed analysis in the strategic planning process at local and regional levels (McFarlane and Walberg, 2010; Larsen et al., 2007; Kirshen et al., 2006; Andrey and Knapper, 2003; Allen Consulting Group, 2005; Ibarrarán et al., 2008). Recently, several metropolitan planning organization pilots evaluate the vulnerabilities of infrastructure such as bridges and tunnels under the threats of storm surge from hurricanes exacerbated by sea-level rise (HRTPO, 2012; Lambert et al., 2012a; NJTPA, 2011; WSDOT, 2011).

These efforts indicate four trends on practices of vulnerability assessment:

1. The integration of consideration of climate change impacts to the existing strategic planning process;

2. The integration of analysis on climate change with the considerations of non-climate environmental and social-economic conditions (e.g. economic development, quality of life, and technologies); 
3. The integration of multiple "perspectives"1 on the vulnerable system;

4. The decrease of spatial scale on which the vulnerability assessments are conducted, which means limited budget for the analysis.

These trends expose the contradiction between two competing objectives in the context of vulnerability assessment, i.e. the limited investigative resources of regional or local agencies and the growing demand for comprehensive and integrated analysis. Therefore, a method of identifying the deep uncertainties that could most influence the strategic decisions with moderate resources is needed, in order that investigative resources could be focused to study the early warning, mitigation and adaptation of so-called deep uncertainties.

\subsection{Organization of the Dissertation}

This dissertation describes and tests an analytical framework to address these issues in strategic planning for transportation systems. The framework will significantly extend the previous efforts (Karvetski et al., 2009, 2011; Lambert et al., 2012; You et al., 2012) by:

- Identifying scenarios that combine climate with other sources of deep uncertainty including load/demand, economic, wear-and-tear, and ecological/environmental;

\footnotetext{
${ }^{1}$ In this dissertation, the term "perspective" refers to the different viewpoints to perform system analysis and system design, and to evaluate the overall performance of a system. For example, for a regional transportation system, the perspectives may include: emphasizing the significance of the improvements by transportation projects, focus on the operation and maintenance of transportation infrastructures, or others.
} 
- Changing the problem definition from seeking an optimal engineering alternative to priority-setting among candidate projects of a strategic portfolio, which might consist of hundreds of thousands of elements;

- Quantifying the influence of selected scenarios through a quantitative analysis and identifying the scenarios that most (and least) influence the priorities across projects;

- Assessing a system from multiple perspectives, and unifies the results from these angles and look for more holistic implications on the overall vulnerability of a system.

The remainder of this dissertation is organized as follows.

Chapter 2 reviews the literature related to climate change impacts, transportation planning process, scenario analysis, multicriteria decision analysis, and the integration of scenario and multicriteria methodologies.

Chapter 3 describes the analytical framework to incorporate multi-perspective scenario analysis on climate change to the current strategic transportation planning process, to obtain the assessment and risk profiles of individual decision alternatives and the whole system. Also the framework will be able to prioritize the scenarios in terms of their impacts on the system status, so that the further investigative, modeling and early-warning resources can be focused.

Chapter 4 demonstrates this framework with four case studies on the climate-change related system risk assessment for a coastal region of the United States. The demonstrations will focus on the data collection, expert knowledge elicitation, the analytical model and the integration of results. At the end of this chapter, an integrated analysis to consolidate the assessment results from multiple perspectives will be discussed. 
Chapter 5 discusses several topics related with the introduced analytical framework and the case studies.

Chapter 6 provides a summary of the dissertation, describes its contributions to the strategic planning in the context of emergent deep uncertainties such as climate change, and suggests opportunities for future effort. 


\section{Chapter 2. Background}

\subsection{Chapter Overview}

This chapter will review the relevant literature. The sections of this chapter are organized as follows: Section 2.2 will describe literature related to climate change and its impacts on transportation; Section 2.3 will describe literature related to the transportation planning process and methodology in the United States; Section 2.4 will describe literature related to scenario analysis and scenario planning; Section 2.5 and Section 2.6 will describe literature related to multicriteria decision analysis (MCDA) and an integration of MCDA with scenario analysis. 


\subsection{Climate Change and Impacts on Transportation}

Climate change is manifesting itself in a variety of ways. Over the last century, global sea level has risen 10 to 25 centimeters. With respect to the future climate change, many uncertainties exist and the existing climate models can be controversial. However, these models are the current state-of-the-art. Although it is possible climate change will not take place as predicted, from the perspective of risk analysis, it is rational to assume that the general direction of climate change that projected by main stream global climate models is correct. The future sealevel rise induced by climate change ranges from 20 to 86 centimeters for the year 2100 , with a best estimate of 49 centimeters (Pachauri and Reisinger, 2008). Rising sea level increases coastal erosion and amplifies storm and flooding damage, which pose threats to infrastructure systems (USGCRP, 2009). On the other hand, the average global temperature has been projected to rise up to $6^{\circ} \mathrm{C}$ by the year 2100 (Pachauri and Reisinger, 2008). Some regions are expected to experience up to 90 additional days in which heat index ${ }^{2}$ is more than $100^{\circ}$ (US EPA, 2009). The temperature rise can further intensify regional contrasts in precipitation, which results in more frequent and severe floods and droughts in many regions.

Among the above, sea level rise and the increase in frequency and intensity of storm surges and flooding may be the most worrying consequences of climate change, especially for coastal areas. The IPCC 2007 report mentioned that for North America, coastal flooding due to sea level rise and storm surge is one of the most destructive impacts of climate change. The damage will be especially strong along the Gulf and Atlantic coasts (Field et al., 2007). Gornitz

${ }^{2}$ The heat index (HI) is an index that combines air temperature and relative humidity in an attempt to determine the human-perceived equivalent temperature. For example, when the temperature is $32^{\circ} \mathrm{C}$ with very high humidity, the heat index can be about $41^{\circ} \mathrm{C}$. 
(2001) even predict that infrastructures in some coastal areas along the Gulf of Mexico and the Atlantic will be permanently inundated sometime within 100 years.

The Intergovernmental Panel on Climate Change (Pachauri and Reisinger, 2008) and the Stern (2007) analyzed the damages of climate change on agriculture, health and insurance and other sectors. The consequences of climate change for the transportation section has not received much attention, even after 2010, when FHWA and local planning organizations started to fund some studies on this topic. However, it is widely believed that transportation system will perform worse under more frequent / longer duration of extreme weather conditions.

Jacob et al. (2001) pointed out that many elements of the transportation system in the US Metropolitan East Coast region lie less than 6 meters above the sea level in 2001. They showed that low elevations of important infrastructures in metropolitan areas, for example New York City, are at risk of being flooded more intensely while the sea-level keeps going up (Jacob et al., 2007).

A report from ICF International (ICF, 2008) has analyzed the impact of sea-level rise on the transportation infrastructure at the East Coast of US. The report pointed out that if no protective actions are taken, some transportation infrastructures will be periodically inundated due to the seal-level rise and storm surge. Although the infrastructures will be impacted is a small portion, the inundations are expected to lead to large degradation on the transportation system due to network effect.

The Gulf Coast region has been the object of several studies (Kafalenos and Lenonard, 2008). They found that in a $61 \mathrm{~cm}$ sea-level rise scenario, 64 percent of the port facilities will be impacted. And the $122 \mathrm{~cm}$ sea-level rise scenario will affect up to 75 percent of the port 
facilities. The proportion of highways and rails are considerably low, but the impacts are still substantial. Moreover, the report also pointed out the potential large issues will be brought by the network effect even the impact is not widely spreaded. As for the storm surge, the study indicated that up to 60 percent of the roads, 40 percent of the railway, and 30 percent of the airports are vulnerable to surges of 5.5 to 7 meters.

Longer duration of high temperatures brought by climate change may cause the pavement of roadways to soften and expand, and subsequently place additional stress on bridge joints. High temperature can also influence the efficiency of construction activities, especially in areas with high humidity. Therefore, it could become much more costly to maintain roads and highways (USGCRP, 2009). Exposure to flooding and extreme cold may shorten the life expectancy of transportation infrastructures. Road infrastructure in coastal areas is particularly vulnerable to more flooding from sea level rise and storm surges. Currently, about 60,000 miles of coastal roads in the United States are at risk of coastal flooding (US FHWA, 2008). In some locations, rising temperatures are believed to cause more winter flooding. Landslides and wash-outs may occur more frequently as well, if saturated soils are exposed to more rainwater. On the other hand, drought in areas may increase the likelihood of wildfires that threaten roads and other infrastructure. Climate change may also affect the traffic safety and travel times, thus the coastal region may suffer from the loss on the network and mobility and the associated economic loss. 


\subsection{Transportation Planning under Deep Uncertainties}

\subsubsection{The metropolitan planning organization (MPO)}

According to the document by the study of Johnston (2004), the transportation planning in the United States generally follows a three-phase pattern: pre-analysis, technical analysis, and post analysis. During this process, the metropolitan planning organization (MPO) plays an essential role.

In the United States, a MPO is a federally mandated and federally funded transportation policy-making organization ${ }^{3}$. MPO is made up of representatives from local government and governmental transportation authorities (Bond et al, 2010), and is required for any urbanized area (UZA) with a population greater than 50,000 according to the Federal-Aid Highway Act of 1962. Federal funding for transportation projects are channeled through the planning process of MPO, based on a continuing, cooperative, and comprehensive ("3-C") planning process (US FHWA, 2007). Public involvement and participation in the planning process of MPO is required by federal law now.

The missions of a MPO include (Bond et al, 2010):

1. Establish a setting: establish and manage a fair and impartial setting for effective regional decision-making in the metropolitan area;

${ }^{3}$ As of 2012, there are 342 MPOs in the United States. 
2. Evaluate alternatives: evaluate transportation alternatives, scaled to the size and complexity of the region, to the nature of its transportation issues, and to the realistically available options;

3. Maintain a long-range transportation plan (LRTP): develop and update a fiscally constrained long-range transportation plan for the metropolitan area covering a planning horizon of at least twenty years that fosters mobility and access for people and goods, efficient system performance and preservation, and quality of life;

4. Develop a transportation improvement program (TIP): develop a fiscally constrained program based on the long-range transportation plan and designed to serve the metropolitan area's goals while using spending, regulating, operating, management, and financial tools

5. Involve the public: involve the general public and all the significantly affected subgroups in the four essential functions listed above.

\subsubsection{Transportation planning to address climate change}

Mitigation of climate change drew much public attention at the early stage of responding to climate change (Klein et al., 2004). U.N. Framework Convention on Climate Change (UNFCCC) and Kyoto Protocol demonstrated high level efforts to reduce anthropogenic greenhouse gas (GHG) emission in 1990s. Meanwhile, quantitative global climate models such as IPCC AR4 models and GCM hosted by NASA/GISS were developed to enforce better understanding of current and projection of future climate change. In light of results and conclusion from climate models, the US DOT, US EPA and state agencies compiled and 
announced their action plans respectively to reduce GHG emissions. However even these mitigation efforts are taking effect (Tanaji et al., 2007), the manifestations of climate change are expected to continue for decades or even centuries due to the inertia of the climate system. Therefore currently, a great portion of public attention turns to the issue that how to improve the adaptation of current man-made systems to the inevitable yet uncertain consequences of climate change. According to the Fussel and Klein (2005), effective adaptation to climate change is contingent on the availability of information on 1) what to adapt, 2) what to adapt to and 3) how to adapt. Vulnerability assessment, which examines the system to identify vulnerable resources and most influential stressors (Klein et al., 2007), has been widely employed to answer the first two questions by agencies in various areas.

Agencies such as FHWA and FTA took great initiatives to address climate change.

American Association of State Highway and Transportation Officials (AASHTO) published a white paper examines how current transportation asset management (TAM) can integrate the extreme weather risk brought by climate change into the decision making and strategic planning process of transportation agencies (AASHTO, 2012). The paper reviews the risk assessment methodology for transportation assets and explained why and how extreme weather is different from other risk. It also introduced how consideration of extreme weather can be integrated from the beginning to the final steps of the TAM process with the help of data collection and management.

FHWA initialized a few programs and published a few white paper / guidebooks to direct the integration of climate change adaption into the transportation planning and assets management. A conceptual Risk Assessment Model was issued during the pilot project of "Assessing Vulnerability and Risk of Climate Change Effects on Transportation Infrastructure" 
in 2010. The model was piloted in five State Departments of Transportation MPOs. The goal of the model was to help transportation planners to identify critical assets exposed to the threats from climate change. FHWA refined the original conceptual model and develop a final version for all transportation agencies with the lessons learned during the pilot phase. FHWA has posted a final, updated framework in 2013. The updated framework provides an overview of key steps to perform vulnerability assessments. In-practice examples are attached to demonstrate the methodologies to gather and process information (US FHWA, 2011).

In 2011, the FHWA published a report on the application of geographic information system to support the transportation planning under the uncertainty of climate change. The report synthesized the lessons learned from eight case studies at state, regional and local level and gave recommended practice on the development and application of GIS tools to support analysis of climate change mitigation and adaptation strategies.

Another agency, the Federal Transit Administration (FTA), published a report on public transportation and climate change adaptation in 2011 (US FTA, 2011). The report provided an overview of the anticipated climate impacts on the public transit systems of the United States. It also reviewed the on-going climate change adaptation efforts by transit agencies in the world; the covered topics include risk management, vulnerability assessment, and application of adaptation planning tools. The report discussed how transit agencies should incorporate climate change adaptation planning and policy implementations into their organizational structures, asset management system and operation processes. FTA is also maintaining a web page named "FTA Climate Change Adaptation Initiative". The page includes the resources including scientific research reports, summary of pilot projects, materials from recent workshops and webinars, and documents about FTA and DOT policies on climate change. 


\subsection{Scenario Analysis}

\subsubsection{A historical view}

Scenario analysis can trace its history back to the eighteenth century. In the work "On War", Carl von Clausewitz, the Prussian military theorist explored the differences of military objectives under different political situations. He presented several scenarios to illustrate how the army should react in a right manner. However, these scenarios do not have some essential features of modern scenarios. For example, these scenarios were visionary instead of being systematically developed. Furthermore, there was no storyline for how these scenarios emerged from the current situation (Bassford, 1994).

A modern approach to scenario analysis emerges right after the Second World War. In 1950s, RAND Corporation introduced a new technique called "future-now thinking" to promote debate about nuclear weapons (Ringland, 1998). This technique aimed to produce archive reports for current events (as they might be analyzed retrospectively by people living in the future) and create stories about future. This technique is usually considered as the prototype of modern scenario analysis, despite the term scenario analysis was not used that time. In 1968, another organization that pioneered in scenario analysis, Stanford Research Institute (SRI), undertook the task of creating future scenarios for the US Department of Education (Ringland, 1998). SRI developed five scenarios. One of them assumed that the present trends would continue (the surprise-free scenario) and the remaining four scenarios were the possible answers to two questions: 1) whether the society would be able to control its destiny and 2) whether the U.S. society would be open or authoritarian. The method used by SRI in this project is considered a precursor or prototype of the modern "two-ax" scenario construction method. 
From the 1970s, scenario analysis started to be adopted by private sectors. Royal Dutch/Shell was one of the companies that were well known for its planning system evolution. In early 1960s, the planning horizon of Shell's planning system was five years and the deliverables are projected numbers and figures (Wack, 1985). In 1967, Pierre Wack outlined the need for long-term planning and introduced a technique named "scenario planning." By using this technique analyzing the predetermined and uncertainties of the global business environment, Shell's management was prepared for the oil crisis in 1973 and 1981. When other oil companies stockpiled reserves in the aftermath of the outbreak of the Iran-Iraq war, Shell sold off its excess before the glut came and prices collapsed. After the oil embargo, Shell managed to become the one of the strongest multinational oil companies while before it was among the weakest. Inspired by the success of Shell, the companies affected by the oil crisis (mainly in the oil industry and the automobile industry) started to realize the value of scenario analysis in anticipating surprises and discontinuities. Scenario analysis thus drew attentions and started to be widely used. This approach has continued to the present day.

The use of scenario techniques was dramatically increased in the 1980s for a number of reasons. The main reason for that was that the oil crisis was becoming the "lessons to learn". As mentioned above, the crisis re-shaped the global business thus many companies incorporate scenario analysis into the existing planning system. Furthermore, a recession, which led to the significant losses by many large companies, such as General Electric, Xerox and Chrysler, took place in the beginning of 1980 s. The fact that this discontinuity was not predicted enlarged the mistrust of the available forecast methodologies. The literature indicates some efforts for creating new methodologies for planning. Michael Porter, for example, accepts scenarios as a tool and recommends them as a form of sensitivity analysis. He invented one of the most 
important of scenario analysis principles - internal consistency (Ringland, 1998). In these decades, a number of consulting companies had emerged to specialize in scenario analysis services using a variety of methodologies.

From the 1990s, the business world started to emphasize the importance of "strategy", in which scenario analysis plays a central role. There was a trend towards smaller teams to take the responsibility for planning projects as well as the broader range of external uncertainties to be considered in scenario analysis. Practitioners also have been seeking the possibility to merge quantitative methods such as probability theory and its variants to the framework of scenario analysis. In addition to that, the application areas of scenario analysis were extended to public sectors. To address uncertainties in natural and socio-economic systems, public agencies and non-profit organizations began to employ scenario analysis in their long-term planning processes (McFarlane and Walberg, 2010; Larsen et al., 2007; Kirshen et al., 2006; Andrey and Knapper, 2003; Allen Consulting Group, 2005; Ibarrarán et al., 2008).

\subsubsection{Empirical studies}

Scenario analysis is addressed extensively by empirical studies. Numerous studies report the application of scenario techniques. As the most well-known case study, Wach (1985a, b) summarized how Shell developed the planning system which managed to anticipate the uncertain futures. Peterson et al. (2003) summarized some cases including Shell's strategic planning, a national wide business, political and civil of South Africa, the future of ecosystem service in the Northern Highland Lake District, Wisconsin. The paper argues that in some gradual changed situations, hedging, adaptive management, and optimal management may be appropriate. But 
when control is difficult and uncertainty is high, scenario analysis is proved to be an effective method to cope with the future challenges. Zegras et al. (2004) introduced a framework for scenario-planning techniques for regional strategic transportation-planning purposes. This framework induced an eight-step scenario planning approach, which was successful to facilitate the long-range planning of the Houston metropolitan area. Ogilvy and Smith (2004) summarized the utilization of scenario analysis, which serve the public interest, such as the projects for the US EPA, for the California Energy Commission and for the city of Austin, Texas. The studies concluded that these projects "met with varying degrees of success". Parson et al., (2007) comparing four scenario exercises related to global change applications suggests that climate scenarios are useful to support further modeling and analysis, and at the same time they can also help frame public debates. Several studies report that scenarios can have positive outcomes in terms of individual learning. Using scenarios can increase participants' perceptions of their strategic communication and conversation skills, as a recent study that gathered data on individual participants in a scenario-planning project demonstrated (see Chermack et al., 2006). Another example can be found in California, where at a series of workshops managers and stakeholders of a California water agency were presented with decision aids incorporating scenario concepts. An increasing understanding of the challenges posed by climate change and their shifting views on how best to respond were noted during the exercise (Groves et al., 2008). These studies are often cited as examples of the benefits of developing and using scenarios.

Harries (2003) argues that most of these case studies focus on isolated and unique cases and therefore the reported successful results are not convincing enough. Phelps et al., (2007) compares the performance of organizations that do and do not use scenario-based planning. Though among water companies, scenario planning produced mixed results. Among IT 
companies, scenario planning seems correlated with higher profit and return on investment. Clark et al., (2006) perform similar comparison analysis in the area of environmental consulting, and found adoption scenario analysis generally correlated to better strategic decision support.

Some studies seek the factors that allow scenarios to be used successfully. In the articles introducing the evolution of the planning system of Shell, Wach (1985a, b) emphasized the value of Shell's two-stage scenario method: use first-generation exploratory scenarios to understand the environment, and then assemble decision scenarios with both uncertainties and predetermined elements to help the strategic decision making. Cornelius et al. (2005) provides some detailed insights on Shell's scenario analysis from a methodology perspective. Hodgkinson and Wright (2002) argue that the culture of the organization is very important to the success of scenario techniques. Managers may reject scenarios that identify threats for which there are no viable responses. Much literature emphasizes the need for consensus on the relevant variables for scenarios in order to foster a common basis of understanding (van 't Klooster and van Asselt, 2006).

\subsubsection{Best practices and methodologies}

With large amount of empirical studies indicating the cognitive and methodological advantages of scenario analysis, literatures started to summarize the best practice and document methodologies. It is widely agreed that most of the benefits of scenario analysis manifest during the scenario construction. Moreover, from a process standpoint, scenario construction is a prerequisite of subsequent communication, analysis and decision-making. Therefore, scenario construction is justifiably considered as the critical step in the process of scenario analysis. 
Extensive literatures have review the methods emerge in practice and introduced new methodologies for scenario construction.

Chermack (2001) summarized three overarching categories of early scenario building and development approaches: 1) Kahn's qualitative approach based on reasoned judgment and intuition (Kahn and Weiner, 1967), 2) The quantitative approach favored by some management scientists. For example, Amara and Lipinski (1983) propose an approach that they augmented by operational research and management science (OR/MS); Mavromatis and Jones (1998) use a random number generator to stochastically generate climate scenarios based on long-term historical data. Computer assistance has largely involved in this category of approaches, 3) the middle ground between the two approaches above, such as described by Millet and Randle (1986) procedural scenarios generation, which incorporate both intuitive and quantitative techniques.

Bishop et al. (2006) take an historical perspective, tracing three main schools: the intuitive logics school, which originated with RAND and is now strongly associated with Shell Oil and the Global Business Network; the La Prospective school developed in France by Gaston Berger and Michel Godet; and the Probabilistic Modified Trends school originally developed by Ted Gordon and Olaf Helmer at RAND. Bishop et al. conclude that most scenario practitioners use the scenario axis approach (as described by Figure 2.4-1) associated with Shell Oil and the Global Business Network. In that approach, a set of key driving forces is identified first and the two driving forces regarded to be most important and most uncertain in terms of their future development form the axes of a matrix. They determine the overall logic of the scenario storylines (Bishop et al., 2006). This intuitive school is consistent with the qualitative category described in Chermack's review. Pierre Wack from Shell applied Kahn's concepts in the 1960s and refined them into a new framework. Peter Schwartz took over Wach's framework as the head 


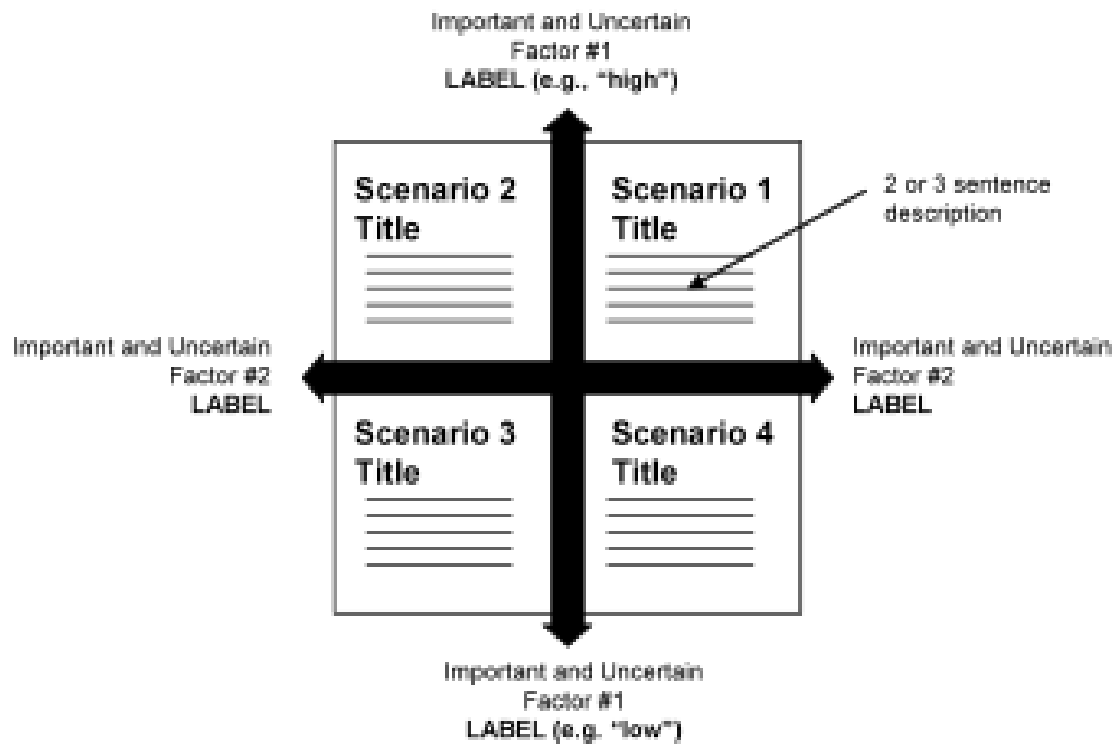

Figure 2.4-1 The scenario axis methodology for scenario analysis (Kahane and Senge, 2007) 
of Shell's planning division and eventually established the Global Business Network. Schwartz (1991) presented the famous "two-axes" scenario building process in his work, The Art of the Long View. This book forms the basis of the approach used by the Global Business Network.

Bishop et al. (2007) review the construction methodology from a functional perspective. This paper identifies eight categories of scenario development techniques, namely judgment; trend extrapolation; elaboration of fixed scenarios (incasting); event sequences (probability trees, sociovision, divergence mapping); backcasting; dimensions of uncertainty (scenario matrix, morphological analysis); cross-impact analysis; and modeling. It reviewed all the techniques and summarized their utility, strengths and weaknesses. The paper emphasizes that most scenario practitioners have latched on to the Shell/GBN scenario matrix approach.

Aside from the classic methodologies covered by these reviews, some literatures independently introduced innovative methods. O'Brien (2001) introduces a particular scenario development methodology advocated in the 1970s and 1980s, which for a number of years has formed a core component of a strategic development course taught at the University of Warwick. O'Brien identifies a number of common pitfalls concerning scenario development and recommends a revised methodology that addresses them. On the basis of several case studies, van Notten et al. (2003) propose suitable methods to create different categories of scenarios in his typology, which differentiates scenarios according to their goal (either raising awareness or decision support). Hua-dong (2003) formulates the objective of picking scenarios as to minimize the loss of information (increase of entropy). Based on this problem definition, the paper proposes a series of mathematical principles to complement the many existing informal techniques of scenario analysis: plausible futures should be all equally valid, the bundle $\mathrm{S}$ should be plausible, and it should describe a wide range of possible futures. Groves and Lempert, (2007) 
introduced a computer-assisted scenario discovery method based on assumption-based planning (Dewar, 2002) and robust decision-making methods (Lempert et al., 2003). The method uses statistical cluster-finding algorithms to help users identify the combinations of input parameters of the environment models, so that the scenarios are strongly predictive of those results most important to the strategic decisions.

\subsubsection{Theoretical research}

While the practices of scenario analysis are flourishing, the relevant literature reveals a gap regarding its theoretical foundation. Theoretical research for scenario analysis is expected to answer fundamental questions such as "what is a scenario", "why scenario analysis is able to help", "what is the future from the perspective of scenario analysis" and "what are the criteria for good scenarios", to potentially overcome the non-repeatable and the subjective characteristics of scenario analysis that have been reported in empirical studies.

Practitioners have defined scenario analysis in many ways. Michael Porter (1985) defined scenarios as "an internally consistent view of what the future might turn out to be-not a forecast, but one possible future outcome". Schwartz (1991) defined scenarios as "a tool for ordering one's perceptions about alternative future environments in which one's decisions might be played out". Ringland (1998) defined scenario analysis as "that part of strategic planning which relates to the tools and technologies for managing the uncertainties of the future". Schoemaker (1995) suggested the definition "a disciplined methodology for imagining possible futures in which organizational decisions may be played out". These definitions provide some perspectives to understand scenarios and scenario analysis, but are not described in a precise and scientific way. 
Some scholars have sought to formalize the essential of scenarios and explain the theoretical benefit of scenarios. Schoemaker (1993) defines scenarios as focused descriptions of fundamentally different futures presented in coherent script-like or narrative fashion. The paper described the behavioral foundations of scenarios by the results of four psychology experiments. Scenarios seem to operate using psychological effects to help expand people's frames, overcome the availability bias, where people undervalue that which is hard to imagine or recall from memory, and shift the anchor or baseline from which people view the future. The paper also argues that presenting scenarios as possibilities, rather than as firm predictions, may enhance their psychological impact because they become less threatening to those holding different worldviews. This is consistent with Hua-Duong's argument on intentional ignorance (2007). Hua-Duong (2003) tried to formulate the scenario analysis with the theory of imprecise probability. He models the scenario analysis as a situation of one-way communication with limited bandwidth to transmit the detailed knowledge. Chermack (2005a) define scenario analysis as a method to boost strategic conversation. His argument is built on a experiment conducted on individual participants in a scenario planning project. Data concerning perceptions of strategic conversation skills were collected before and after the scenario planning project. The descriptive statistics indicate an overall increase in mean scores from pre- to post- intervention assessment. This finding captures the effect of scenario planning on individual perceptions of communication skills. While publishing this experiment based research, Chermack (2005b) offer a theory framework of scenario planning based on Dubin's eight-step quantitative theory building research method. The framework has specified multiple hypotheses and suggestions for studying scenario analysis. Taxonomy is another perspective for theoretical research. Borjeson et al. (2006) propose a three-part taxonomy that differentiates the questions scenarios address about the future. 
Predictive scenarios ask: What will happen? Explorative scenarios ask: What can happen? Normative scenarios ask: How can a specific target be reached?

Climate scenarios exist that address each of these questions. As an example, Borjeson et al. categorize both projections from atmospheric ocean general circulation models (AOGCMs) and greenhouse-gas emission scenarios like SRES as explorative. Other perspectives include Garb (2008)'s definition on dimension of social process. He defines scenarios as social products and social processes, based on the fact that scenarios could reflect and 'fix' certain framings and assumptions implicitly. Lempert (2007) summarizes a number of theoretical researches efforts and points out that currently for those aiming to supply information about future conditions to decision-makers, the term "scenarios" could mean: 1) standardize cases used for planning, modeling and communicating; 2) detailed narratives describing some paths into the future; 3 ) highly uncertain forecasts.

Lempert argues that such diversity might be valuable because users can choose the way to use scenarios according to the project requirements. However, it still reflects a lack of a clear answer to the fundamental questions mentioned at the beginning of this section. The long lasting debate on whether probabilities should be assigned to scenarios manifests itself as one aspect of this confusion.

\subsubsection{In the context of risk analysis}

Kaplan and Garrick (1981) introduced the set of triplets as the definition of risk. Scenario $s_{i}$ is the first tuple in the triplet $\left\langle s_{i}, p_{i}, x_{i}\right\rangle$, and it answers the first one of the three questions for risk analysis: what can happen (i.e. what can go wrong). For each scenario, a probability $p_{i}$ and 
a consequence $x_{i}$ are assigned to describe how likely it that the scenario will happen is, and if it does happen, what will be the consequences. Risk analysis is subsequently defined as identifying the set of risk triplets that are relevant to the problem to be investigated.

In the same year, Haimes (1981) introduced a structural view of risk scenarios - the Hierarchical Holographic Modeling (HHM). The essential of the HHM is that for complex system, the risk modeling and analysis can be holistic when multiple different perspectives are taken into consideration. The generality of the HHM drew the attention of the society of risk analysis to the fact that different methodologies of scenario identification / structuring can lead to different scenario partitioning results and subsequently different risk analysis results. This fact somehow challenged the "set of triplets" definition of risk.

In order to eliminate this awkwardness of the "set of triplets" definition of risk, Kaplan et al. (2001) refined the original triplet definition of the risk. The refined definition admitted that the set of risk scenario set in practice is highly dependent on the scenario structuring methodologies, and thus removed the specific set of scenario from the original "set of triplets" definition of risk and casts it as an approximated partition to the true set of scenarios that is "native" to the problem. The refined definition allowed that the set of risk scenarios to be nondenumerable. And this continuous set of scenarios constitutes the "true" set of risk. In practical problems, the true scenario set may be partitioned into a series of subset. And the HHM can serves as a way of partitioning the true set of risk scenarios. Another important argument in this paper is that it is usually not practical to perform quantitative risk analysis on all scenarios. Therefore, risk filtering is necessary to systematically filter down the scenarios to a manageable set that can be concentration of further modeling and investigative resources. 
Florig et al. (2001) argued that ranking risks may be more effective as the initial activity than ranking the action alternatives, and the ranking of risks can be a very critical input for the decision making. An effective risk ranking procedure should include decision analysis, risk analysis, behavioral theories and other methodologies. At the same time all possible sources of information should be involved to enhance the understanding the risks to be ranked. The procedure should also highlight the agrees and disagrees on the ranking of risks from different stakeholders and participants. Morgan et al., 2000; Morgan et al., 2001 pointed out that the risk ranking system can be constructed using a multi-criteria model, that is similar to multiattribute value function.

Haimes et al. (2002; 2009a) introduced the Risk Filtering, Ranking, and Management (RFRM) framework. The framework utilized methodologies including the HHM, qualitative consequence and likelihood comparisons, and other multicriteria and probabilistic approaches to filter and set ranking to a large set of scenarios. With this framework, the identified risk scenarios / partitions will be filtered or prioritized systematically, according to the value of risk manager, stakeholders, or the decision maker.

\subsection{Multicriteria Decision Analysis}

\subsubsection{Multiattribute utility theory and multiattribute value theory}

Multiattribute utility theory (MAUT) is the multiattibute extension of the unidimensional utility theory, which was established by von Neumann and Morgenstern's Theory of Games and Economic Behavior (1947). And multiattribute value theory (MAVT), which was initialized by 
the work of Luce and Tukey (1964), Kranz (1964), Luce (1966), and Tversky (1967), is the certainty version of the MAUT from the perspective of value measurement.

According to von Winterfeldt and Edwards (1986), value is typically a transformation on some physical scale, and utility is a further transformation on value, intended to take into account the decision maker's attitude toward risk. The idea of MAVT is to construct a method of associating a real number with each alternative, so that a complete order of preference on these alternatives can be obtained. The method must assure that the preference order is consistent with the value judgment of the decision makers. In other words, MAVT seek a way to assigning a real number $v(a)$ to each alternative $a$, so that a is preferred to a' $\left(a \succ a^{\prime}\right)$ if and only if $V(a)>V\left(a^{\prime}\right)$, and a is indifference with $\mathrm{b}\left(a \sim a^{\prime}\right)$ if and only $V(a)=V\left(a^{\prime}\right)$.

MAVT achieved this goal with a two steps process: 1) model the value preference on single attribute by marginal (partial) value functions $v_{i}(a) ; 2$ ) apply an aggregation model to combine single attribute values: $V(a)=f\left[v_{1}(a), \ldots, v_{n}(a)\right]$. The aggregation can be modeled by an additive function if all attributes are "preferential independent" (Keeney and Raiffa, 1993).

MAUT extended the MAVT using the concept of probabilities and expectations to deal with uncertainty. MAUT assume for each alternative, each of its attribute value $X_{i}(a)$ is not fully determined by the choice of alternatives, but also be influenced by some random factors (Belton and Stewart, 2002). These random factors can be described by a probability distribution on the attribute values. MAUT seeks a function $U(a)$, such that a is preferred to a' $\left(a \succ a^{\prime}\right)$ if and only if $E[U(a)]>E\left[U\left(a^{\prime}\right)\right]$, and a is indifference with $\mathrm{b}\left(a \sim a^{\prime}\right)$ if and only $E[U(a)]=E\left[U\left(a^{\prime}\right)\right]$. This condition is the expected utility hypothesis (Keeney and Raiffa, 1993). 
Obviously, any function $U(a)$ is also a proper value function for MAVT. However, a value function $V(a)$ does not necessarily satisfy the requirements of MAUT. Comparing MAVT, MAUT also requires more strict assumptions. Attributes need to be utility independent and additive independent to enable an additive form of $U(a)$.

\subsubsection{Multicriteria decision analysis in systems analysis and risk analysis}

In the theory of systems engineering (Sage and Armstrong, 2000), the early phases of the system lifecycle include the problem definition, the construction of high-level objectives and a value model and, the identification of alternatives, the evaluation of alternatives within the developed value framework, and finally the refinement of alternatives. Buede (2000) pointed out that in the preliminary or strategic phases of system development, stakeholder contributions are the key inputs. Multicriteria decision analysis can provide guidance to the stakeholders involvement with respect to the value framework (Kleinmuntz, 2007; Keeney, 1992).

The multicriteria decision analysis is inherently able to handle multiple competing objectives of complex problems such as decisions on long-term investment in infrastructure, and a lot of economic and environmental decisions. Literatures from different areas (Linkov et al., 2008; Linkov et al., 2007; Linkov et al., 2006a; Keeney and McDaniels, 2001) proved that multicriteria decision analysis is effective to support decision-making in areas of public policyanalysis related to deep uncertainty such as climate change. Chatzimouratidis and Pilavachi, 2009a, 2009b; Nigim et al., 2004 have utilized the multicriteria decision analysis methodologies

in the selection of energy alternatives. As one of the multicriteria decision analysis method, the 
ELECTRE III method was used for ranking renewable energy alternatives on insular Greek islands (Papadopoulos and Karagiannidis, 2006).

Loken (2007) evaluated different multicriteria models such as value measurement models, reference level models, and outranking models in the context of building energy networks with a single energy carrier. This research argued that for large-scale integrated energy systems, these multicriteria models need to be able to deal with multiple stakeholders or decision makers with conflicting objectives. And Kowalski et al. (2009) introduced the applications of "participatory" multiple criteria decision analysis to address complexity for evaluating renewable energy sources in Austria. Challenges including describing complex scenarios in both narrative and quantitative way and inconsistent stakeholder participation are raised as essential problems in the application.

\subsection{Integration of Multicriteria Decision Analysis and Scenario Analysis}

In the last decades, multicriteria decision analysis and scenario analysis are integrated as the tool to support long-term and strategic planning. The earliest difficulty of the integration is that it is very hard and even misleading to determine the quantitative probabilities of the scenarios. Stewart (2005) argued that the identified scenarios may not be complete and the estimation can only relay on subjective judgment and thus differ across different stakeholders. Some behavior studies (Goodwin and Wright, 2001; Tversky and Kahneman, 1974) support this argument by experiments indicating faulty heuristics and overconfidence. Another difficulty in front of this integration is that traditional multicriteria value theory is based on one deterministic future (Comes et al., 2009). 
Several case studies and applications have undertaken to link scenario-based analysis with multicriteria decision analysis. These applications usually define a limited set of scenarios (three or even fewer) (Comes et al., 2009; Montibeller et al., 2006; Goodwin and Wright, 2001). An additive value function is frequently used, but other approaches have also been utilized, for example goal programming (Durbach and Stewart, 2003).

Goodwin and Wright (2001) first use multiattribute value theory as a formal decision analysis approach in traditional scenario analysis. Though multiattribute value theory cannot be applied to situations with uncertainties, value judgments under certainty are used to get overall evaluation of the alternatives on the occurrence of a specific scenario. In this paper, Goodwin and Wright evaluate each alternative using a single additive multiattribute value function. All alternatives are evaluated and assigned a value score for each criterion. The additive multiattribute value function aggregates these scores to give each alternative an overall value score under a particular scenario. These value scores are used to prioritize the overall performance and robustness of each alternative across the scenarios.

Montibeller et al. (2006) used multiattribute value theory approach to deal with alternative ranking in epistemic uncertainty. The paper observed that the parameters of additive value function, which represents the value tradeoffs and preferences, are different through different scenarios. Thus Montibeller et al. (2006) create a scenario-dependent additive value function for each scenario to evaluate the alternatives across the scenarios. The obvious disadvantage of Montibeller et al.'s work is that the preference and value model elicitation needs to be repeated for each scenario. The time and resources used on the elicitation is a multiplier of the elicitation time, let alone the behavioral bias caused by repetitive interviews and communications. An example is that in the case study of Ram et al. (2010), which incorporate 
twelve diverse scenarios to evaluate the robustness of food security options in Trinidad and Tobago. The elicitation process is replicated for each scenario. The paper pointed out that the repetition and the resource utilization are the most frequently mentioned complaints of the interview process. The application of the integration between multicriteria decision analysis and scenario analysis is acknowledged as partially problematic due to the significant elicitation burden.

Karvestki et al. (2009) introduced a scenario-based multicriteria decision analysis framework to elicit non-structured knowledge from local experts and consolidate the knowledge with a multiattribute value theory, so that prioritization assessments can be both budget-friendly and informative for regional and local agencies. The framework was used to prioritize infrastructure projects in a border province of Afghanistan with modest investigative resources.

More recent case studies and applications of the integration of scenario analysis and multicriteria decision analysis include statewide multimodal transportation planning (Schroeder and Lambert, 2011), the identification of most influential climate change scenarios (Karvetski et al., 2011a, 2011c), and the priority-setting of risk-communication strategies for the siting of liquefied natural gas terminals in Mexico (Martinez et al., 2011; 2010).

\subsection{Chapter Summary}

This chapter has established a foundation for the analytical framework, case studies and discussions in the coming chapters by review the relevant literature. Section 2.1 provided a chapter overview; Section 2.2 described literature related to climate change and its impacts on transportation; Section 2.3 described literature related to the transportation planning process and 
methodology in the United States; Section 2.4 described literature related to scenario analysis and scenario planning; Section 2.5 and Section 2.6 described literature related to multicriteria decision analysis (MCDA) and the integration of MCDA with scenario analysis. 


\section{Chapter 3. Analytical Framework}

\subsection{Chapter Overview}

This chapter will describe the analytical framework of this dissertation. Section 3.2 will first describe the multicriteria decision analysis model to set the baseline (no scenario) priority for a group of decision alternatives for a planning effort; Section 3.3 and 3.4 will explain how to elicit non-structured knowledge to assess the impacts of external stressors incrementally based on the baseline results; Section 3.5 will discuss the metrics which can be used to aggregate the priority changes of all decision alternatives when considering a future scenario. These metrics will be used to quantify the impacts of these future scenarios to the system; Section 3.6 will extend the scenario influence quantifying method to support analysis from multiple perspectives; At the end of this chapter, Section 3.7 will discuss the MCDA model selection and the elicitation of input variable for the proposed analytical framework. 


\subsection{Multicriteria Priority-Setting on Decision Alternatives}

In long-term and strategic transportation planning, decision alternatives compete for human, financial and policy resources. Setting priority for these alternatives is among the essential deliverables of a planning effort. In this section, we will formulate the general structure of a single perspective priority-setting process.

When evaluating a system from a single perspective, priority-setting is built on a 3-tuple $\{X, Z, \mathbf{A}\}$, where $X=\left\{x_{1}, x_{2}, \ldots, x_{n}\right\}$ is the set of decision alternatives to be prioritized; $Z=\left\{z_{1}, z_{2}, \ldots, z_{m}\right\}$ is the set of criteria for evaluating and prioritizing the alternatives; $\mathbf{A}=\left\{a_{j i} \mid i=1, \ldots, n ; j=1, \ldots, m\right\}$ is a matrix, which represents the assessment of the alternatives $X$ across the criteria set $Z$. In the priority-setting process, each alternative $x_{i} \in X$ is evaluated under each criterion $z_{i} \in Z$ to produce a score or value assessment $a_{j i} \in \mathbf{A}$, where $a_{j i} \in[0,1]$. The assessment is usually elicited from local experts or calculated based on data and models from technical reports in terms of how $x_{i}$ address the objectives measured by $z_{j}$. The relation $a_{j p}>a_{j q}$ implies that alternative $x_{p}$ addresses the objective of criterion $z_{j}$ more sufficiently than $x_{q}$.

To prioritize the alternatives is to set a complete order (i.e., all alternatives are comparable as more, less, or equal to one another) on the set of alternatives $X$, with the multicriteria assessments A. Thus we define a multiattribute value function (Keeney, 2012) $v: X \rightarrow \mathbb{R}$ and define the order on the set $X$ as that $x_{i}$ is more preferred than $x_{j}$, denoted as 
$x_{i} \succ x_{j}$, if and only if $v\left(x_{i}\right)>v\left(x_{j}\right) ; x_{i}$ and $x_{j}$ are equally preferred, denoted as $x_{i} \sim x_{j}$, if and only if $v\left(x_{i}\right)=v\left(x_{j}\right)$

Keeney and Raiffa (1993) and Belton and Stewart (2002) discussed different forms of multiattribute value functions and described how to construct these functions in practice. Within these forms, the additive form is most prevalent. Additive value function requires independence assumptions to be examined on the criteria set $Z$. In the context of transportation planning and vulnerability assessment, the mutual independence condition among these assumptions usually cannot be fully satisfied. However, Stewart (1996) proved that the additive value function is generally robust to prioritize alternatives with mild violations of this assumption. Therefore, for illustration purpose, we consider an additive value function given by

$$
v\left(x_{i}\right)=100 \times \sum_{j=1}^{m} w_{j} a_{i j}
$$

where $\left\{w_{j} \mid \sum_{j=1}^{m} w_{j}=1,0 \leq w_{j} \leq 1, j=1, \ldots, m\right\}$ is the normalized weights for the criteria.

The above method only supports baseline system assessments, which prioritize decision alternatives based on a normal system evolution assumption. It is not sufficient to evaluate a system without consideration of threats of uncertain future stressors. We will extend the method to address uncertainties by introducing scenario analysis in the following section.

\subsection{Priority-Setting Informed by Scenario Analysis}

Ideally, priority setting for decision alternatives in strategic transportation planning should rely on precise science and engineering models of natural and social systems. However, 
due to the complexity of natural and human eco-social systems, most models are high level and only able to giving insights on a global or continental scale. Regional planning agencies thus could hardly utilize the low-resolution data and conclusions in these high-level models. Agencies then have to either collaborate with regional institutes to re-model the local natural and ecosocial system, or project the magnitude and frequency of stressors from historical data. These efforts usually require intensive investigative resources, which are very limited for regional agencies. The process may also be time-consuming and fruitless because a large portion of stakeholder participation will focus on reaching an agreement on the credibility of these results from models and projects. Nevertheless, the results may be misleading, especially when stressors correlate with and exaggerate each other.

Rather than predicting the future and modeling the system, the objective of strategic transportation planning is to identify the optimal and robust resource (funding and investigative) allocation for the system. Therefore, scenario analysis is proper to be used to consider possible emergent conditions and condition combinations in future. In scenario analysis, a scenario is a plausible future, which described by a group of environmental and socio-economic parameters. Decision alternative assessment and prioritization can be performed across a set of scenarios to understand the interaction between future scenarios and the transportation system, and estimate the impact of potential risks on the consequences (not likelihood) dimension. On the other hand, scenario analysis enforces the perception of a scenario by narrative statements and visual materials. It thus takes fully advantage of implicit and non-structured knowledge of local experts and planners to make value judgments and impact assessments.

According to Bishop and al et. (2007), majority of scenario creating methods follow the structure of identifying critical driving forces first and then assembling these forces into 
scenarios. Here we give a simplified formulation of scenarios accordingly. We define a set of emergent and future conditions $C=\left\{c_{1}, \ldots, c_{k}\right\}$, which will later be combined to create scenarios. The identification of these conditions is various by case studies. Each element of the power set of the conditions set $C$, denoted as $P(C)$, is a combination of the conditions. Any elements of $P(C)$ that are inherently consistent and meaningful are candidates of scenarios for further analysis. Thus the set of scenarios $S_{c}=\left\{s_{0}, s_{1}, \ldots, s_{Q}\right\}$ is subset of $P(C)$, i.e. $S_{c} \subseteq P(C)$. For convenience, we named the non-condition case as the baseline scenario, denoted as $S_{0}$

\subsection{Incremental Adjustment of Value Functions for Scenarios}

The baseline value function eq. (1) evaluates decision alternatives in the baseline scenario only, representing implicit/unstated assumptions. In this section, scenarios constructed to bound possible stressors are considered to account for the influences to the baseline value function. We will thus be able to quantify and compare the influences of scenarios in terms of their disruptiveness to the baseline priority-setting result.

As described in the previous section, a set of preliminary scenarios $S_{C}$ is constructed.

Each scenario $s_{k} \in S_{C}$ will influence the priority-setting result derived by baseline value function $u_{0}$ in the following way. We define value functions for each scenario $s_{k}, v_{s_{k}}: X \rightarrow \mathbb{R}$. For each alternative $a_{i}$, each scenario $s_{k} \in S_{C}$ will thus be able to inspire a new evaluation. The evaluations of each alternative $x_{i}$ under $S_{c}$ can be described by a vector $\hat{\mathbf{v}}_{x_{i}}=\left[v_{s_{0}}\left(x_{1}\right), v_{s_{1}}\left(x_{2}\right), \ldots, v_{s_{Q}}\left(x_{n}\right)\right]^{T}$. This vector $\hat{\mathbf{v}}_{x_{i}}$ is called the multi-scenario profile of the 
decision alternative $a_{i}$. Similar with the baseline case, a complete order is defined by the relation of $v_{s_{k}}\left(a_{i}\right)>v_{s_{k}}\left(a_{j}\right)$ on the set of alternatives $A$ for each scenario $s_{k}$.

The influences of scenarios on the value function are modeled by a swing-weight technique based incremental value elicitation method (Karvestski, Lambert and Linkov, 2009). The value function for scenario $u_{s_{k}}$ is thus defined as an incremented version of $u_{s_{0}}$,

$$
v_{s_{k}}\left(x_{i}, \Gamma^{k}\right)=\sum_{j=1}^{m} w_{j}^{k} a_{i j}^{0}
$$

where $\Gamma^{k}$ is the expert opinions elicited about the change of public value in the context of scenario $s_{k}$. For each scenario $s_{k},{ }^{k}\left\{\begin{array}{c}k \\ 1\end{array},{ }_{2}^{k}, \ldots,{ }_{m}^{k}\right\}$ is a set of possible natural-language statements $\eta_{j}^{k}$ about the importance change of a criterion comparing to the baseline scenario $s_{0}$. For the purpose of demonstration, we define $\eta_{j}^{k}$ to be one of five statements: major increase, minor increase, no change, minor decrease, or major decrease. Each statement $\eta_{j}^{k}$ will affect the criteria weights according to the following rules,

$$
w_{j}^{k} \propto\left\{\begin{array}{ll}
w_{j}^{0}(\delta) & \text { if } \eta_{j}^{k}=\text { "major increase" } \\
w_{j}^{0}(\varepsilon) & \text { if } \eta_{j}^{k}=\text { "minor increase" } \\
w_{j}^{0} & \text { if } \eta_{j}^{k}=\text { "no change" } \\
w_{j}^{0}(1 / \varepsilon) & \text { if } \eta_{j}^{k}=\text { "minor decrease" } \\
w_{j}^{0}(1 / \delta) & \text { if } \eta_{j}^{k}=\text { "major decrease" }
\end{array}\right\}
$$

such that $\sum_{j=1}^{m} w_{j}^{k}=1$ and $0 \leq w_{j}^{k} \leq 1 . \delta, \varepsilon(\delta>\varepsilon)$ are multipliers that reflect the magnitude of value change under additional scenarios. They may be interpreted as comparative or relative worth multiplier. Given a certain scenario, the practical interpretation of $\delta$ and $\varepsilon$ is that the 
worth of exchanging the alternatives is $\delta$ and $\varepsilon$ times than that under the baseline scenario (Karvetski et al. 2009). Sensitivity analysis and weights range sensitivity index (RSI, Nitzsch and Weber, 1993) check can aid in verifying if a group of given values are proper.

Note that Eq. (2) considers that $a_{i j}^{k}=a_{i j}^{0}$ for $k=1, \ldots, Q$. In our experience, this assumption is reasonable for a long time horizon in which the assessment $\left\{a_{i j}^{k} \mid 0 \leq a_{i j}^{k} \leq 1\right\}$ is

more permanent than the weights of criteria $\left\{w_{j}^{k} \mid j=1, \ldots, m\right\}$. In the cases such as long-range planning regarding infrastructures, calculations and judgments based on physical attributes are used in the assessment phase. These inputs do no significantly change across different scenarios. Rather, scenarios alter the value system of decision makers. We have found in most real-world cases, the decision makers are comfortable with this assumption. For other situations, the ratings could be re-assessed per scenario.

\subsection{System Vulnerability Assessment via Priority Setting Results}

As mentioned in the first chapter, other than setting the priority of decision alternatives across a set of possible future scenarios, performing vulnerability assessment to identify the most threatening deep uncertainties, and then providing decision aids on further investigative / precaution resource allocation is another objective of strategic transportation planning. From the perspective of priority-setting, we are also interested in finding which scenarios are most and least influential to the priorities of decision alternatives, so that scenarios can be kept or discarded from consideration based on their relative influences. This is especially important when the number of decision alternatives greatly exceeds the number of scenarios. 
In order to accomplish these objectives, a framework for quantitative measurement of the scenarios' impact to the priority-setting result on the ordinal scale, denoted as $\phi\left(s_{k}\right)$, is developed in this section. The framework is set up to assure the developed the measurement to be unbiased and informative. For single perspective planning efforts, the framework consists of two requirements:

1. With the value of $\phi\left(s_{k}\right)$ for each $s_{k} \in S_{c}$, we will be able to conclude that the system is more vulnerable to the stressors in $s_{i}$ than those in $s_{j}$, if and only $\phi\left(s_{i}\right)>\phi\left(s_{j}\right)$; if $\phi\left(s_{i}\right)=\phi\left(s_{j}\right)$, we may consider the system is equally vulnerable to the stressors described in both $s_{i}$ and $s_{j}$.

2. The measurement should only reflect those priority changes, which is influential to the planning results.

The first requirement assures values of the measurement reflect the magnitude of the impact if a future scenario happens to the system. The second requirement ensures that the value of summarizing the information which is important to decision-makers and planners. For example, if the objective of a priority-setting process is to select 5 most critical infrastructures to conduct protection and resilience program, a ranking change from 8th down to 12th for some infrastructure should not contribute to the value of a well-defined measurement.

Generally, the priority of all decision alternatives can be informative to decision makers at the early stages of the strategic transportation planning. Thus a typical measurement of scenario's influence can be defined as the sum of all decision alternatives' ranking changes 
against the rankings in the baseline scenario. Then we can easily define the measurement $\phi\left(s_{k}\right)$ for scenario $s_{k}$ as:

$$
\phi\left(s_{k}\right)=\sum_{i=1}^{n}\left|\tau_{i}^{k}-\tau_{i}^{0}\right|
$$

where $\tau_{j}^{k}$ is the ranking of decision alternative $x_{j}$ under scenario $s_{k}$.

It is trivial to prove that SRMR satisfy the both two requirements. And planners could easily find other measurements which satisfy the two requirements defined above. For instance, we may define another measurement $\tilde{\phi}\left(s_{k}\right)$, which is equivalent to $\tilde{\phi}\left(s_{k}\right)$ by substituting the operating of absolute value with square ${ }^{4}$.

$$
\tilde{\phi}\left(s_{k}\right)=\sum_{i=1}^{n}\left(\tau_{i}^{k}-\tau_{i}^{0}\right)^{2}
$$

where $\tau_{j}^{k}$ is the rank of decision alternative $x_{j}$ under scenario $s_{k}$.

Depending on the specific cases, the definition of the objectives of strategic transportation planning usually manifests itself as more analysis results and public inputs are obtained. In this case other measurements may need to be developed. For example, suppose the planners and stakeholders reach to the agreement to select most critical $N$ alternatives from the candidates via priority-setting, then an appropriate measurement may be:

$$
\hat{\phi}^{k}=\sum_{i=1}^{n} \frac{n_{i}^{k}}{N}
$$

${ }^{4}$ The variance of values of $\tilde{\phi}$ is greater than $\phi$, which may make $\tilde{\phi}$ not be preferred by planners. 
where $n^{k}$ is the amount of decision alternatives that are not ranked in the top $N$ decision alternatives in baseline scenario but rank among the top $N$ decision alternatives under scenario $s_{k}$. This measurement emphasizes the relevant information and eliminates noises. However, it no longer maintains all the information generated by the priority-setting process.

\subsection{Integrated Analysis from Multiple Perspectives}

As mentioned in the first chapter, besides involving localized expertise and being resource friendly, an emerging theme of strategic transportation planning is the integration of information from multiple perspectives. In this sense, the decision alternatives need to provide a holistic viewpoint that unifies different perspectives, to assure the decision-making for strategic transportation planning are made towards overall regional objectives rather than the interests of specific stakeholders.

In this section, we extend the single perspective methodology defined in section 3.1 through 3.4 and define a integrated multi-perspective vulnerability assessment as a 2-tuple $\left\{S_{c}, \mathbf{P}\right\}$, where $S_{c}$ is the set of future scenarios representing potential stressors to the system; $\mathbf{P}=\left\{P^{1}, P^{2}, \ldots, P^{K}\right\}$ is the set of different perspectives from which the strategic planning. Each perspective $P^{k} \quad \mathbf{P}$ has similar structure and is represented by a 3 -tuple $\left\{X^{k}, Z^{k}, \mathbf{A}^{k}\right\}$. In other words, within each individual perspective, we will replicate the process of identifying alternatives, setting priority, and quantify scenario impacts and evaluate the system vulnerability. When the process is replicated for each perspective $P^{k} \quad \mathbf{P}$, we will a multi-perspective vulnerability profile $\left\{{ }_{P^{k}}\left(s_{i}\right)\right\}$ fo the system, where $P^{k} \quad \mathbf{P}, k=1, \ldots, K$ and $s_{i} \quad S_{c}, k=1, \ldots,\left|S_{c}\right|$. 
And we will be able to select and discard some of the scenarios in $S_{c}$ to focus the investigative and modeling resources. This process is illustrated in Figure 3.1.

The method to interpret multi-perspective vulnerability assessment results various from case to case. In some cases, it is feasible to aggregate the multi-perspective vulnerability profile $\left\{{ }_{P^{k}}\left(s_{i}\right)\right\}$ across perspectives via ${ }_{\mathbf{P}}\left(s_{i}\right)=w_{P^{k} P^{k}}\left(s_{i}\right)$ by eliciting weights for the perspectives $w_{P^{k}}$ according to their relative importance. However, the elicitation process can be timeconsuming and the result can be misleading. Moreover, when the amount of considered perspectives is small, it is not necessary to aggregating the results at the cost of losing detailed information.

In order to make the value of measurement $\phi_{p^{k}}\left(S_{i}\right)$ comparable among the different perspectives, the definition should eliminate the impact of the number of decision alternatives of analysis on different perspectives. A possible definition is

$$
\phi\left(s_{k}\right)=\lambda \sum_{i=1}^{n}\left(\tau_{i}^{k}-\tau_{i}^{0}\right)^{2}
$$

where $\tau_{i}^{0}$ is the ranking of decision alternatives $x_{j}$ in the baseline scenario and $\tau_{i}^{k}$ is the ranking value under scenario $s_{k}$. The coefficient $\lambda$ is introduced to normalize the value of $\phi$, so that it is bounded in the interval $[0,1] . \lambda$ is given by the value of $\phi$ when the decision alternatives priority is fully inverted (i.e. $\tau_{i}^{k}=n+1-\tau_{i}^{0}, i=1 \ldots n$ ), because $\phi$ reaches its maximal value under this circumstance ${ }^{5}$.

\footnotetext{
${ }^{5}$ A sketched proof can be found in the addendum.
} 


$$
\lambda=\frac{3}{n\left(n^{2}-1\right)}
$$

The metric $\phi$ exaggerates large changes in rankings. A scenario that drops a highly prioritized decision alternative to being prioritized close to the last will typically have higher value of $\phi$ than a scenario that switches the ranks of a few decision alternatives which are near to one another in the priority order. A higher value of $\phi$ identifies a scenario that is more influential to the baseline priority-setting result.

However, the bounded metric can only work with full rankings (i.e., permutations). It will be biased in the situation where alternatives tie for some positions in the ranking. Unfortunately, ties are common when the priority-setting largely reply on categorical or natural-language based assessments. We introduced the metric called Kendall Tau-b rank distance (Laurencelle, 2009) to address this problem.

In the rank distance area, Gamma statistic and Kendall tau-b are usually used to measure the distance between two sets of ordinal values when ties are allowed (Simon et al. 1997). Gamma statistic simply ignores the tied paired, which tends to underestimate the disruptiveness of a scenario in our context. Kendall tau-b performs adjustments for ties based on original Kendall tau distance (Laurencelle, 2009). Moreover, the value Kendall tau coefficient is nearly normally distributed for small $\mathrm{n}$ and the distribution is easier to work with (Luke et al. 2001).

We thus built our metric based on the formulation of Kendall tau-b. Let $r_{i}^{0}$ is the ranking of decision alternative $a_{i}$ in the baseline scenario and $r_{i}^{k}$ is the ranking value under scenario $s_{k}$. $\left(r_{1}^{0}, r_{1}^{k}\right),\left(r_{2}^{0}, r_{2}^{k}\right), \ldots,\left(r_{n}^{0}, r_{n}^{k}\right)$ is a group of ranking values that influenced by scenario $s_{k}$. Any pair of ranking values $\left(r_{i}^{0}, r_{i}^{k}\right)$ and $\left(r_{j}^{0}, r_{j}^{k}\right)$ are said to be concordant if both $r_{i}^{0}>r_{i}^{k}$ and $r_{j}^{0}>r_{j}^{k}$ or 
both $r_{i}^{0}<r_{i}^{k}$ and $r_{j}^{0}<r_{j}^{k}$. They are said to be tied, if $r_{i}^{0}=r_{i}^{k}$ or $r_{j}^{0}=r_{j}^{k}$. Otherwise the pair $\left(r_{i}^{0}, r_{i}^{k}\right)$ and $\left(r_{j}^{0}, r_{j}^{k}\right)$ is discordant. The metric is thus given as,

$$
\phi\left(s_{k}\right)=\frac{1}{2}\left[1-\frac{n_{c}^{k}-n_{d}^{k}}{\sqrt{\left(n_{c}^{k}+n_{d}^{k}+t_{0}\right)\left(n_{c}^{k}+n_{d}^{k}+t_{0}\right)}}\right]
$$

where $n_{c}$ is the number of concordance rankings, $n_{b}$ is the number of discordance rankings, $t_{0}$ is the number of rankings tied in the priority-setting result under baseline scenario, and $t_{k}$ is the number of rankings tied in the priority-setting result under scenario $s_{k}$. 


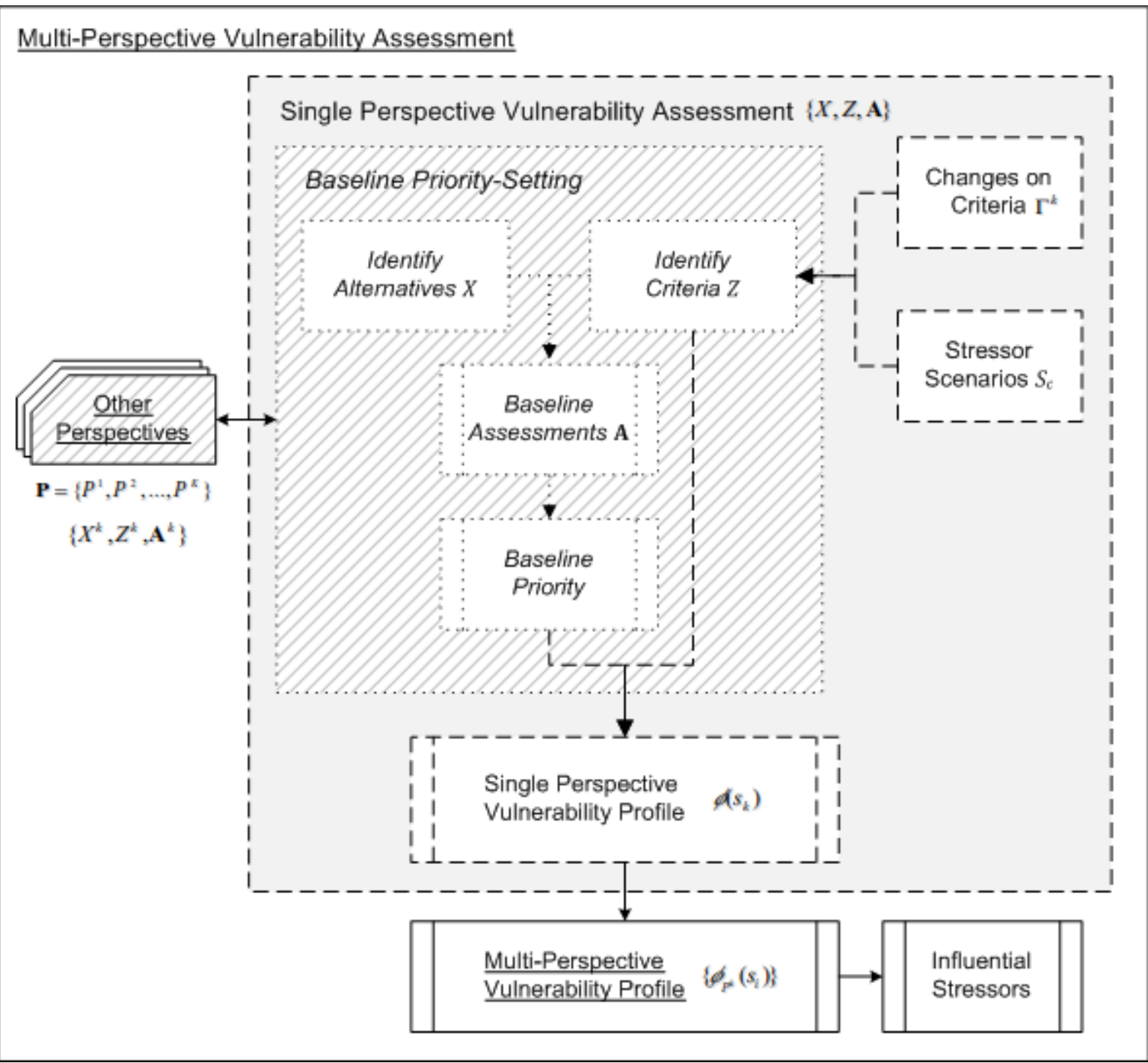

Figure 3.6-1 Flow chart of the analytical framework introduced in this dissertation 


\subsection{On the Implementation of the Analytical Framework}

\subsubsection{The Selection of Multicriteria Decision Analysis Methods}

As described in Section 3.2, multiattribute value theory (MAVT) based method is used in the research as the multicriteria decision analysis (MCDA) method. However in literatures, we can find more than thirty case study supported MCDA methods (Belton and Stewart, 2002; Figueira et al., 2005). Other than MAVT/MAUT methods, examples of MCDA methods include analytical hierarchy process (AHP), VIKOR method, ELECTRE, PROMETHEE and etc (Satty, 1980; Figueira et al., 2005). Moreover, after decades of advancement on research and applications, MCDA methods have appeared in an even more diffuse way, without any clear general methodology or basic theory (Vincke, 1992). In this section, we will discuss the selection of MCDA method.

As a prescriptive decision aiding tool, the MCDA method in this analytical framework mean to provide a structured way of modeling the decision problems in order to maximize the understandings of the problem, and to subsequently improve (not determine) the outcome of the decision process. As argued by Roy (1996), MCDA methods should not search for an optimal solution, but rather aim at helping decision makers to master the complex judgments and data involved in their problems and advance towards an acceptable solution. In other words, from being focused on analysis and selection in a set of decision alternatives, MCDA is expected to be more focused on providing models and tools to help decision-makers explore the problems and solution space. The output from these methods may not be interpreted as the solutions to the decision problems. Instead, the outputs provide a well-defined picture of the essentials of the decision problem and the potential consequences of certain actions. In this sense, a decision 
support method cannot just provide guidelines for the decision analytical calculations. The support for the decision making process and compatibility to the existing organization operational process, cultures and structures are among the critical considerations (French and $\mathrm{Xu}$, 2005).

The applications of the analytical framework are in the field of regional transportation planning. Weighted sum model (Fishburn, 1967) based multicriteria projects selection and assets prioritization systems are widely utilized in the existing planning process (see section 2.3). Tools, expertise and experience in working with criteria identification, trade-off based criteria weights development, and value satisfaction level based assessment can be accessed with moderate cost. Therefore comparing to other MCDA methods, the MAVT based method can be easily adopted by these organizations.

Another reason for choosing MAVT method as the default MCDA method in the analytical framework is the consideration to keep the process straightforward and easy to implement. Literature suggested most important for the practical applicability of MCDA methods is the easiness of the method implementation (Stewart, 1992; Hulle et al, 2011). Simpler tools are often easier to use and therefore more likely to be useful and less likely to produce errors (Edwards and Barron, 1994). Some even claim that simpler and frugal methods can produce results that are almost as good as results to those obtained by more complicated analysis (Katsikopoulos and Fasolo, 2006; Larichev et al., 1995)

For regional transportation planning, the background knowledge, data, objectives and evaluation criteria are becoming more and more complex. This trend may also apply to other areas as Schoemaker and Russo (1993) argued that the complexity and the amount of data involved in a decision problem keep increasing over years and the recent technological advances 
is making the decision making even more challenging than ever. MAVT based method does not need to involve any consistency check and the required input data is also less then outranking and pair-wise comparison based methods.

In short, guided by the work of Vincke (1992), the selection of MAVT as the default MCDA method is based on the consideration on the nature of the application area, decision alternatives, criteria, measurement scales, dependency among the criteria, type of uncertainties, and quantity and quality of the available data and judgments.

\subsubsection{The Elicitation of Input Variables}

\section{Elicitation techniques}

How to assess or elicit the required information and values for MCDA models is an important and interesting topic. Bell et al. state that "the art and science of elicitation of values (about consequences) and judgments (about uncertainties) lies at the heart of prescriptive endeavors" (Bell et al, 1988). In this section, we will discuss the selection and best practice of elicitation techniques for criteria importance shifting and construction of future scenarios.

Although there has been an increase in research on elicitation techniques over the last decades within different disciplines such as management science, psychology, and statistics, no methods are generally accepted by researchers and practitioners. To eliciting adequate quantitative information from people is still one of the major challenges in the field of decision analysis (Fox, 2003). The only consensus of these researches is the fact that we should be concerned not only with what experts are asked to evaluate, but also how they are asked. Because the psychological findings on how human-beings think and process uncertain 
information cognitively, and how they respond to queries confirmed the potential bias when elicitation is not well-planned and well-performed.

Selecting techniques for elicitation is a matter of balancing the quality of retrieved information with the resource and cognitive effort will be used for eliciting the required information. The techniques and methods used for elicitation must be practical and should not require too many inputs from the decision-maker(s).

Input variables for scenario planning include: 1) the driving forces used to "assemble" scenario and 2) the stakeholders' value judgments of the system under different scenarios. Considering the participation of multiple stakeholders, objective of the elicitation of these input variables is to identify and clarify the public values. Kenney, Winterfeldt and Eppel (1990) summarized the five approaches can help to illuminate and collect public values in complex policy problems: survey, indirect value elicitation, direct value elicitation, focus groups, and public involvement. The selections of these approaches are case-to-case problems. The remaining of this section will describe the elicitation used in the field of regional transportation planning.

Regional transportation planning is usually conducted periodically by metropolitan planning organizations or the state department of transportation. While conducting scenario planning for these agencies, indirect value elicitation based on existing technical reports, and direct value elicitation techniques are preferred.

Indirect value elicitation based on existing technical reports is usually utilized to identify the driving forces for scenario construction. The advantage of this approach is it re-uses the investigative and decision analysis resources, which were used in distributed technical reports 
regarding different planning aspects. By searching for and summarizing the assumptions, uncertainties and concerns in these materials, we could identify those macro-factors such as economics, politics, technology and etc., which are potentially disruptive to the planning effort. Similarly, indirect value elicitation is also capable of extracting preference model about system states under different scenarios with modest additional effort.

Although indirect value elicitation can be efficient in some cases, it is not realistic to expect all driving forces and stakeholders preference models that are potentially critical to the planning can be obtained without any interaction with decision-makers and stakeholders. Direct value elicitation is an effective approach to elicit input variables to scenario planning.

Direct value elicitation is not particularly designed for the purpose of identifying driving forces. However, the elicitation of driving forces can be conducted via formal methods, such as the Delphi method (Dalkey, 1970) and nominal group techniques (Gustafson et al., 1973). These approaches have been utilized to obtain consensus summaries in a multi-stakeholder environment. Without using such formal methods, scientific panel, such as those of the Intergovernmental Panel on Climate Change is also an alternative approach.

Direct value elicitation for stakeholder preference model consists of interactive conversations with individuals or groups regarding their evaluation of policy options in terms of single attributes and tradeoffs among multiple competing objectives. There are numerous methods for formal value elicitation, such as direct evaluation (Edwards, 1977) and tradeoff methods in the framework of multiattribute utility theory (Keeney and Raiffa, 1976). However, direct value elicitation cannot be considered as a substitute for definitive scientific research. Direct elicitation is subject to a variety of cognitive pitfalls, such as overconfidence and internal inconsistency 
(Schoemaker, 1995). These methods can, instead, contribute to improve the planning process by clarifying the preference model of stakeholders.

\section{Addressing stakeholder conflicts}

Transportation planning and regional planning is group decision making environment. Stakeholders meeting and public involvement are crucial parts for the final plan to be comprehensive and legitimated. In group decision making, stakeholder conflict is a common but difficulty problem. In this section, we will discuss the practice to handle opinion conflicts.

The term "conflict" is usually very vaguely defined in practice. Academia developed some typologies help define the implications of conflict. Burton (1990) argues that there are three kinds of conflict:

1. Management problems. In this situation, stakeholders share interests and values. However, the decision-making process is not properly organized, so that these stakeholders have to solve a problem jointly.

2. Dispute. Stakeholders disagree about the planning and decision-making because of how they feel the decision will satisfy their interests.

3. Value conflict. Stakeholders have disagreement about basic needs or fundamental values.

In the process of scenario planning for public policies, majority of the conflict opinions are type 1 and 2 . Even in a few cases, where stakeholders have fundamental value conflict, conflict opinions are not necessarily unproductive. A group without conflict opinions is usually non responsive to the needs for changes (De Dreu and Van De Villert, 1997). Therefore, theory 
of conflict management distinguishes the concepts of "managing" and "resolve" conflicts (Thompson, 2004). In other words, it is important to ensure a minimum level of conflict to keep in a discussion or group decision making process. Conflict beyond this level calls for approaches to resolve.

There are three categories of methods on the resolution of conflicts: principled negotiation (Fisher and Ury 1991), consensus building (Susskind et al., 1999), and mediation (Moore 2003). All these methods emphasize effective communications and process of pragmatic problem-solving. Two principles are considered essential, regardless of specific method (Fuller and Fritzen, 2007):

\section{Perception of fairness.}

2. Better understanding of each other.

In our case study of regional transportation planning, a filtering process is designed for resolving the conflict opinions on the construction of scenarios. The process does not decline any introduced scenarios at the beginning. After collecting all conflict opinions on scenarios, we first combine those scenarios having same implications in terms of policy analysis. Next, via a public involved evaluation procedure, the significances of these scenarios are quantified via scientific assessments. The scenarios associated with greater disruptiveness are kept for further studies.

On the other hand, the resolution of conflict opinions on the state of the infrastructure appeals to communications under informative context. Mediation and consensus summaries can be used as tool to accelerate the convergence of stakeholder disagreements. If resource limitation or time pressure applies, we could use voting and other approaches to aggregate the opinions. 


\subsubsection{The Use of Expert Opinions}

In this section, we will discuss the advantage and disadvantage of heavily using expert opinions as source of inputs for decision analysis models.

When we elicit expert opinions, we are extracting expertise. Though generally agreed definition of expertise exists in literature, most researchers agree that expertise is multidimensional (Diasio et al., 2009). And the essential part of expertise is the expert knowledge, which have three main components: 1) formal knowledge, 2) practical knowledge, and 3) self-regulative knowledge (Diasio et al., 2009). Formal knowledge is obtained from education, training and other formal learning processes; practical knowledge is more tacit, in which the intuition plays an important role. Thus practical expert knowledge is more difficult to be accurately elicited; self-regulative knowledge is about the reflective skills that individuals use to evaluate their own actions.

Due to methodological and cognitive limitations, expert opinions are sometimes criticized by literatures in descriptive decision field (Kahneman et al., 1982). But the use of decision model and elicitation techniques is always case by case. (Ayyub, 2008)

However in the real-world cases, as discussed in section 3.7, we have to balance the quality of information with the resource used while preparing the input variables for decision models. Ideally, all decisions in regional planning should be supported by evidences from scientific research and modeling. However, research and modeling on complex system, e.g. environmental system and social-economic system can be very resource demanding, especially for the first time analysis. For regional planning organization, research and modeling based decision-making will become almost impossible, for two reasons: 1) when the scale of the 
system is smaller, it is more likely statistical rules and conclusions result in biased conclusions; 2)

the research, modeling and computation resources are usually limited for regional planning agencies. In this context, experts and senior staffs become a reliable and resource-friendly source of the inputs for decision-making.

On the other hand, while elicitation of expert opinions is used to "aid" the decisionmaking process, expert opinions get calibrated through the collaboration and communication. This advantage of involving expert opinions is especially important when the elicitation process has been performed iteratively. Human intelligence makes the feedback and opinion updating very smoothly and efficiently, while typical scientific models may not be able to be corrected with moderate effort if some of the fundamental assumptions are found violated due to the changes in environments.

Additionally, the framework does not prevent the use of research and modeling results. If resources or existing data are available, planners can always incorporate the data as inputs to improve the accuracy and reliability of the conclusions.

\subsection{Mathematical Addendum}

This addendum is the derivation of Eq. (7). We will prove the statement that $\phi$ reaches its maximal value when $\tau_{i}^{k}=n+1-\tau_{i}^{0}, i=1 \ldots n$.

Definition 1 (Ranking sequence) The sequence $\left(\tau_{1}, \tau_{2}, \tau_{3}, \ldots, \tau_{i}, \tau_{i+1}, \ldots, \tau_{n}\right)$, denoted as $\left(\tau_{i}\right), i=1 \ldots n$ is called the ranking sequence of a group of decision alternatives $\left(x_{i}\right), i=1 \ldots n$. The 
ranking sequence obtained in baseline scenario, denoted as $\left(\tau_{i}^{0}\right), i=1 \ldots n$, is called the baseline ranking sequence.

Definition 2 (Rank swap) Considering a pair of decision alternatives $x_{i}$ and $x_{j}$ under a scenario $s_{k}$, the operation assigning $\tau_{i}^{k}=\tau_{j}^{0}, \tau_{j}^{k}=\tau_{i}^{0}$ is called a rank swap between these 2 decision alternatives.

Obviously, any ranking sequence can be obtained by finite rank swaps from a given ranking sequence. The proof is omitted.

Lemma 1 (The condition under which a rank swap increase the value of $\phi$ ) Suppose a given ranking sequence $\left(\tau_{i}^{p}\right), i=1 \ldots n$, is obtained by $p$ rank swap operations from the baseline ranking sequence. After an additional rank swap operation, $\phi^{p+1}>\phi^{p}$ if and only if $\left(\tau_{i}^{p}-\tau_{j}^{p}\right)\left(\tau_{i}^{0}-\tau_{j}^{0}\right)>0$

Proof:

$$
\begin{aligned}
& \phi^{p+1}>\phi^{p} \\
& \Leftrightarrow \lambda \sum_{i=1}^{n}\left(\tau_{i}^{p+1}-\tau_{i}^{0}\right)^{2}-\lambda \sum_{i=1}^{n}\left(\tau_{i}^{p}-\tau_{i}^{0}\right)^{2}>0 \\
& \Leftrightarrow \sum_{i=1}^{n}\left[\left(\tau_{i}^{p+1}-\tau_{i}^{0}\right)^{2}-\left(\tau_{i}^{p}-\tau_{i}^{0}\right)^{2}\right]>0 \\
& \Leftrightarrow \sum_{i=1}^{n}\left[\left(\tau_{i}^{p+1}\right)^{2}+\left(\tau_{i}^{0}\right)^{2}-2 \tau_{i}^{0} \tau_{i}^{p+1}-\left(\tau_{i}^{p}\right)^{2}-\left(\tau_{i}^{0}\right)^{2}+2 \tau_{i}^{0} \tau_{i}^{p}\right]>0 \\
& \Leftrightarrow \sum_{i=1}^{n}\left[\left(\tau_{i}^{p+1}-\tau_{i}^{p}\right)\left(\tau_{i}^{p+1}+\tau_{i}^{p}-2 \tau_{i}^{0}\right)\right]>0
\end{aligned}
$$

Without loss of generality, assume the rank swap operation between $\left(\tau_{i}^{p}\right)$ and $\left(\tau_{i}^{p+1}\right)$ is $\tau_{x}^{p+1}=\tau_{y}^{p}, \tau_{y}^{p+1}=\tau_{x}^{p}$, then 


$$
\begin{aligned}
& \sum_{i=1}^{n}\left[\left(\tau_{i}^{p+1}-\tau_{i}^{p}\right)\left(\tau_{i}^{p+1}+\tau_{i}^{p}-2 \tau_{i}^{0}\right)\right] \\
& =\sum_{i \neq x, y}\left[\left(\tau_{i}^{p+1}-\tau_{i}^{p}\right)\left(\tau_{i}^{p+1}+\tau_{i}^{p}-2 \tau_{i}^{0}\right)\right]+\sum_{i=x, y}\left[\left(\tau_{i}^{p+1}-\tau_{i}^{p}\right)\left(\tau_{i}^{p+1}+\tau_{i}^{p}-2 \tau_{i}^{0}\right)\right] \\
& =0+\left(\tau_{x}^{p+1}-\tau_{x}^{p}\right)\left(\tau_{x}^{p+1}+\tau_{x}^{p}-2 \tau_{x}^{0}\right)+\left(\tau_{y}^{p+1}-\tau_{y}^{p}\right)\left(\tau_{y}^{p+1}+\tau_{y}^{p}-2 \tau_{y}^{0}\right) \\
& =\left(\tau_{y}^{p}-\tau_{x}^{p}\right)\left(\tau_{y}^{p}+\tau_{x}^{p}-2 \tau_{x}^{0}\right)+\left(\tau_{x}^{p}-\tau_{y}^{p}\right)\left(\tau_{x}^{p}+\tau_{y}^{p}-2 \tau_{y}^{0}\right) \\
& =2\left(\tau_{x}^{p}-\tau_{y}^{p}\right)\left(\tau_{x}^{0}-\tau_{y}^{0}\right)
\end{aligned}
$$

So $\phi^{\prime \prime}>\phi^{\prime} \Leftrightarrow\left(\tau_{x}^{p}-\tau_{y}^{p}\right)\left(\tau_{x}^{0}-\tau_{y}^{0}\right)>0$.

Theorem $\phi$ reaches its maximal value when $\tau_{i}^{k}=n+1-\tau_{i}^{0}, i=1 \ldots n$.

Proof: $\forall i, j \in\{1,2, \ldots, n\}$,

$$
\left(\tau_{i}^{k}-\tau_{j}^{k}\right)\left(\tau_{i}^{0}-\tau_{j}^{0}\right)=\left[\left(n+1-\tau_{i}^{0}\right)-\left(n+1-\tau_{j}^{0}\right)\right]\left(\tau_{i}^{0}-\tau_{j}^{0}\right)=-\left(\tau_{i}^{0}-\tau_{j}^{0}\right)^{2} \leq 0
$$

According to Lemma 1, $\phi$ reaches its maximal value when $\tau_{i}^{k}=n+1-\tau_{i}^{0}, i=1 \ldots n$. 


\subsection{Chapter Summary}

This chapter described the analytical framework introduced by this dissertation. Section 3.2 will first described the multicriteria decision analysis model to set the baseline (no scenario) priority for a group of decision alternatives for a planning effort; Section 3.3 and 3.4 explained how to elicit non-structured knowledge to assess the impacts of external stressors incrementally based on the baseline results; Section 3.5 discussed the metrics which can be used to aggregate the priority changes of all decision alternatives when considering a future scenario. These metrics will be used to quantify the impacts of these future scenarios to the system; Section 3.6 extended the scenario influence quantifying method to support analysis from multiple perspectives; At the end of this chapter, Section 3.7 discussed the MCDA model selection and the elicitation of input variable for the proposed analytical framework. 


\section{Chapter 4. Implementation and Case Studies}

\subsection{Chapter Overview}

This chapter will demonstrate the analytical framework introduced in Chapter 3. Section 4.2 will briefly introduce the background of the four cases studies covered in this chapter. Section 4.3 will describe an application on infrastructure vulnerability assessment of a coastal region. Section 4.4 will describe an integration of the methodology with the existing project selection process of long range transportation planning of the Hampton Roads Planning Organization (HRTPO). Section 4.5 will describe how this methodology was applied to address deep uncertainties for a state-wide multimodal policy analysis project. Section 4.6 will demonstrate the integration of geographic information system (GIS) and the approach on identifying vulnerable traffic analysis zones (TAZ). Section 4.7 will describe the integrated 
vulnerability assessment based on the inputs of the four case studies above, which is able to provide insights on system vulnerability from multiple perspectives.

\subsection{Background of the Case Studies}

The four case studies are all performed for the Hampton Roads regions, which includes Norfolk, Virginia Beach, and several other cities is one of the most low-lying and vulnerable metropolitan areas in the United States (Figure 4.2-1). It has a total population of 1.7 million and is prominent for its year-round ice-free harbor. It is home to the largest naval base on the US east coast, and it has numerous other military facilities from agencies such as US Navy, Coast Guard, Air Force, NASA, Marines, and Army. The mission performance of these civil and military facilities and infrastructure could be vulnerable, over a horizon of decades, to manifestations of climate change such as sea level and storm frequency. Among the other transportation assets of this region are two international airports, a number of major bridges and tunnels, and an extensive highway and secondary road network, much of which is maintained by the Virginia Department of Transportation (VDOT). The Virginia Governor's Commission on Climate Change (GCCC, 2008) forecasts an average temperature increase of $3.1^{\circ} \mathrm{C}\left(5.6^{\circ} \mathrm{F}\right)$ for Virginia. Based on the current trend of sea level rise in this region, the U.S. EPA's Chesapeake Bay Program (Pyke et al., 2008) estimates that the Chesapeake Bay will experience sea-level rise (approximately $0.7 \sim 1.6 \mathrm{~m}(2.3 \sim 5.2 \mathrm{ft})$ by 2100$)$, water temperature rise (about $0.3 \mathrm{oC}\left(0.5^{\circ} \mathrm{F}\right)$ per decade) and increased precipitation intensity. According to (GCCC, 2008), the Virginia BeachNorfolk metropolitan statistical area ranks tenth in the world in terms of values of assets exposed to flooding caused by sea-level rise. 
Among the many areas around the world that are facing the challenges from climate change impacts, Hampton Roads is at the top tier in that list. People at that region are already facing these impacts at some level and the magnitude and span of these impacts are projected to rise to an alarming level in the future. Like most of the low-lying coastal regions in US, the region is vulnerable to climate change impacts. Numerous studies have focused on the Hampton Roads to assess the region's vulnerability to climate change (Kleinosky, 2006) (Savonis, 2008) (McFarlane, B.J., 2010). Table 4.2-1 shows the summarized projections of different climate change events specific to the study site.

Please note that as requested by the planning organizations, we will illustrate our analytical framework using fictitious numbers. The importance of this research is not as much in the specific rankings of decision alternatives / scenarios that it suggests as in the methodology for the analysis that it provides. All tables and figures are based on fictitious numbers and do not attempt in any way to represent actual assessment results. 


\section{Table 4.2-1 Summary of projected climate change in the Hampton Roads region:}

\begin{tabular}{|c|c|}
\hline Climate change & Projection \\
\hline Temperature & $\begin{array}{l}\text { According to the } 2008 \text { final report on Climate Change from the } \\
\text { Governor's Commission on Climate Change, based on the IPCC data, } \\
\text { researchers have projected that from } 2000 \text { to } 2009 \text { the average } \\
\text { temperature in Virginia and surrounding area will increase about } 3.1^{\circ} \mathrm{C} \text {. } \\
\text { In the Chesapeake Bay region over the last } 40 \text { years the average and the } \\
\text { maximum annual temperature have increased by more than } 1^{\circ} \mathrm{C} \text {. }\end{array}$ \\
\hline Sea Level Rise & $\begin{array}{l}\text { According to current HRPDC Climate Change draft report, the observed } \\
\text { sea level trends from different NOAA tide gauges ranges from } 3.48 \pm 0.42 \\
\mathrm{~mm} / \text { year to } 6.06 \pm 1.14 \mathrm{~mm} / \text { year which means that sea level will rise } \\
\text { about } 1.14 \text { feet to } 1.98 \text { feet over } 100 \text { years respectively (NOAA } 2011 \text { ). } \\
\text { Chesapeake Bay Program's Scientific and Technical Advisory Committee } \\
\text { projects that sea levels in the Chesapeake Bay region will be } 0.7-1.6 \\
\text { meters higher by } 2100 \text {. }\end{array}$ \\
\hline Storm Surge & $\begin{array}{l}\text { Though Storm surge is not a direct climate change event but due to } \\
\text { increased frequency and severity in hurricanes the Hampton Roads area is } \\
\text { considered highly vulnerable to storm surge caused by hurricanes. } \\
\text { From the SLOSH storm surge output for the eight critical assets the storm } \\
\text { surge height varied from Oft to } 17.1 \mathrm{ft} \text { depending on different } \\
\text { characteristics of the storm (SLOSH display output). }\end{array}$ \\
\hline
\end{tabular}




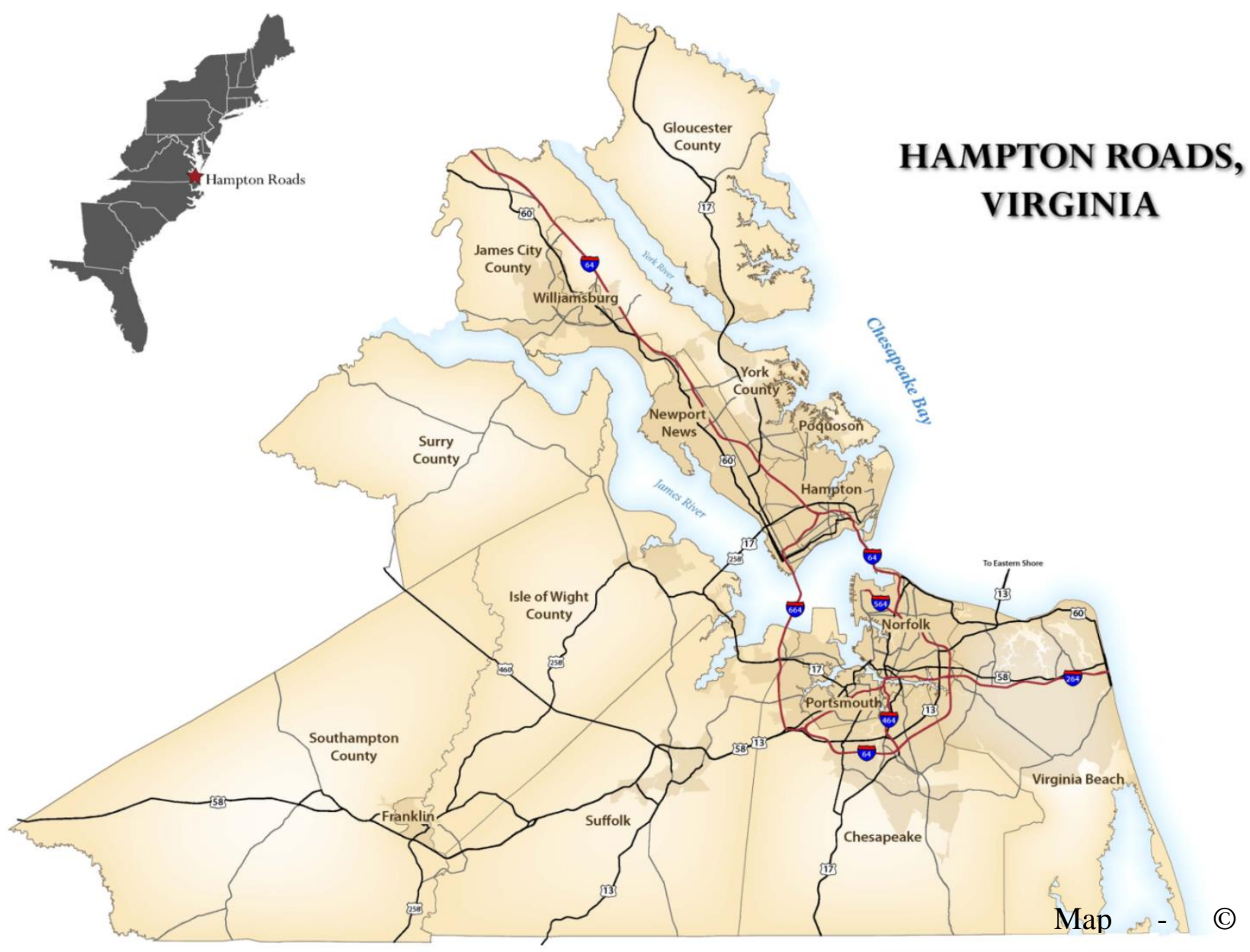

Figure 4.2-1 A map of the region of Hampton Roads, Virginia 


\subsection{Case Study: Infrastructure Assets}

The project is originated from FHWA's office of natural environment. It is a joint effort between the Virginia center for transportation innovation and research (VCTIR), the Hampton Roads Planning District Commission (HRPDC), the University of Virginia Center for Risk Management of engineering system, the University of Virginia Center for Transportation Studies, and the Hampton Roads Transportation Planning Organization (HRTPO).

The goals of the project are:

1. To identify, analyze, and prioritize a comprehensive set of transportation assets in Hampton Roads that have the highest exposure to climate change threats, and have the highest potential for significant impacts due to climate change.

2. To incorporate the consideration of vulnerabilities due to climate change into ongoing work address infrastructure protection.

3. To document lessons learned in applying FHWA's risk assessment model, and to develop specific recommendations for the FHWA to consider in revising the model.

\subsubsection{Overview}

The analysis framework of this paper has been implemented in a software workbook to facilitate real-time interaction and engagement with stakeholders. Applying the framework in a practical situation entails two phases: (i) baseline (no-scenario) analysis and (ii) scenario analysis. Each phase has several worksheets to provide for the transparency of inputs and intermediate and final results. In a single iteration, the workbook can address up to fifty criteria, forty assets, and 
five scenarios. Recognizing that analyzing more criteria, assets or scenarios would increase the cognitive challenge and diminish the transparency of the procedures, thirteen criteria, twelve assets, and three scenarios are used in the demonstration to follow. The various natural-language statements related to the assessment of assets and to criteria importance are obtained and interpreted by conventional elicitation methods (Ayyub, 2001).

The inputs for the variables in the analytical frameworks are obtained from two one-hour stakeholder meetings. The attendees of the meetings include transportation regional planners from HRPDC and HRTPO, researchers on stormwater and flooding management from VCTIR, and faculty members and research assistants of University of Virginia.

\subsubsection{Assets Identification}

VDOT and HRTPO have traditionally maintained a number of inventories, including the VDOT Asset Management System, for maintenance or project programming and planning needs; the GIS integrator, a statewide inventory of VDOT infrastructure and data for general use within VDOT. However, the number of assets owned by a transportation agency is large and diverse in nature. Here, the asset data were retrieved from the VDOT asset management system (AMS) (VDOT, 2006) and PONTIS database (Thompson et al., 2008) and a preliminary list includes thousands of assets. Four screening criteria are used to systematically reduce the scale of the problem to fewer than twenty major assets. These include (1) those assets that are on Hurricane evacuation routes (VDOT, 2011a); (2) those assets that carry high traffic volume (AADT > 10,000 vehicles/day); (3) those assets that represent a maintenance priority route (e.g. snow removal priority route); and (4) those that are at low elevation. These criteria are intended to 
focus the demonstration on a high-level policy analysis on a manageable set of major assets. Three bridges - Chesapeake Bay Bridge-Tunnel, Berkley Bridge and Gilmerton Bridge-are included because of their importance to transporting freight and people (VDOT, 2011b). In addition to the bridges and tunnels, there is particular concern for two traffic management center facilities. Figure 4.3-1 describes the assets selection criteria. Figure 4.3-2 and Table 4.3-1 describe the assets that are selected for the demonstration.

\subsubsection{Criteria Definition}

Cost related factors are usually not included in the criteria, because the analysis is supporting what the agency describes as an unconstrained long-range plan. An unconstrained plan is useful to focus high-level discussion on the needs of the region, without considering the uncertain costs of potential actions. Unconstrained planning is a common activity in large-scale systems. Cost-effectiveness, cost-benefit, and cost-efficiency analyses are typically performed in subsequent or parallel analysis.

The criteria set $\mathrm{C}$ for prioritizing the assets were adapted from the working papers of the regional transportation planning organization (Hampton Roads Planning District Commission, in coordination with the regional Metropolitan Planning Organization) (Case et al., 2007). The criteria are harmonious with the planning factors of the FHWA and with goals of the Safe, Accountable, Flexible and Efficient Transportation Equity Act (SAFETEA).

Table 4.3-2 describes how the criteria appear in the workbook. For demonstration, the weights of criteria under the baseline scenario are set to be equal (Guiasu, S. and Shenitzer, A, 1985). 


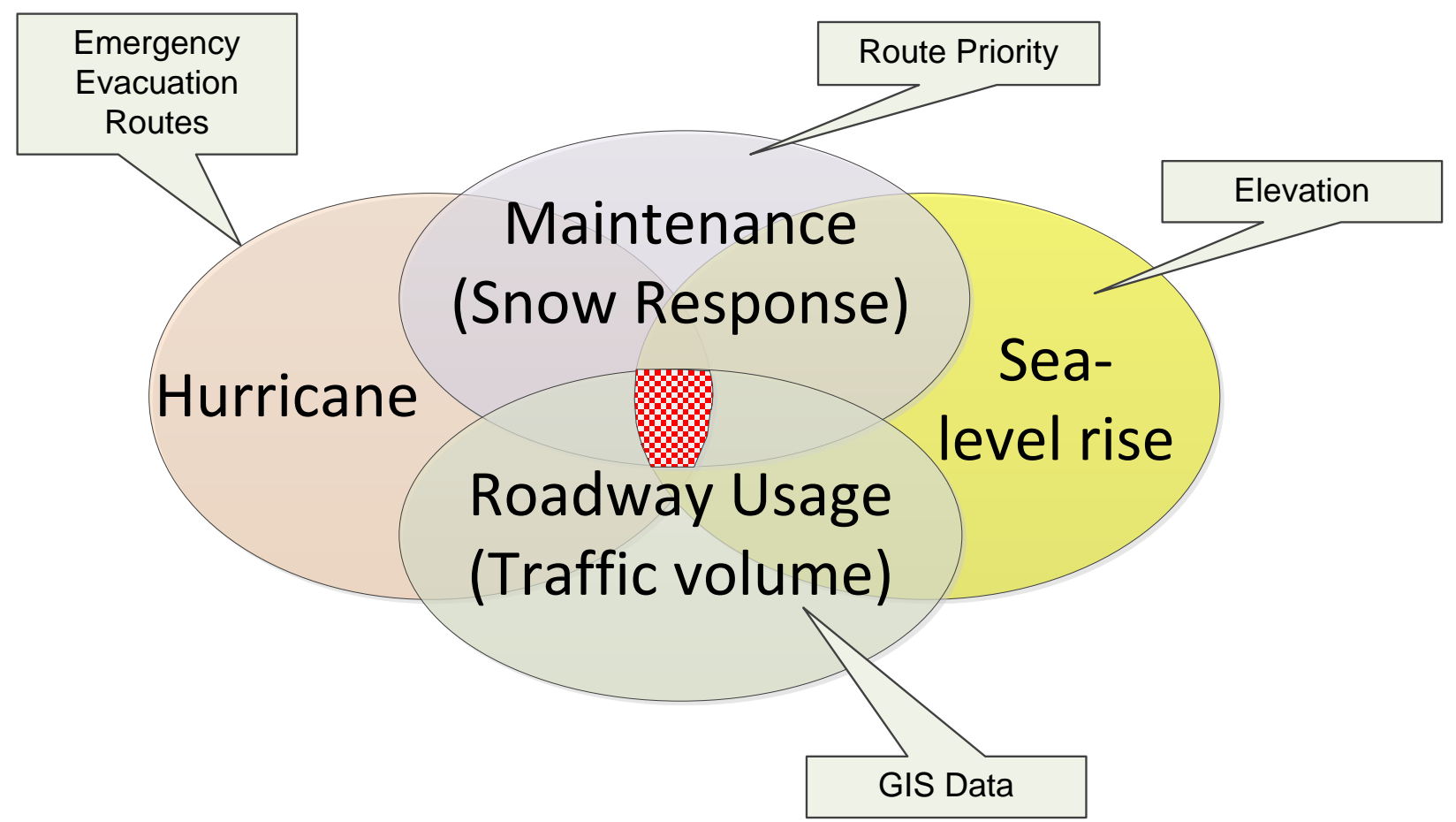

Figure 4.3-1 The selection criteria for the list of infrastructure assets in the case study 4.3 


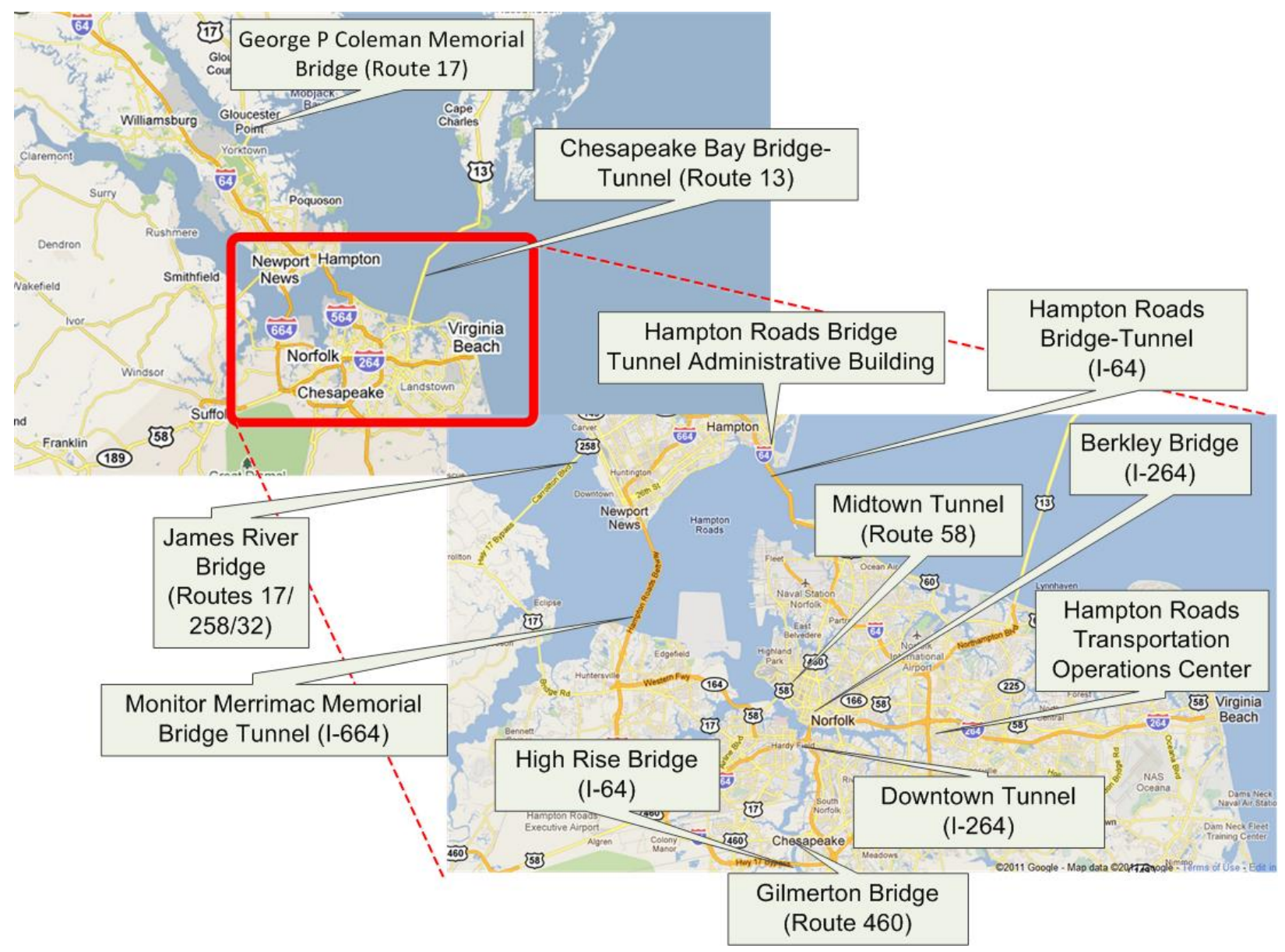

Figure 4.3-2 The selected twelve infrastructure assets for the case study 4.3 
Table 4.3-1 The set of selected transportation assets for the case study 4.3

\begin{tabular}{ll}
\hline \multicolumn{1}{c}{$\boldsymbol{I D}$} & \multicolumn{1}{c}{ Assets } \\
\hline$a_{01}$ & George P Coleman Memorial Bridge \\
$a_{02}$ & James River Bridge \\
$a_{03}$ & Hampton Roads Bridge-Tunnel \\
$a_{04}$ & Monitor Merrimac Memorial Bridge-Tunnel \\
$a_{05}$ & Midtown Tunnel \\
$a_{06}$ & High Rise Bridge \\
$a_{07}$ & Downtown Tunnel \\
$a_{08}$ & Hampton Roads Bridge Tunnel Administrative Building \\
$a_{09}$ & Hampton Roads Transportation Operations Center \\
$a_{10}$ & Chesapeake Bay Bridge-Tunnel \\
$a_{11}$ & Berkley Bridge \\
$a_{12}$ & Gilmerton Bridge \\
\hline
\end{tabular}


Table 4.3-2 A comprehensive set of performance criteria for the case study $4.3^{6}$.

\begin{tabular}{|c|c|}
\hline Criterion & Descriptions \\
\hline $\mathrm{c}_{01}$. Public involvement & $\begin{array}{l}\text { Enhance public involvement in the development of the } \\
\text { region's transportation system. }\end{array}$ \\
\hline $\mathrm{c}_{02}$. Regional perspective & $\begin{array}{l}\text { Include a regional perspective among the transportation } \\
\text { prioritization criteria. }\end{array}$ \\
\hline $\mathrm{c}_{03}$. Fiscal responsibility & $\begin{array}{l}\text { Develop a long-range transportation plan that is fiscally } \\
\text { constrained. }\end{array}$ \\
\hline $\mathrm{c}_{04 .}$ Economic vitality & $\begin{array}{l}\text { Support the economic vitality of the region, emphasizing } \\
\text { global competitiveness, productivity, and efficiency. }\end{array}$ \\
\hline$c_{05}$. Safety & $\begin{array}{l}\text { Increase the safety of the transportation system for } \\
\text { motorized and non-motorized users. }\end{array}$ \\
\hline $\mathrm{c}_{06}$. Security & $\begin{array}{l}\text { Provide for the security of the regional system for motorized } \\
\text { structure and its users. }\end{array}$ \\
\hline $\mathrm{c}_{07}$. Accessibility and mobility & Increase accessibility and mobility of people and goods. \\
\hline $\mathrm{c}_{08}$. Environment & $\begin{array}{l}\text { Protect and enhance the environment, promote energy } \\
\text { conservation, improve quality of life, and reduce } \\
\text { greenhouse gas emissions. }\end{array}$ \\
\hline $\begin{array}{l}\mathrm{c}_{09} . \text { Compatibility with land } \\
\text { use and economic patterns }\end{array}$ & $\begin{array}{l}\text { Obtain compatibility between transportation improvements } \\
\text { and planned land use and economic development patterns. }\end{array}$ \\
\hline $\begin{array}{l}\mathrm{c}_{10} . \text { Modal integration and } \\
\text { connectivity }\end{array}$ & $\begin{array}{l}\text { Enhance the integration and connectivity of the } \\
\text { transportation system, across and between modes, for } \\
\text { people and goods. }\end{array}$ \\
\hline $\begin{array}{l}\mathrm{c}_{11} . \text { Management and } \\
\text { operation }\end{array}$ & $\begin{array}{l}\text { Optimize the efficient system management and operation of } \\
\text { the regional transportation system. }\end{array}$ \\
\hline $\begin{array}{l}c_{12} . \text { Maintenance and } \\
\text { replacement }\end{array}$ & $\begin{array}{l}\text { Increase the optimization, maintenance and replacement of } \\
\text { the existing transportation system. }\end{array}$ \\
\hline$c_{13}$. Revenue source & $\begin{array}{l}\text { Work toward finding dedicated and sustainable revenue } \\
\text { sources for transportation. }\end{array}$ \\
\hline
\end{tabular}

${ }^{6}$ Adapted from 2034 Hampton Roads Long-Range Transportation Plan (HRTPO, 2011) for the consideration of infrastructure policies and investments 


\subsubsection{Baseline Scenario Multicriteria Analysis}

With the criteria and assets already defined, the framework requires input of experts to complete the baseline scenario assessment. The baseline scenario aims to prioritize assets assuming no-scenario climate conditions (no climate change is yet considered). Table 4.3-3 describes the recording of expert inputs on the ratings across the set of criteria under the baseline scenario. The expert inputs are in terms of natural-language statements (strongly agree, agree, somewhat agree, indifferent, and disagree or not relevant). Across the criteria, c01, c09 and c13 are considered not relevant to the prioritization. The expert inputs were quantified and aggregated via a weighted sum to obtain scores and corresponding prioritization of assets under the baseline scenario. Table 4.3-4 shows the score and priorities resulting rank order of the assets under the baseline scenario.

\subsubsection{Scenario Definitions}

Table 4.3-5 demonstrates that climate change scenarios were developed and incorporated to adjust the baseline prioritization of assets. The reader is referred to (Wu et al., 2011) for more details on scenario development. Experts and stakeholders were invited to adjust the criteria importance per scenario, as follows. Climate change scenarios are constructed by combining a variety of climate change conditions. These conditions describe climate change events that would be expected to influence the prioritization of the assets, such as sea-level rise, increase in storm surge, increase in storm frequency, etc. The demonstration addressed three scenarios. The

first two scenarios consist of adverse-impact conditions, to capture the effects brought by plausible future climate change. The first scenario, s1, is an extreme scenario, consisting of the 
most adverse conditions of climate change and natural disasters. The second scenario, s2, is a milder scenario. This scenario considers climate change conditions-sea-level rise, land subsidence - are becoming worse, while other climate conditions are unchanged. The third scenario, s3, a non-extreme scenario, represents a situation in which climate change would slow down. Table 4.3-5 describes the construction of the three scenarios via the software workbook.

\subsubsection{Criteria Importance Adjustment for New Scenarios}

The adjustment of the relative criteria importance is a key innovation of this study. The weights of criteria were changed (re-weighted) based on each of the several climate scenarios. Table 4.3-6 shows a summary of how the criteria importance varied with the scenarios. The importance changes were accomplished by natural-language statements of major increase, major decrease, minor increase and minor decrease to the criteria. A blank cell means no change of relative importance. Based on these statements, the incremental weights adjustment of a previous section was used to generate new weights for these criteria under each scenario. 
Table 4.3-3 The twelve selected assets (top row) are assessed on thirteen criteria (left column) in the case study 4.3.

\begin{tabular}{|c|c|c|c|c|c|c|c|c|c|c|c|c|}
\hline \multirow{2}{*}{ Criterion } & \multicolumn{12}{|c|}{ Asset } \\
\hline & $a_{01}$ & $a_{02}$ & $a_{03}$ & $a_{04}$ & $a_{05}$ & $a_{06}$ & $a_{07}$ & $a_{08}$ & $a_{09}$ & $a_{10}$ & $a_{11}$ & $a_{12}$ \\
\hline$c_{01}$ & N/A & N/A & N/A & N/A & N/A & N/A & N/A & N/A & N/A & N/A & N/A & N/A \\
\hline$c_{02}$ & $\mathrm{Agr}$ & Agr & StrAgr & $\mathrm{Agr}$ & SomAgr & Agr & StrAgr & $\mathrm{Agr}$ & Agr & StrAgr & SomAgr & Agr \\
\hline$c_{03}$ & SomAgr & Agr & Agr & Agr & Agr & Agr & Agr & Agr & Agr & Agr & Agr & $\mathrm{Agr}$ \\
\hline$c_{04}$ & SomAgr & Agr & StrAgr & StrAgr & StrAgr & Agr & StrAgr & Agr & Agr & StrAgr & Agr & Agr \\
\hline$c_{05}$ & Agr & SomAgr & Agr & Agr & Agr & Agr & Agr & $\mathrm{Agr}$ & StrAgr & $\mathrm{Agr}$ & SomAgr & Agr \\
\hline$c_{06}$ & SomAgr & Agr & Agr & StrAgr & Agr & Agr & $\mathrm{Agr}$ & SomAgr & SomAgr & StrAgr & Agr & SomAgr \\
\hline$c_{07}$ & Agr & Agr & StrAgr & StrAgr & Agr & Agr & Agr & StrAgr & StrAgr & StrAgr & Agr & Agr \\
\hline$c_{08}$ & Agr & SomAgr & Agr & Agr & Agr & SomAgr & Agr & SomAgr & SomAgr & StrAgr & Agr & Agr \\
\hline$c_{09}$ & N/A & N/A & N/A & N/A & N/A & N/A & N/A & N/A & N/A & N/A & N/A & N/A \\
\hline$c_{10}$ & Agr & Agr & StrAgr & StrAgr & StrAgr & Agr & StrAgr & SomAgr & Agr & Agr & SomAgr & SomAgr \\
\hline$c_{11}$ & Agr & SomAgr & Agr & Agr & SomAgr & Agr & Agr & StrAgr & StrAgr & Agr & Agr & SomAgr \\
\hline$c_{12}$ & SomAgr & SomAgr & Agr & Agr & Agr & SomAgr & SomAgr & StrAgr & StrAgr & Agr & SomAgr & Agr \\
\hline$c_{13}$ & N/A & N/A & N/A & N/A & N/A & N/A & N/A & N/A & N/A & N/A & N/A & N/A \\
\hline
\end{tabular}

* The assessment levels are: strongly agree (StrAgr), agree (Agr), somewhat agree (SomAgr), disagree or not relevant (N/A) 
Table 4.3-4 The baseline scores and rankings of selected assets in the case study 4.3.

\begin{tabular}{ccc}
\hline Assets & \multicolumn{2}{c}{ Baseline Scenario s } \\
Score & Ranking \\
\hline $\mathrm{a}_{01}$ & 41 & 11 th \\
$\mathrm{a}_{02}$ & 41 & 10 th \\
$\mathrm{a}_{03}$ & 62 & $2 \mathrm{nd}$ \\
$\mathrm{a}_{04}$ & 62 & $2 \mathrm{nd}$ \\
$\mathrm{a}_{05}$ & 51 & 7 th \\
$\mathrm{a}_{06}$ & 46 & 8 th \\
$\mathrm{a}_{07}$ & 56 & 4 th \\
$\mathrm{a}_{08}$ & 51 & 6 th \\
$\mathrm{a}_{09}$ & 56 & 4 th \\
$\mathrm{a}_{10}$ & 64 & $1 \mathrm{st}$ \\
$\mathrm{a}_{11}$ & 41 & 11 th \\
$\mathrm{a}_{12}$ & 44 & 9 th \\
\hline
\end{tabular}

Table 4.3-5 Scenario definitions in the case study 4.3

\begin{tabular}{lcccc}
\hline \multicolumn{1}{c}{ Conditions } & \multicolumn{3}{c}{ Scenarios } \\
& $\mathrm{s}_{0}$ & $\mathrm{~s}_{1}$ & $\mathrm{~s}_{2}$ & $\mathrm{~s}_{3}$ \\
\hline Sea level rise $<1 \mathrm{~m}$ & & & & $\mathrm{x}$ \\
Sea level rise $>1 \mathrm{~m}$ & $\mathrm{x}$ & $\mathrm{x}$ & \\
Increase in storm water & $\mathrm{x}$ & & \\
Decrease in storm water & & & $\mathrm{x}$ \\
Increase in wave height & $\mathrm{X}$ & & \\
Storm surges & $\mathrm{x}$ & & \\
Subsidence & $\mathrm{x}$ & $\mathrm{x}$ & \\
Decreased erosion & & & $\mathrm{x}$ \\
Increased flooding & & $\mathrm{x}$ & & \\
Decreased flooding & & & $\mathrm{x}$ \\
\hline
\end{tabular}


Table 4.3-6 Three scenarios (top row) and their shift in importance for the thirteen criteria (left column) in the case study 4.3.

\begin{tabular}{|c|c|c|c|}
\hline \multirow{2}{*}{ Criteria } & \multicolumn{3}{|c|}{ Scenarios } \\
\hline & $\mathrm{s}_{1}$ & $\mathrm{~S}_{2}$ & $\mathrm{~S}_{3}$ \\
\hline \multicolumn{4}{|l|}{$\mathrm{c}_{01}$. Public involvement } \\
\hline $\mathrm{c}_{02}$. Regional perspective & Major decrease & & Minor increase \\
\hline $\mathrm{c}_{03}$. Fiscal responsibility & & & Minor increase \\
\hline $\mathrm{c}_{04}$. Economic vitality & Major increase & Minor increase & \\
\hline $\mathrm{c}_{05}$. Safety & & Minor increase & \\
\hline $\mathrm{c}_{06}$. Security & Minor increase & & \\
\hline $\mathrm{c}_{07}$. Accessibility and mobility & & & Minor decrease \\
\hline $\mathrm{c}_{08}$. Environment & & & Minor increase \\
\hline \multicolumn{4}{|c|}{$\mathrm{c}_{09}$. Compatibility with land use and economic patterns } \\
\hline $\mathrm{c}_{10}$. Modal integration and connectivity & Minor increase & & Major increase \\
\hline $\mathrm{c}_{11}$. Management and operation & & Minor increase & Minor decrease \\
\hline $\mathrm{c}_{12}$. Maintenance and replacement & Major decrease & & Minor increase \\
\hline$c_{13}$. Revenue source & & & \\
\hline
\end{tabular}

* The levels of shift are marge increase, marge decrease, minor increase, minor decrease, and no change (blank) 


\subsubsection{Results}

Table 4.3-7 shows the implications of considering climate scenarios in terms of the scores and the rankings of the assets. The result under baseline scenario s0 is described in the first two columns. The scores and rankings for scenarios from s1 to s3, which are obtained through the reweighted additive value functions, reflect the effects of expert adjustments on the relative criteria importance under the scenarios of climate change. For example, asset a08 - Hampton Roads Bridge Tunnel Administrative Building scores a ' 51 ' in the baseline scenario s0, a ' 65 ' in scenario s1 - extreme climate change scenario, a ' 60 ' in scenario s2 - sea-level rise only scenario, , and a ' 62 ' in scenario $\mathrm{s} 3$ - mild climate change scenario.

Several implications can be identified from the corresponding rankings of assets. For example, asset a08 - Hampton Roads Bridge Tunnel Administrative Building is considered to be significantly more important in the scenarios of s3 -sea-level rise only scenario. For scenario s1 -extreme climate change scenario and s2 -sea-level rise only scenario, the priority of this asset among the set of selected assets was approximately constant. This interpretation provided insight to a shifting of asset-management priorities across scenarios. By investigating the source of these different results in terms of criteria weights and assets ratings, the results also allow decision makers to understand how the priorities for asset management can vary with climate change scenarios that are special to the region.

Figure 4.3-3 illustrates the range of scores for the assets across the scenarios. The diamond represents the score of the asset in the baseline scenario. The maximum and minimum scores across the other scenarios are represented by the vertical bars extending from the diamonds. A taller sensitivity bar suggests a greater influence of scenarios on the asset. The sub- 
bars extending from the baseline scenario also can be considered as representing the effect of the re-weighting of the assets assessment criteria. Several of the criteria re-weightings increase and decrease asset scores equally, for example asset a05 - Midtown Tunnel and asset a12 Gilmerton Bridge. It indicates that the re-weighting give potential opportunity and risk to the baseline score. Most of the re-weightings increase and decrease the baseline scores unequally. For example, the re-weighting only results in the increase of aggregated score of asset a03, a08, and a09. Other than the direction of the impact of re-weighting brought by different scenarios, the magnitudes of the impacts of re-weighting also differs. The score of asset a03 is less affected compared to a08 and a09. The above observations are potentially important to understand the degree of consensus for asset management priorities across the scenarios. Another important observation from Figure 5(a) is that many of the vertical bars are overlapping. This indicates that there exist no assets that are dominant in the rankings across all scenarios.

Figure 4.3-4 shows the fluctuation of the asset rankings (priority order) across the scenarios. The ordinal information is not a lossless summary of corresponding cardinal information, and rankings do not maintain the full information of the asset scores. However, rankings make assets more distinguishable from one another, relative to the cardinal scores. Moreover, from a requirement for prioritization, rankings are the preferred form of the output.

There are two important observations from Figure 5(b). First, a majority of the assets only have either upside or downside deviation under multiple scenarios relative to their baseline ranking. For example, asset a10 - Chesapeake Bay Bridge-Tunnel, a03 - Hampton Roads Bridge-Tunnel, a04 - Monitor Merrimac Memorial Bridge-Tunnel, and a07 - Downtown Tunnel have downside sensitivity only. Assets such as a08 - Hampton Roads Bridge Tunnel Administrative Building and a06 - High Rise Bridge do not have downside sensitivity. Second, 
the assets can be divided into two tiers in terms of the range of their rankings. The assets that rank from 1st to 6th, that is, assets a10, a03, a04, a05, a08, and a09 are the first tier. The remaining assets, a02, a06, a07, a10, a11 and a12, comprise a second tier. The assets in the first tier rank between 1st and 7th under the baseline and the constructed climate change scenarios. The second tier assets all ranks no higher than 6th. Identifying the tiers can potentially help decision makers to make robust allocation of resources to assets. With no additional information, a focus of resources on the first tier of assets might be reasonable. 
Table 4.3-7 The scores and rankings of selected assets across the baseline and additional three climate scenarios in the case study 4.3

\begin{tabular}{ccccccccc}
\hline & \multicolumn{7}{c}{ Scenarios } \\
Assets & \multicolumn{2}{c}{$\mathrm{s}_{0}$} & \multicolumn{2}{c}{$\mathrm{s}_{1}$} & \multicolumn{2}{c}{$\mathrm{s}_{2}$} & \multicolumn{2}{c}{$\mathrm{s}_{3}$} \\
& Scores & Ranking & Scores & Ranking & Scores & Ranking & Scores & Ranking \\
\hline $\mathrm{a}_{01}$ & 41 & 11 th & 46 & 10 th & 44 & 10 th & 44 & 10 th \\
$\mathrm{a}_{02}$ & 41 & 10 th & 43 & 12 th & 41 & 12 th & 40 & 11 th \\
$\mathrm{a}_{03}$ & 62 & 2 nd & 66 & 4 th & 63 & 4 th & 61 & 4 th \\
$\mathrm{a}_{04}$ & 62 & 2 nd & 73 & 3 rd & 67 & 2 nd & 61 & 4 th \\
$\mathrm{a}_{05}$ & 51 & 7 th & 59 & 6 th & 54 & 7 th & 46 & 9 th \\
$\mathrm{a}_{06}$ & 46 & 8 th & 53 & 8 th & 51 & 8 th & 53 & 7 th \\
$\mathrm{a}_{07}$ & 56 & 4 th & 56 & 7 th & 57 & 6 th & 60 & 6 th \\
$\mathrm{a}_{08}$ & 51 & 6 th & 65 & 5 th & 60 & 5 th & 64 & 2 nd \\
$\mathrm{a}_{09}$ & 56 & 4 th & 73 & 2 nd & 67 & 2 nd & 67 & 1 st \\
$\mathrm{a}_{10}$ & 64 & 1 st & 74 & 1 st & 68 & 1 st & 62 & 3 rd \\
$\mathrm{a}_{11}$ & 41 & 11 th & 45 & 11 th & 44 & 10 th & 49 & 8 th \\
$\mathrm{a}_{12}$ & 44 & 9 th & 51 & 9 th & 46 & 9 th & 39 & 12 th \\
\hline
\end{tabular}

$\mathrm{s}_{0}-$ baseline scenario;

$\mathrm{s}_{1}$ - extreme climate change scenario;

$\mathrm{s}_{2}-$ Sea-level Rise dominated scenario;

$\mathrm{s}_{3}-$ mild climate change scenario. 


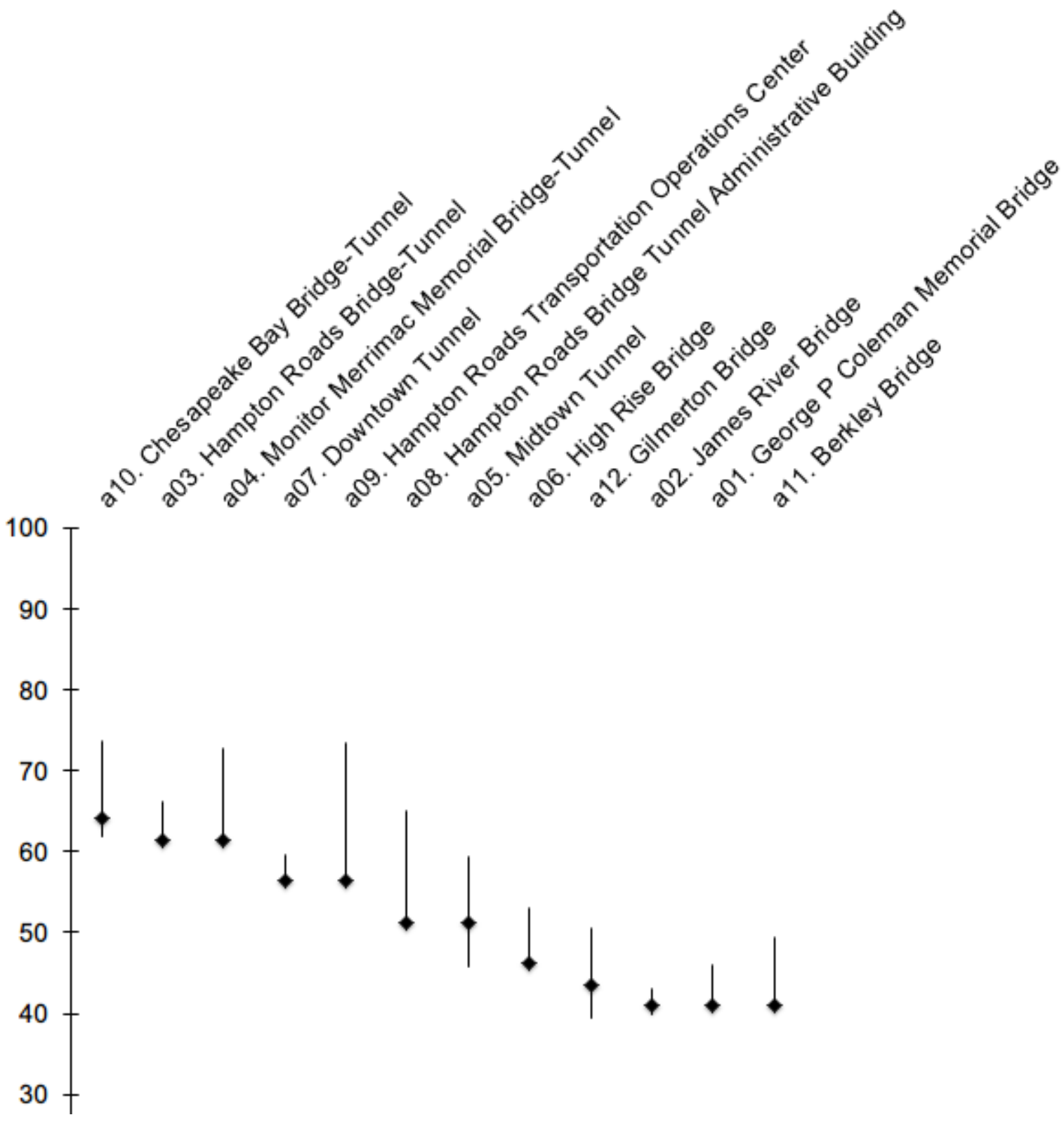

Figure 4.3-3 The baseline scores (diamonds) and the ranges (vertical bars) of scores associated to the three additional climate scenarios of the twelve infrastructure assets addressed in the demonstration. 


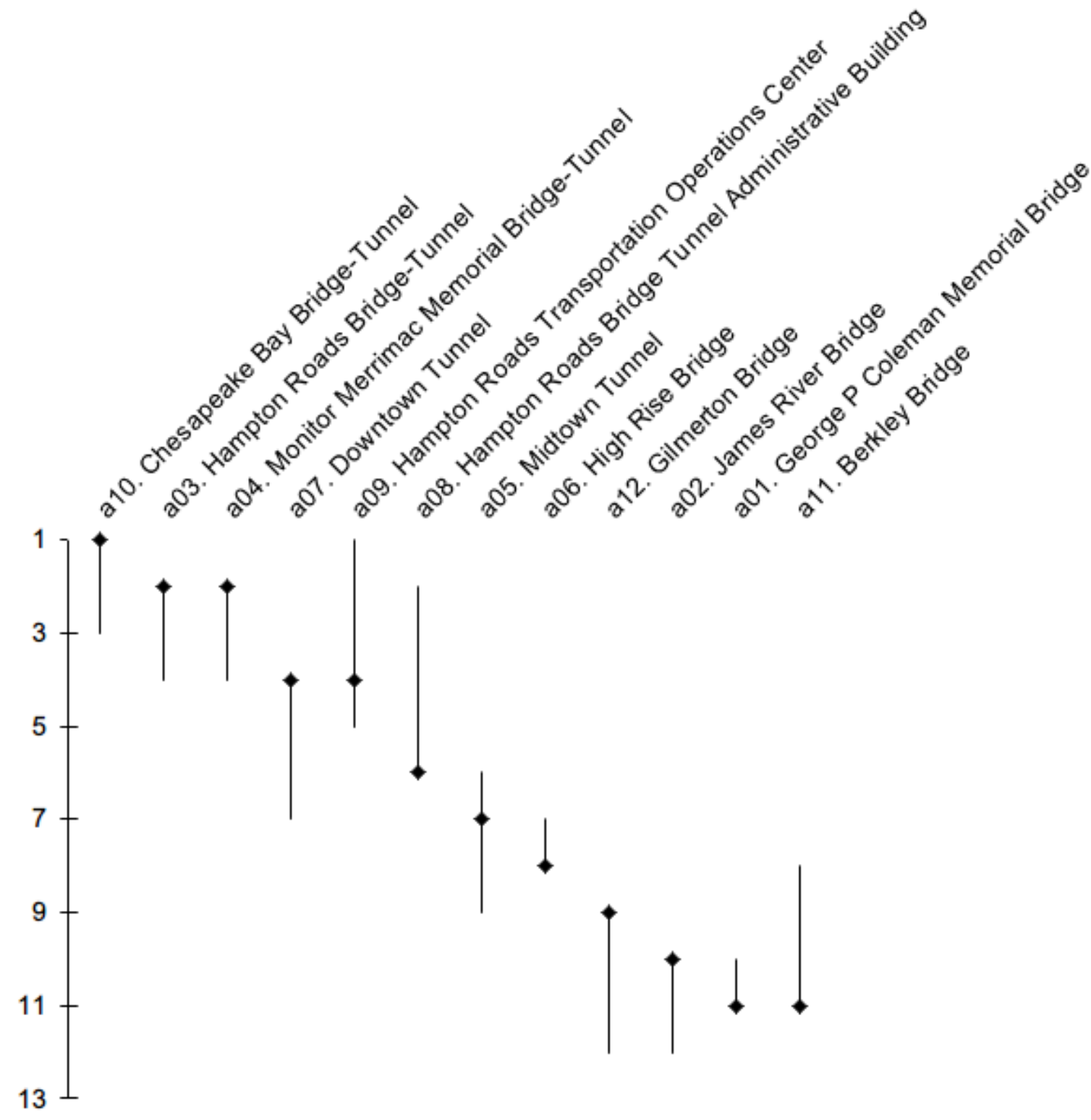

Figure 4.3-4 The baseline rankings (diamonds) and the ranges (vertical bars) of rankings associated to the three additional climate scenarios of the twelve infrastructure assets addressed in the demonstration. 


\subsection{Case Study: Transportation Projects}

\subsubsection{Overview}

This case study is supportive of recent efforts of the US Department of Transportation (USDOT) Federal Highway Administration to address climate change for future transportation investments. The authors are members of a team addressing climate change and transportation systems in the Hampton Roads Region of Virginia, USA. With an estimated total construction cost of approximately $\$ 30$ billion and just over $\$ 7$ billion identified to fund construction, HRTPO staff had the challenging task of evaluating and prioritizing these 150 candidate transportation projects. This case study is based on the project selection and prioritization process.

The transportation planning organization of the Hampton Roads region (HRTPO) conducts priority-setting on transportation investments for its federally mandated thirty-year long-range transportation plan, in part by using a multicriteria analysis. The candidate projects are evaluated across three major criteria and fifteen sub-criteria. The candidate future projects are re-ranked approximately every four years by their aggregated total scores (HRTPO, 2011a).

However, this multicriteria analysis has not yet considered the influence of climate change, among other deep uncertainties. That is, the priority order of candidate projects and portfolio selection is valid for a consensus projection and related, possibly tacit, assumptions regarding the natural environment and the socio-economic system. This makes the strategic portfolio selection decision vulnerable to deep uncertainties, particularly for a long planning horizon of thirty years. Therefore, a methodology helping the agency to address deep uncertainties, including climate change, in the planning effort is desirable. 
This section demonstrates the introduced framework for the Hampton Roads Transportation Planning Organization (TPO/MPO) and its year 2034 long-range plan. The demonstration shows the framework is capable of integrating the long-range plan with the diverse nature of science projections about climate change in the Hampton Roads region (HRPDC, 2010; 2011), and exploring the robustness and sensitivity of the priority order of projects in the long-range plan.

The inputs for the variables in the analytical frameworks are obtained from a one-hour stakeholder meeting and two webinars. The attendees of the meetings include staff from FHWA, transportation regional planners from HRPDC and HRTPO, researchers on stormwater and flooding management from VCTIR, and faculty members and research assistants of University of Virginia.

\subsubsection{Identifying projects, criteria, and baseline value assessments}

Table 4.4-1 provides a sample (about 40 projects) of the 93 candidate regional highway projects in the long range plan of the MPO/TPO. All 93 projects will be analyzed ultimately below, however to provide the overview details would make the table overly long. The projects are updated every four years by member organizations of HRTPO entailing significant public involvement. A multicriteria project prioritization has been used in recent years by the HRTPO for transparency of decision making (HRTPO, 2011a). The criteria include fundamental requirements of transportation planning, including connectivity, safety/security, economic vitality, etc. The criteria are indexed and have measurement scales with maximum possible scores for each of the criteria. With some normalization that converts maximum scores to weights, the 
prioritization is equivalent to Eq. (2), of course preserving the HRTPO assessments and criteria. Table 4.4-2 provides the list of criteria and their respective weights for a baseline (i.e., no scenarios) situation. Prior to our involvement, the HRTPO had assessed each project on each of the criteria via engineering judgment, and developed weights (in terms of maximum point scores) on the criteria through public involvement (HRTPO, 2011b). Table 3 provides the assessments of the 93 projects on 15 criteria. Table 4 displays the aggregated score, according to Eq. (2), and corresponding priority order of a sample (for the sake of brevity) of the 93 candidate projects. 
Table 4.4-1 A sample of the 93 candidate regional highway projects in the case study $4.4^{7}$.

\begin{tabular}{llll}
\hline ID & Projects & ID & Projects \\
\hline$p_{1}$ & Airport Access Road & $p_{11}$ & Woodlake Drive Extended \\
$p_{2}$ & Butts Station Road & $p_{12}$ & Coliseum Drive Connector \\
$p_{3}$ & Cedar Road & $p_{13}$ & Saunders Road \\
$p_{4}$ & Route 17 (G.W. Memorial Highway) & $p_{14}$ & Wythe Creek Road \& Bridge \\
$p_{5}$ & Hanbury Road & $p_{15}$ & Atkinson Boulevard Phase 1 \\
$p_{6}$ & Mt. Pleasant Road, Phase 1 & $p_{16}$ & Harpersville Road \\
$p_{7}$ & Mt. Pleasant Road, Phase 2 & $p_{17}$ & Independence Blvd \\
$p_{8}$ & Portsmouth Boulevard & $p_{18}$ & J. Clyde Morris Blvd (Route 17) \\
$p_{9}$ & Waters Road, Phase 1 & $p_{19}$ & Jefferson Avenue (Route 143) \\
$p_{10}$ & Waters Road, Phase 2 & $p_{20}$ & Liberty Parkway \\
$\ldots \ldots$ & $\ldots . .$. & & \\
$p_{60}$ & Falls Road, Phase 2 & $p_{61}$ & Dam Neck Road \\
$\ldots \ldots$ & $\ldots . .$. & & \\
$p_{74}$ & Princess Anne Road, Phase 1 & $p_{84}$ & Shore Drive \\
$p_{75}$ & Princess Anne Road, Phase 2 & $p_{85}$ & Silina Drive \\
$p_{76}$ & Providence Road & $p_{86}$ & South Independence Blvd \\
$p_{77}$ & Rosemont Road, Phase 1 & $p_{87}$ & Virginia Beach Blvd \\
$p_{78}$ & Rosemont Road, Phase 2 & $p_{88}$ & West Crossing of I-264 \\
$p_{79}$ & Salem Road, Phase 1 & $p_{89}$ & West Neck Pkwy Ext'd, Phase 1 \\
$p_{80}$ & Salem Road, Phase 2 & $p_{90}$ & West Neck Pkwy Ext'd, Phase 2 \\
$p_{81}$ & Sandbridge Road & $p_{91}$ & West Neck Road \\
$p_{82}$ & Seaboard Road, Phase 1 & Witchduck Road \\
$p_{83}$ & Seaboard Road, Phase 2 & Ironbound Road (Rt. 615) \\
\hline & & & \\
\hline & & & \\
& & &
\end{tabular}

${ }^{7}$ Selected from the long-range plan of the metropolitan/transportation planning organization (MPO/TPO) (HRTPO, 2011a). 
Table 4.4-2 A comprehensive set of performance in the case study $4.4^{8}$.

\begin{tabular}{|c|c|c|c|}
\hline Criteria & ID & Max Scores & Weights \\
\hline \multicolumn{4}{|c|}{ Project Utility } \\
\hline Congestion Level & PU-HW.C1 & 30 & 0.100 \\
\hline System Continuity and Connectivity & PU-HW.C2 & 25 & 0.083 \\
\hline Cost Effectiveness & PU-HW.C3 & 15 & 0.050 \\
\hline Compatibility with Existing Plans & PU-HW.C4 & 10 & 0.033 \\
\hline Safety and Security & PU-HW.C5 & 15 & 0.050 \\
\hline Infrastructure Condition & PU-HW.C6 & 0 & 0.000 \\
\hline Modal Enhancements & PU-HW.C7 & 5 & 0.017 \\
\hline \multicolumn{4}{|c|}{ Project Viability } \\
\hline Additional Funding & PV-ALL.C1 & 50 & 0.167 \\
\hline Prior Commitment & PV-ALL.C2 & 10 & 0.033 \\
\hline Federal Mandates & PV-ALL.C3 & 0 & 0.000 \\
\hline Project Readiness & PV-ALL.C4 & 40 & 0.133 \\
\hline \multicolumn{4}{|c|}{ Economic Vitality } \\
\hline Total Reduction in Travel Time & EV-HWBT.C1 & 30 & 0.100 \\
\hline Labor Market Access & EV-HWBT.C2 & 20 & 0.067 \\
\hline Addresses the Needs of Basic Sectors & EV-HWBT.C3 & 30 & 0.100 \\
\hline Increased Opportunity & EV-HWBT.C4 & 20 & 0.067 \\
\hline
\end{tabular}

${ }^{8}$ Adapted from the long-range plan of the metropolitan/transportation planning organization (MPO/TPO) (HRTPO, 2011a). 
Table 4.4-3 A sample of the baseline value assessment of the 93 candidate transportation projects in case study $4.4^{9}$.

\begin{tabular}{|c|c|c|c|c|c|c|c|c|c|c|c|c|c|c|c|c|c|c|}
\hline \multirow{2}{*}{ Criteria } & \multirow{2}{*}{$\begin{array}{l}\text { Max } \\
\text { Scores }\end{array}$} & \multicolumn{17}{|c|}{ Projects } \\
\hline & & $p_{1}$ & $p_{2}$ & $p_{3}$ & $p_{4}$ & $p_{5}$ & $p_{6}$ & $p_{7}$ & $\ldots$ & $p_{61}$ & $\ldots$ & $p_{86}$ & $p_{88}$ & $p_{89}$ & $p_{90}$ & $p_{91}$ & $p_{92}$ & $p_{93}$ \\
\hline PU-HW.C1 & 30 & 21.9 & 13.8 & 21.5 & 20 & 0.8 & 6.3 & 25.9 & & 30 & & 7.5 & 1.2 & 26 & 10.5 & 26 & 16 & 12.5 \\
\hline PU-HW.C2 & 25 & 25 & 25 & 25 & 25 & 8.2 & 8.2 & 16.7 & & 16.7 & & 25 & 8.2 & 25 & 25 & 25 & 16.7 & 25 \\
\hline PU-HW.C3 & 15 & 15 & 14.1 & 6.8 & 6.9 & 0 & 15 & 15 & & 13.1 & & 15 & 15 & 10.7 & 7.7 & 13.1 & 10.6 & 15 \\
\hline PU-HW.C4 & 10 & 10 & 10 & 10 & 10 & 10 & 10 & 10 & & 10 & & 10 & 10 & 10 & 10 & 10 & 10 & 10 \\
\hline PU-HW.C5 & 15 & 9.7 & 7 & 15 & 7 & 5.6 & 3.5 & 10.8 & & 7 & & 7 & 7 & 15 & 15 & 14.4 & 1.2 & 9.1 \\
\hline PU-HW.C6 & 0 & - & - & - & - & - & - & - & & - & & - & - & - & - & - & - & - \\
\hline PU-HW.C7 & 5 & 3 & 3 & 2 & 2 & 4 & 1 & 2 & & 3 & & 2 & 1 & 3 & 3 & 3 & 3 & 2 \\
\hline PV-ALL.C1 & 50 & 0.5 & 0.5 & 0 & 30 & 0 & 0 & 0 & $\cdots$ & 30 & & 50 & 0 & 0 & 4.5 & 3.5 & 0 & 0 \\
\hline PV-ALL.C2 & 10 & 10 & 0 & 10 & 10 & 0 & 0 & 10 & & 0 & & 10 & 0 & 10 & 10 & 10 & 10 & 10 \\
\hline PV-ALL.C3 & 0 & - & - & - & - & - & - & - & & - & & - & - & - & - & - & - & - \\
\hline PV-ALL.C4 & 40 & 7.5 & 7.5 & 10 & 22.5 & 5 & 5 & 5 & & 32.5 & & 36.5 & 5 & 1 & 8.5 & 23 & 0 & 13 \\
\hline EV-HWBT.C1 & 30 & 30 & 10 & 20 & 5 & 5 & 0 & 5 & & 20 & & 10 & 5 & 30 & 20 & 30 & 5 & 10 \\
\hline EV-HWBT.C2 & 20 & 13 & 4 & 15 & 6 & 0 & 2 & 6 & & 14 & & 3 & 3 & 18 & 7 & 14 & 7 & 13 \\
\hline EV-HWBT.C3 & 30 & 17 & 9 & 25 & 27 & 0 & 0 & 0 & & 21 & & 4 & 4 & 4 & 4 & 4 & 4 & 10 \\
\hline EV-HWBT.C4 & 20 & 15 & 15 & 20 & 15 & 20 & 15 & 15 & & 15 & & 15 & 20 & 15 & 20 & 15 & 15 & 20 \\
\hline
\end{tabular}

${ }^{9}$ Some assessments are from the long-range plan of the metropolitan/transportation planning organization (MPO/TPO) (HRTPO, 2011b). 
Table 4.4-4 The baseline scores and rankings of a sample of the 93 candidate regional highway projects in the case study $4.4^{10}$.

\begin{tabular}{|c|c|c|}
\hline \multirow{2}{*}{ Projects } & \multicolumn{2}{|c|}{ Baseline scenario } \\
\hline & Scores & Ranks \\
\hline$p_{67}$ & 191.26 & 1 \\
\hline$p_{34}$ & 179.48 & 2 \\
\hline$p_{21}$ & 172.00 & 3 \\
\hline$p_{31}$ & 166.24 & 4 \\
\hline$p_{35}$ & 159.09 & 5 \\
\hline$p_{27}$ & 156.56 & 6 \\
\hline$p_{82}$ & 155.46 & 7 \\
\hline$p_{33}$ & 154.00 & 8 \\
\hline$p_{32}$ & 141.08 & 9 \\
\hline$p_{92}$ & 140.60 & 10 \\
\hline$\ldots$ & $\cdots$ & $\cdots$ \\
\hline$p_{61}$ & 127.28 & 18 \\
\hline$\ldots$ & $\cdots$ & $\cdots$ \\
\hline$p_{37}$ & 59.23 & 84 \\
\hline$p_{40}$ & 58.91 & 85 \\
\hline$p_{1}$ & 58.67 & 86 \\
\hline$p_{74}$ & 55.69 & 87 \\
\hline$p_{16}$ & 55.21 & 88 \\
\hline$p_{58}$ & 55.07 & 89 \\
\hline$p_{9}$ & 49.58 & 90 \\
\hline$p_{59}$ & 47.87 & 91 \\
\hline$p_{80}$ & 46.54 & 92 \\
\hline$p_{83}$ & 46.03 & 93 \\
\hline
\end{tabular}

${ }^{10}$ The scores are obtained via Eq. (2) and verified by the results in the long range plan of the MPO/TPO (HRTPO, 2011a). 


\subsubsection{Defining and incorporating scenarios of deep uncertainties}

Table 5 describes a set of emergent/future conditions and the construction of scenarios that combine the conditions. We identified the conditions by reviewing technical reports and transportation plans published by HRTPO and HRPDC published between 2008 and 2011. Figure 3 describes the frequency of particular conditions being mentioned in the approximately eight documents (HRPDC 2010; HRPDC 2011; EPA 2009; FHWA 2011; GCCC, 2008; Pyke et al., 2008; McAllister et al., 2008; IAW, 2009; U.S. GCRP, 2009). For the purpose of this case study, we assembled five scenarios from the most frequently mentioned conditions, as follows:

- Scenario $s_{1}$ : Climate change manifests via projected average sea-level rise (incorporated vertical changes in land subsidence or uplift) and increased storm surge levels (HRPDC, 2011) over the time horizon of the long range transportation plan;

- Scenario $s_{2}$ : All of scenario $s_{1}$ with the addition of a national-wide extended economic recession, which results in significant reduction of available funding;

- Scenario $s_{3}$ : All of scenario $s_{1}$ with the addition of accelerated wear and tear on the transportation facilities because of more extreme heat days and other related processes;

- Scenario $s_{4}$ : All of scenario $s_{1}$ with the addition of an adverse phenomena for ecosystems, and considering that land-use and other regulations will change dramatically to protect the ecosystems;

- Scenario $s_{5}$ : All of scenario $s_{1}$ with the addition of significant increase in traffic demand due to the growth of regional tourism, freight, and military logistics activities.

Table 6 provides the natural-language statements that we elicited with HRTPO staff on the change of criteria importance for each scenario $s_{1}$ through $s_{5}$, relative to the baseline scenario 
$s_{0}$. For example, under the scenario $s_{1}$, we elicited that safety and security has a Major Increase in importance relative to the baseline scenario. The other criteria stay the same in relative importance. Typically, a scenario requires no, one, or only a few criteria adjustments relative to the baseline scenario. When a scenario requires many adjustments (i.e., tends to change the decision maker's preference structure radically), re-eliciting all of the criteria weights for that scenario may be considered (Karvetski et al. 2011a). Note that although scenarios $s_{1}$ through $s_{5}$ share the identical climate conditions, the elicitation emphasized the effect of system emergence and the offsetting between conditions, so that the elicited statements on criteria importance change for scenario $s_{1}$ is not a subset of those in scenario $s_{2}$ through $s_{5}$.

We used the statements elicited above to adjust the weights per scenario according to the approach described in section 2.2. In this case study, the multipliers in Eq. (3) were defined as $\delta=5, \varepsilon=3$ according to the engagement of stakeholders and experts in workshops and brainstorming sessions. Schroeder et al. (2010) explore the validity and sensitivity analysis on the values of these multipliers. Table 7 provides the new weights for each scenario. We re-scored and re-ranked the 93 projects for each scenario using the new criteria weights along with the HRTPO's assessments of the projects across the criteria. The results are interpreted below. The case study has suggested the efficiency of the approach to identify and assess the impacts of new scenarios, avoiding onerous elicitation of stakeholders. We needed only ten adjustments (Table 6) to update the baseline value function across all of the additional five scenarios. Completely reweighting the criteria with the swing weight technique for the five scenarios would require at least $14 * 5=70$ total ratio comparisons. Such a tedious approach might be warranted once the scenarios are refined and the funding decisions become contentious or imminent (Karvetski et al. 2011b). 

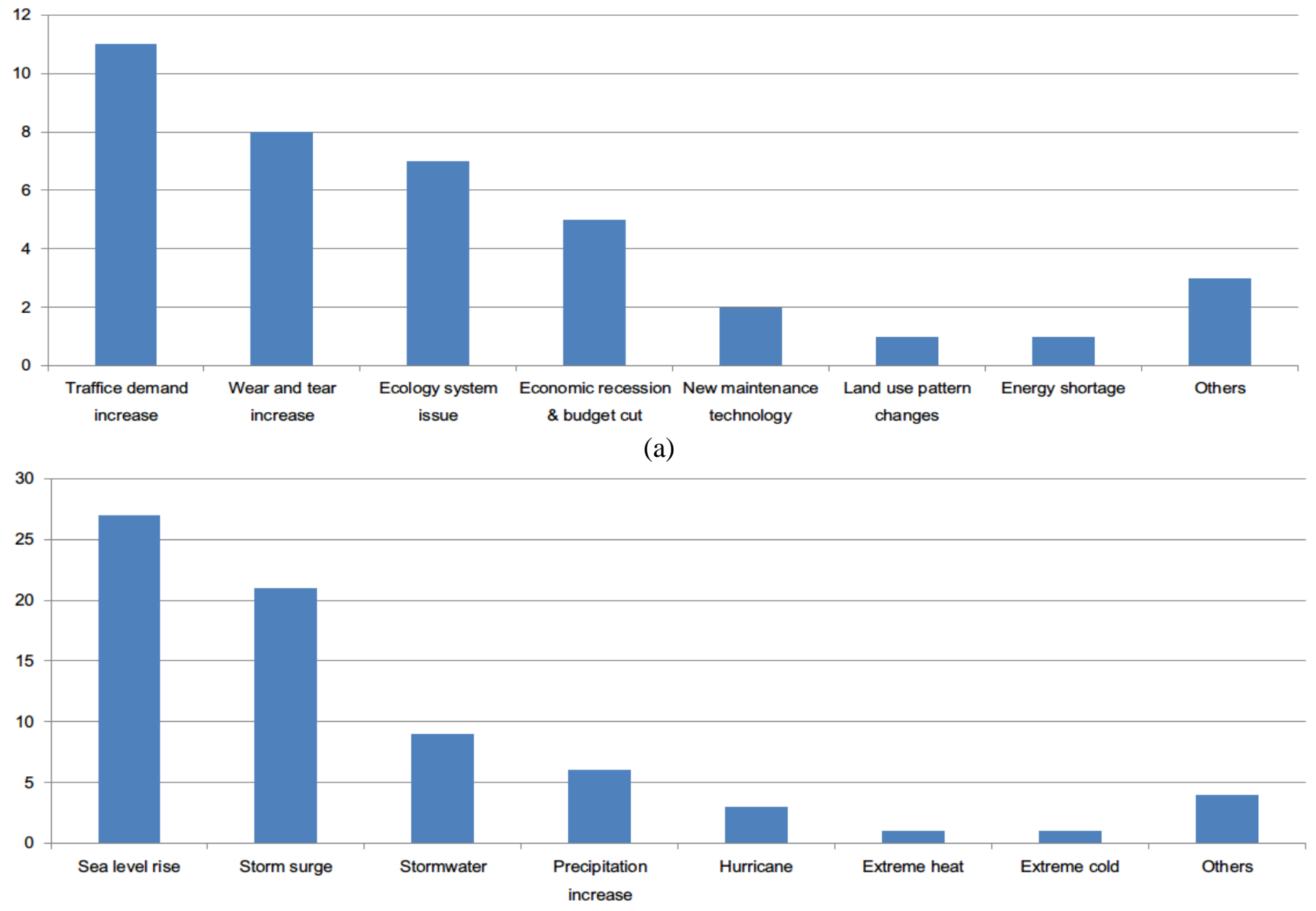

(b)

Figure 4.4-1 Frequencies of conditions being mentioned in science study assessment reviewed in the case study 4.4: (a) climate conditions (b) non-climate conditions 
Table 4.4-5 The identified conditions and scenario definition in the case study 4.4.

\begin{tabular}{|c|c|c|c|c|c|}
\hline \multirow{2}{*}{ Conditions } & \multicolumn{5}{|c|}{ Scenarios } \\
\hline & $s_{1}$ & $s_{2}$ & $s_{3}$ & $s_{4}$ & $s_{5}$ \\
\hline Increase in sea level rise & $\mathrm{x}$ & $\mathrm{x}$ & $\mathrm{X}$ & $\mathrm{X}$ & $\mathrm{x}$ \\
\hline Increase in storm surge & $\mathrm{x}$ & $\mathrm{x}$ & $\mathrm{x}$ & $\mathrm{X}$ & $\mathrm{x}$ \\
\hline Increase in precipitation & & & & & \\
\hline Increase in stormwater & & & & & \\
\hline Increase in storm frequency & & & & & \\
\hline Increase in days below freezing & & & & & \\
\hline Increase in extreme heat days & & & & & \\
\hline Increased occurrence of drought & & & & & \\
\hline Economic recession & & $\mathrm{x}$ & & & \\
\hline No further increase on federal government debt cap & & & & & \\
\hline Increased wear and tear on public infrastructure & & & $\mathrm{x}$ & & \\
\hline New technology for maintenance / inspection & & & & & \\
\hline Increase in traffic demand & & & & & $\mathrm{x}$ \\
\hline Increase in area tourism & & & & & $\mathrm{x}$ \\
\hline Population growth & & & & & \\
\hline Energy shortage & & & & & \\
\hline Changes in land use regulation & & & & & \\
\hline Increased infectious disease occurrence & & & & $\mathrm{X}$ & \\
\hline Increased loss of forest and plant life & & & & $\mathrm{X}$ & \\
\hline Increased mortality of native animal species & & & & $\mathrm{x}$ & \\
\hline
\end{tabular}


Table 4.4-6 The natural-language statements regarding the changes in relative importance for the criteria for each scenario $s_{1}$ to $s_{5}$ in the case study 4.4 .

\begin{tabular}{|c|c|c|c|c|c|}
\hline \multirow[b]{2}{*}{ Criteria } & \multicolumn{5}{|c|}{ Scenarios } \\
\hline & $\begin{array}{c}s_{1} \\
\text { Climate change only }\end{array}$ & $\begin{array}{c}s_{2} \\
{\left[s_{1}+\text { Economic }\right.} \\
\text { recession }]\end{array}$ & $\begin{array}{c}s_{3} \\
{\left[s_{1}+\text { Wear and tear }\right]}\end{array}$ & $\begin{array}{c}s_{4} \\
{\left[s_{l}+\text { Ecosystem stress }\right]}\end{array}$ & $\begin{array}{c}s_{5} \\
{\left[s_{1}+\text { Traffic demand }\right]}\end{array}$ \\
\hline PU-HW.C1 & & & & & MAJOR INCREASE \\
\hline PU-HW.C2 & & & & & \\
\hline PU-HW.C3 & & MAJOR INCREASE & & & \\
\hline PU-HW.C4 & & & & MAJOR INCREASE & \\
\hline PU-HW.C5 & MAJOR INCREASE & & MAJOR INCREASE & & minor increase \\
\hline PU-HW.C6 & minor increase & & MAJOR INCREASE & & \\
\hline PU-HW.C7 & & & & & \\
\hline PV-ALL.C1 & & & & & \\
\hline PV-ALL.C2 & & & & & \\
\hline PV-ALL.C3 & & & & & \\
\hline PV-ALL.C4 & & & & & \\
\hline EV-HWBT.C1 & & & & & MAJOR INCREASE \\
\hline EV-HWBT.C2 & & & & & \\
\hline EV-HWBT.C3 & & MAJOR INCREASE & & & \\
\hline EV-HWBT.C4 & & minor increase & & & \\
\hline
\end{tabular}

* We elicited MAJOR DECREASE and minor decrease in addition to MAJOR INCREASE and minor increase in other of our efforts cited in the paper narrative. We did not encounter "decrease" in elicitations performed for the current effort, despite that we encouraged/allowed this response with the stakeholders/experts. 
Table 4.4-7 The adjusted weights of the criteria for the scenarios $s_{1}$ to $s_{5}$ in the case study 4.4.

\begin{tabular}{|c|c|c|c|c|c|}
\hline \multirow{3}{*}{ Criteria } & \multicolumn{5}{|c|}{ Scenarios } \\
\hline & $s_{1}$ & $s_{2}$ & $s_{3}$ & $s_{4}$ & $s_{5}$ \\
\hline & Climate change only & {$\left[s_{1}+\right.$ Economic recession $]$} & {$\left[s_{1}+\right.$ Wear and tear $]$} & {$\left[s_{l}+\right.$ Ecosystem stress $]$} & {$\left[s_{1}+\right.$ Traffic demand $]$} \\
\hline PU-HW.C1 & 0.188 & 0.188 & 0.253 & 0.214 & 0.629 \\
\hline PU-HW.C2 & 0.156 & 0.156 & 0.211 & 0.179 & 0.105 \\
\hline PU-HW.C3 & 0.094 & 0.469 & 0.127 & 0.107 & 0.063 \\
\hline PU-HW.C4 & 0.063 & 0.063 & 0.084 & 0.357 & 0.042 \\
\hline PU-HW.C5 & 0.469 & 0.094 & 0.283 & 0.107 & 0.141 \\
\hline PU-HW.C6 & 0.000 & 0.000 & 0.000 & 0.000 & 0.000 \\
\hline PU-HW.C7 & 0.031 & 0.031 & 0.042 & 0.036 & 0.021 \\
\hline PV-ALL.C1 & 0.500 & 0.500 & 0.500 & 0.500 & 0.500 \\
\hline PV-ALL.C2 & 0.100 & 0.100 & 0.100 & 0.100 & 0.100 \\
\hline PV-ALL.C3 & 0.000 & 0.000 & 0.000 & 0.000 & 0.000 \\
\hline PV-ALL.C4 & 0.400 & 0.400 & 0.400 & 0.400 & 0.400 \\
\hline EV-HWBT.C1 & 0.300 & 0.123 & 0.300 & 0.300 & 0.682 \\
\hline EV-HWBT.C2 & 0.200 & 0.082 & 0.200 & 0.200 & 0.091 \\
\hline EV-HWBT.C3 & 0.300 & 0.613 & 0.300 & 0.300 & 0.136 \\
\hline EV-HWBT.C4 & 0.200 & 0.183 & 0.200 & 0.200 & 0.091 \\
\hline
\end{tabular}




\subsubsection{Results}

Table 4.4-8 describes the implications of climate change combining with several other conditions, in terms of the re-scoring and the re-ranking of the projects per scenario. We obtained the scores and rankings for scenarios $s_{1}$ to $s_{5}$, through the incremental adjustment of the value functions as described above. For example, project $p_{86}-$ Wythe Creek Road scores a 166.24 in the baseline scenario $s_{0}$, a 170.01 in scenario $s_{1}$-climate change only scenario, and a 144.84 in scenario $s_{5}$-climate change combining with increased traffic demand. These different scores reflect the impact of scenarios on the overall assessment of the project.

Figure 4.4-2 describes the range of the project scores across the scenarios. Figure 4.4-3 describes the range of the project rankings (priority order) across the scenarios. The ordinal information of Figure 4.4-3 is a "lossy" summary of the corresponding cardinal information in Figure 4.4-2. However, the ordinal rankings could be more effective than the cardinal scores to distinguish projects for funding priority. In the two figures, each diamond represents a project in the baseline scenario. The maxima and minima, of score or ranking, across the other scenarios are represented by the vertical bars extending above and below the diamonds. A taller bar suggests a greater influence of scenarios on the priority of the project. The representation provides insight to where the scenarios increase the upside or downside potential of the project (Karvetski et al. 2011c). Inspection of these figures typically leads the analyst and stakeholders to iterate on the input assumptions and intermediate steps of the analysis. For example, the bar associated with the project $p_{61}-$ Dam Neck Road shows that this project is ranked at 18 under baseline scenario. The selection of this project is very robust to the 5 additional climate change scenarios, because the lowest ranking of this project is 20 . On the other hand, this project has potential to be critical to stakeholders in the scenarios $s_{5}$ - climate change combining with 
increased traffic demand. If stakeholders are curious about the reason for these characteristics of this project, Table 4.4-3 can be investigated to obtain more understanding about this project. It can be easily recognized that the changes in the ranking of the project $p_{61}$-Dam Neck Road through scenarios are largely dominated by the importance of the criterion PU-HW.Cl Congestion Level, because the project is assessed to be full score on the criterion PU-HW.Cl and average scores on other criteria.

Table 4.4-9 describes how projects can score high/low in the baseline and yet have relatively low/high sensitivity to scenarios including climate change, etc. The stratification of the baseline priority ranking is defined by simple clustering analysis on the baseline scores. We defined a high baseline priority ranking for projects ranked 1-15, and a low baseline priority ranking for projects ranked 66-97. We defined a large increase in ranking as an increase in priority by five or more positions and a large decrease in ranking as a decrease in priority by five or more positions. The table shows that project $p_{32}-$ Turnpike Road is ranked as high priority in baseline, however has a large potential decrease in ranking due to one or more scenarios. Such a result suggests that planning for this project should focus on the disruptive scenarios. Project $p_{21}$ - Middle Ground Blvd, $p_{34}$ - Holland Road and $p_{67}$ - Lynnhaven Pkwy (Phase 2) are high priority projects with rankings that are stable across scenarios. These three projects are top candidates for investment regardless of the climate and climate-included scenarios. Project $p_{4}-$ Route 17 (G.W. Memorial Highway) and project $p_{65}$ - London Bridge Road are unique among the projects to be ranked at low priority and have large increases in priority across scenariosthe scenarios present these two projects opportunity for funding.

Next we characterized which scenarios most influenced the prioritizations. We ordered the scenarios according to the metric defined above by Eq. (7). Table 4.4-10 describes the 
scenario-influence metric for the five scenarios. The most influential scenario according to this metric is scenario $s_{5}-$ Climate change combined with increased traffic demand. The scenario $s_{4}$ - Climate change combined with degradation of ecosystems changes the baseline ranking order the least. As the analysis is re-visited every four years, the influential and non-influential scenarios should be compared to the corresponding scenarios in previous planning cycles. 
Table 4.4-8 The scores and rankings of a sample of the 93 candidate regional highway projects across five scenarios in the case study 4.4.

\begin{tabular}{|c|c|c|c|c|c|c|c|c|c|c|}
\hline \multirow{3}{*}{ Projects } & \multicolumn{10}{|c|}{ Scenarios } \\
\hline & \multicolumn{2}{|c|}{$s_{1}}$. & \multicolumn{2}{|c|}{$s_{2}$. } & \multicolumn{2}{|c|}{$s_{3}$. } & \multicolumn{2}{|c|}{$s_{4}$. } & \multicolumn{2}{|c|}{$s_{5}$} \\
\hline & Score & Rank & Score & Rank & Score & Rank & Score & Rank & Score & Rank \\
\hline$p_{67}$ & 185.7 & 1 & 197.5 & 1 & 188.9 & 1 & 202.0 & 1 & 185.0 & 1 \\
\hline$p_{34}$ & 178.8 & 2 & 185.4 & 2 & 179.2 & 2 & 186.6 & 2 & 148.9 & 3 \\
\hline$p_{21}$ & 164.6 & 4 & 154.5 & 7 & 168.9 & 3 & 184.9 & 3 & 179.3 & 2 \\
\hline$p_{31}$ & 170.0 & 3 & 166.0 & 3 & 167.8 & 4 & 176.9 & 4 & 144.8 & 4 \\
\hline$p_{35}$ & 163.4 & 5 & 161.6 & 4 & 160.9 & 5 & 169.9 & 6 & 136.0 & 9 \\
\hline$p_{27}$ & 148.2 & 6 & 143.7 & 11 & 153.1 & 6 & 165.4 & 7 & 143.0 & 6 \\
\hline$p_{82}$ & 145.1 & 8 & 148.7 & 8 & 151.1 & 7 & 173.0 & 5 & 142.4 & 7 \\
\hline$p_{33}$ & 145.3 & 7 & 159.3 & 5 & 150.4 & 8 & 162.6 & 8 & 133.4 & 10 \\
\hline$p_{32}$ & 130.4 & 13 & 157.1 & 6 & 136.6 & 10 & 158.9 & 9 & 118.6 & 15 \\
\hline$p_{92}$ & 130.8 & 12 & 128.3 & 15 & 136.5 & 11 & 146.4 & 13 & 127.5 & 12 \\
\hline$\ldots$ & $\ldots$ & & & & & & & & & \\
\hline$p_{61}$ & 127.4 & 16 & 128.7 & 16 & 134.1 & 13 & 132.0 & 20 & 144.7 & 5 \\
\hline$\ldots$ & $\ldots$ & & & & & & & & & \\
\hline$p_{37}$ & 63.4 & 74 & 60.4 & 79 & 61.0 & 82 & 75.7 & 85 & 39.9 & 84 \\
\hline$p_{40}$ & 63.2 & 75 & 63.2 & 77 & 60.7 & 84 & 75.5 & 87 & 37.3 & 87 \\
\hline$p_{1}$ & 62.0 & 78 & 43.2 & 87 & 60.0 & 86 & 79.1 & 80 & 41.7 & 81 \\
\hline$p_{74}$ & 43.1 & 92 & 67.5 & 71 & 50.4 & 91 & 74.6 & 88 & 35.7 & 89 \\
\hline$p_{16}$ & 62.8 & 76 & 37.3 & 90 & 58.4 & 87 & 74.3 & 89 & 47.2 & 74 \\
\hline$p_{58}$ & 53.3 & 90 & 42.1 & 89 & 54.3 & 88 & 75.6 & 86 & 38.6 & 85 \\
\hline$p_{9}$ & 57.4 & 85 & 35.3 & 91 & 52.8 & 89 & 68.8 & 90 & 37.2 & 88 \\
\hline$p_{59}$ & 55.5 & 86 & 30.6 & 93 & 51.0 & 90 & 68.5 & 91 & 34.3 & 91 \\
\hline$p_{80}$ & 41.1 & 93 & 42.7 & 88 & 44.3 & 93 & 66.7 & 92 & 26.6 & 92 \\
\hline$p_{83}$ & 55.1 & 88 & 32.7 & 92 & 49.8 & 92 & 66.3 & 93 & 25.4 & 93 \\
\hline
\end{tabular}




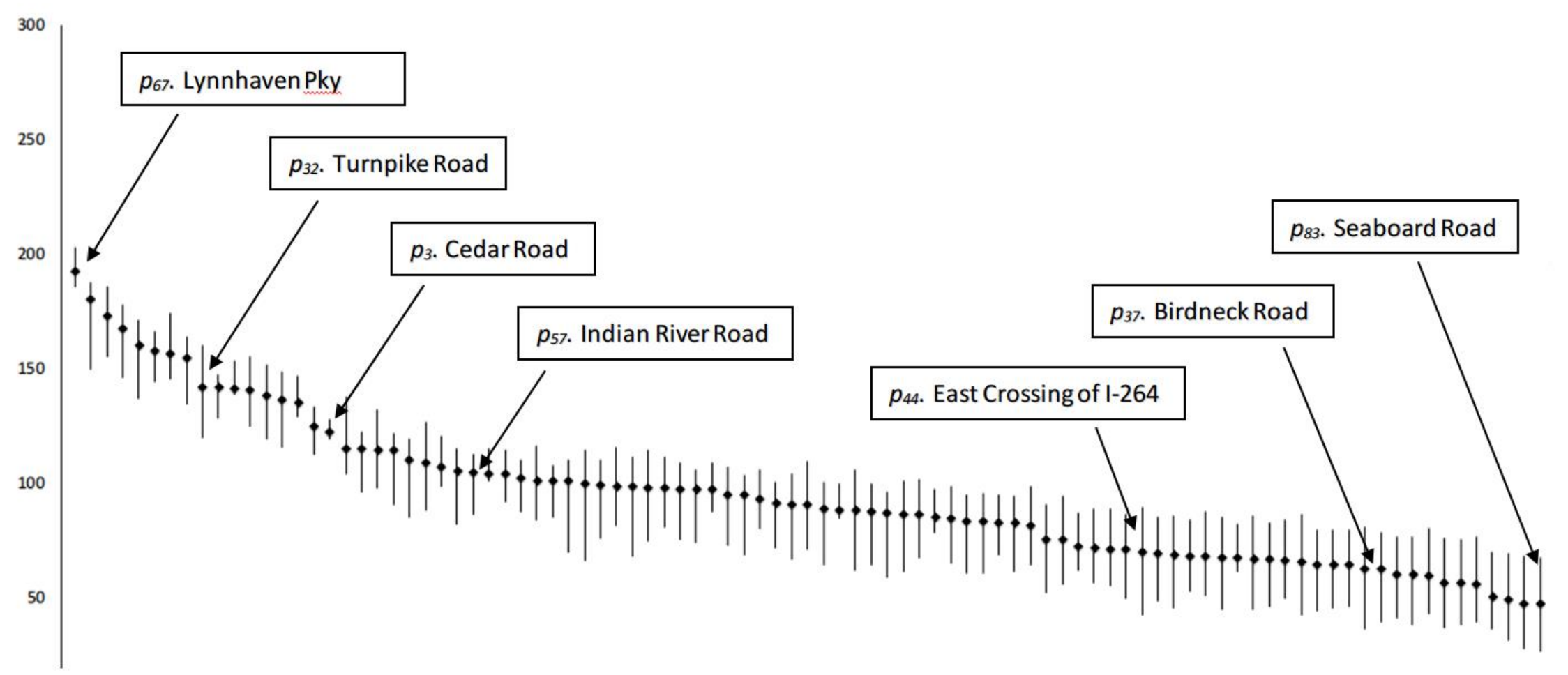

Figure 4.4-2 The baseline scores (diamonds) and the ranges (vertical bars) of scores associated to the five scenarios of the 93 candidate regional highway projects addressed in the case study 4.4 


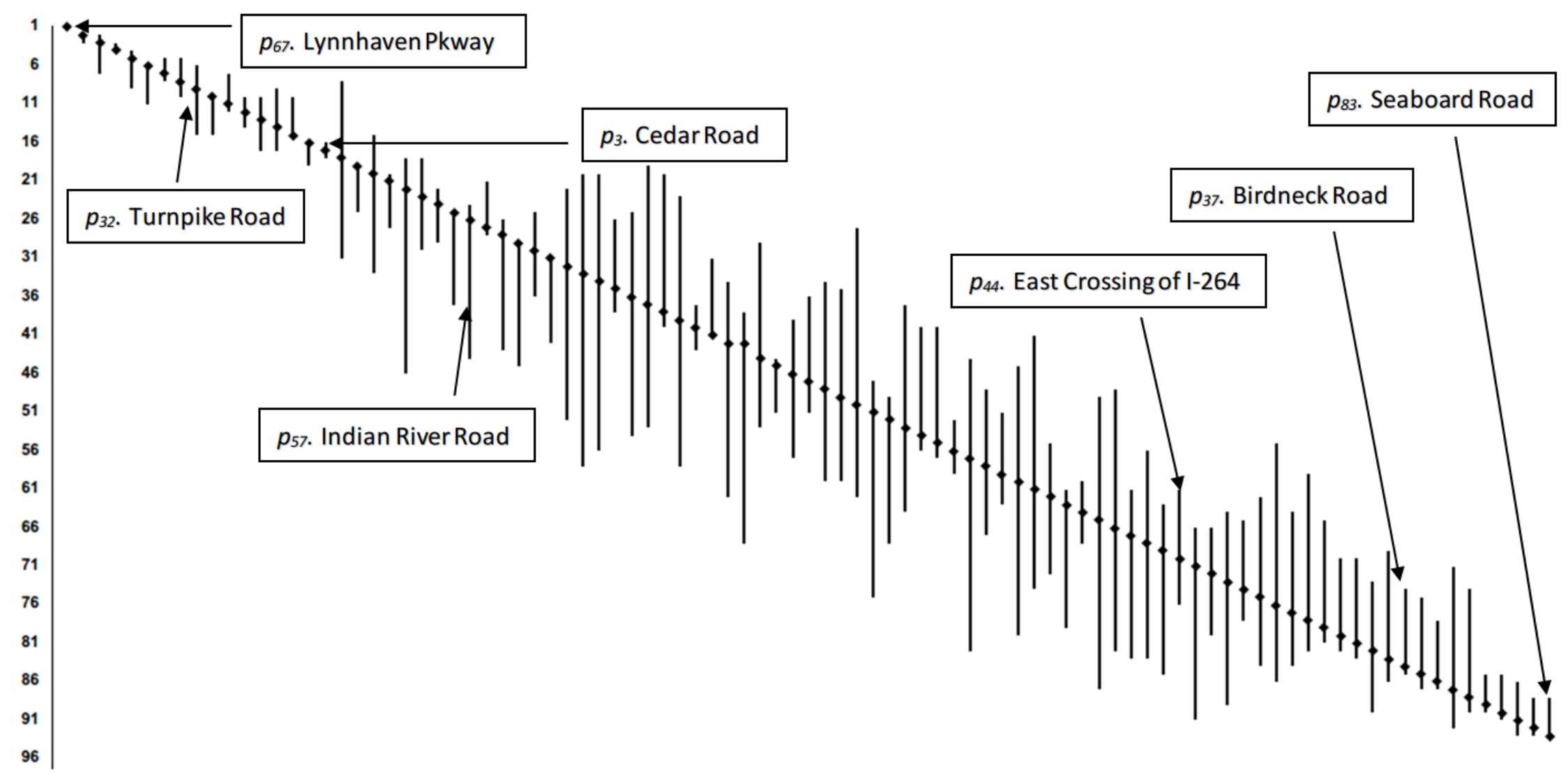

Figure 4.4-3 The baseline rankings (diamonds) and the ranges (vertical bars) of scores associated to the five scenarios of the 93 candidate regional highway projects addressed in the case study 4.4 
Table 4.4-9 A characterization of the projects in terms of baseline priority ranking and changes in ranking induced by the scenarios in the case study 4.4

\begin{tabular}{lll}
\hline & High baseline priority ranking & \multicolumn{1}{c}{ Low baseline priority ranking } \\
\hline $\begin{array}{l}\text { Large increase in } \\
\text { priority }\end{array}$ & $p_{6}-$ Mt. Pleasant Road, Phase 1 & $p_{4}-$ Route 17 (G.W. Memorial Highway) \\
& $p_{23}-$ Route 60 Relocated & $p_{65}-$ London Bridge Road \\
\hline $\begin{array}{l}\text { Stable } \\
\text { Performance }\end{array}$ & $p_{21}-$ Middle Ground Blvd & $p_{58}-$ Mt. Pleasant Road, Phase 1 \\
& $p_{34}-$ Holland Road (Route 58) & $p_{80}-$ Salem, Phase 2 \\
\hline \multirow{2}{*}{$\begin{array}{l}\text { Large decrease in } \\
\text { priority }\end{array}$} & $p_{27}-$ - Military Hwy & \\
& $p_{32}-$ Turnpike Road & $p_{52}-$ General Booth Blvd, Phase 3 \\
& $p_{92}-$ Witchduck Road & $p_{78}-$ Salem Road, Phase 1 \\
\hline
\end{tabular}

Table 4.4-10 The $\phi$ value quantifying the influence of scenarios, describing the influences of scenarios $s_{2}$ and $s_{5}$ are relatively greater than influence of other scenarios in the case study 4.4.

\begin{tabular}{ll}
\hline Scenarios & $\phi$ Value \\
\hline$s_{1}$. Climate change in terms of projected sea-level rise and increased storm surge levels & 0.018 \\
$s_{2}$. All conditions in scenario s1 with the addition of an economic recession & 0.046 \\
$s_{3}$. All conditions in scenario s1 with the addition of accelerated wear and tear & 0.023 \\
$s_{4}$. All conditions in scenario s1 with the addition of adverse phenomena for ecosystems & 0.016 \\
$s_{5}$. All conditions in scenario s1 with the addition of significant increased traffic demand & 0.052 \\
\hline
\end{tabular}

* Larger values of $\phi$ indicate greater influence of the scenarios, where $0<\phi<1$. 


\subsection{Case Study: VTrans 2035 Multimodal Policies}

\subsubsection{Overview}

This case study is a scenario based analysis of the VTrans 2035, which is Virginia's statewide long-range multimodal policy plan. The objectives of this plan are establishing the vision, goals and investment priorities for the Virginia Commonwealth's public transportation systems. As climate change manifests itself and more social / economical changes have been observed, the robustness of this plan becomes a concern of planning organizations. We applied the introduced analytical framework to test the performance and robustness of this plan, with cooperation from a regional Metropolitan Planning Organization.

The inputs for the variables in the analytical frameworks are obtained from on-site stakeholder meetings. The attendees of the meetings include intermodal planning experts from the Virginia Department of Transportation (VDOTs), transportation regional planners from HRTPO, and faculty members and research assistants of University of Virginia.

\subsubsection{Identify policies and evaluation criteria}

As above, the initial steps of the analytical framework include identifying policies for evaluation, defining policy evaluation criteria and assemble scenarios from emergent conditions. Table 4.5-1 displays the total twenty-five identified policies. The list of policies in the scope includes policies which were identified in a statewide long-range transportation plan (VDOT, 2004) and four region-specific policies. Examples of the policies include increasing rail funding, improving connections between transportation modes, and starting a trust fund for transportation. 
We continued to use the policy portfolio assessment criteria from the long-range transportation plan (VDOT, 2004) as the performance measurement of policies under emergent scenarios. Table 4.5-2 provides a sample of these criteria and sub-criteria (there are 6 criteria, 13 sub-criteria and 34 sub-sub criteria included in the case study.). The performance criteria covers planning topics include efficiency, safety, feasibility, economic vitality, and quality of life. Subcriteria and even sub-sub criteria were defined to elaborate the criteria by incorporating qualitatively measurable metrics or breaking it down to specifically and unambiguously defined questions. For example, the sub-criterion C.1.1.a under safety asks whether a policy will, "improve safety for system users and operators within the system and at mode origins/destinations". The corresponding sub-sub criteria could be improving safety at at-grade crossings, improving bicycle and pedestrian safety, correct sub-standard (safety) designs and other geometric/pathway (e.g., runway obstructions, channel depth, bridge clearance, etc) deficiencies). 
Table 4.5-1 The polices needing evaluation through scenario-based analysis in the case study 4.5

P.1 - Invest more in transportation

P.2 - Support Transit

P.3 - Remove Bias

P.4 - Fund Rail

P.5 - Protect Trust Fund Rev. for Trans.

P.6 - Strengthen planning and modeling

P.7 - Manage Access

P.8 - Consider State vs. Local Rules

P.9 - Address Transportation/Land Use Conflicts

P.10 - Improve Connections

P.11 - Think Multimodally

P.12 - Take the Lead

P.13 - Invest in Technology

P.14 - Use Objective Criteria

P.15 - Plan Multimodally

P.16 - Continue Public and Stakeholder Involvement

P.17 - Continue Transportation Agency Head Coord.

P.18 - Review Intermodal Office Alignment

P.19 - Develop Action Plans

P.20 - Continue Technical Committee

P.21 - Establish a Commission

P.22 - Smart Growth

P.23 - Bicycle and Pedestrian Facilities Feeder System

P.24 - Going Green

P.25 - Diesel and Filter Regulation 
Table 4.5-2 Sample of the Policy Evaluation Criteria in the case study 4.5

Criterion 1: Provide a safe, secure, and integrated transportation system that reflects different needs of the commonwealth.

\begin{tabular}{|c|c|}
\hline 1.1 Safety & 1.1.a Reduction in crashes and/or incidents \\
\hline & $\begin{array}{l}\text { 1.2. a Reduction in security breaches or losses because of theft, } \\
\text { vandalism, or other incidents }\end{array}$ \\
\hline 1.2 Security & $\begin{array}{l}\text { 1.2.b Ability to meet strategic and emergency transportation needs; } \\
\text { ability to perform in the event of an attack or natural disaster }\end{array}$ \\
\hline
\end{tabular}

Criterion 2: Preserve and manage the existing transportation system through technology and more efficient operations.

2.1 Preservation
2.2 Management
need addressed; bridge condition
2.2. a Consistency with local comprehensive plans,
metropolitan planning organization (MPO) plans,
or other regional plans; number of access breaks
$2.2 . \mathrm{b}$ Tons of freight moved; number of people
moved; ease of transition to new technology
$2.2 . \mathrm{c}$ Reduction in time to clear nonrecurring events; on-time
performance of system and services; reduction in travel time
variability; reduction in unexpected delay
2.2. d Reduction in transfer time

Criterion 3: Facilitate the efficient movement of people and goods, expand choices, and improve interconnectivity of all transportation modes.

3.1 Mobility for all

3.2 Accessibility for all

3.3 System connectivity 3.1.a Reduction in vehicle miles traveled; level of service improvement; reduction in travel delay

3.1.b Number of mode choices provided; number of alternatives to highway travel

3.1.c Increase in system capacity

3.2. a Number of modes serving activity center; frequency of service to activity center

3.2.b Number of mode choices provided; service to special needs populations

3.3.a Number of barriers removed; number of links added; increase in the number of modal connections; number of bus turnouts, park-andride spaces, and bicycle/pedestrian accommodations

Criterion 4: Improve Virginia's economic vitality and facilitate the coordination of transportation, land use, and economic development planning activities.

4.1 Statewide economic vitality

4.2 Consistency with local and regional goals
4.1. a Number of mode choices; proximity of service or facility to desired destination, unemployment rate 4.1.b Number of modes serving market; travel time; travel cost

4.1.c Number of mode choices; proximity of service or facility to desired destination

4.2.b Consistency with local comprehensive plans, MPO plans, or other regional plans; consistency with local zoning and land uses; consistency with local economic development goals 


\subsubsection{Defining the scenarios}

Twenty scenarios have been identified via public involvement, scenario-planning workshop, and document analysis on existing technical report or vision roadmaps. Table 4.5-3 shows the completed list of these scenarios. As the purpose of demonstration, analysis on five selected scenarios is presented in this case study. These scenarios are in five categories, demographic, spatial, economic, environmental, and others.

Scenario, S.2 - Urban sprawl: as future residential and commercial buildings are continuously planned and developed around the region while the number of sites to build in the city decreases, urban sprawl may increase. And urban sprawl is becoming an issue for many places of growth in Commonwealth of Virginia and the United States.

Scenario, S.17 - Retirement: aging population is now an important issue as the babyboomer generation is reaching the retirement age. An aging population "implies additional transit needs, changing housing needs, the need for heightened safety standards, and residents with inflexible financial situations" (US FHWA, 2007). As the demographics of an area change, the transportation system may need to adapt accordingly to meet new demands.

Scenario S.18 - Natural disasters relevant to the region: the hurricane Katrina and Sandy indicated the big threats of natural disaster to coastal areas. Through the study of historic records of disasters and local environmental / geographical characteristics, the hazard analysis workgroup of the region raised the significance of potential natural disaster include storm surge and flooding.

Scenario, S.3 - IT amenities growing in the region: in the past decade, a great number of companies have chosen to re-locate their facilities and headquarters to this areas due to the state 
and local policies that encourage business growth, a highly educated and skilled technology workforce, and expanded advanced IT infrastructure (Commonwealth of Virginia, Office of the Governor, 2007). This scenario is assembled to describe the future in which information and high-technology engineering industry develop in several surrounding areas.

Scenario, S.19 - Decrease in air quality (Christopulos, 2007): this scenario is composed to address the impacts of heavy intermodal freight transfer activities and the use of relevant facilities. Ten areas around the region had recently been selected as potential locations for a new rail and truck intermodal transfer station. These stations are believed to cause a decrease in air quality. 
Table 4.5-3 Emergent scenarios used for user reweighting of the criteria in the case study 4.5

\begin{tabular}{l}
\hline S.1 - Urban core repopulates \\
S.2 - Sprawl accelerates \\
S.3 - IT amenities grow \\
S.4 - Region undivided \\
S.5 - Transit oriented development \\
S.6 - Regional economy strengthens \\
S.7 - Global trade intensifies \\
S.8 - Energy cost rises \\
S.9 - Infrastructure investment expands \\
S.10 - In-migration increases \\
S.11 - Out-migration increases \\
S.12 - More households \\
S.13 - "Green" region emphasis \\
S.14 - Crisis of homeland security tightened \\
S.15 - Carbon constrained future \\
S.16 - Energy constrained future \\
S.17 - Retirement \\
S.18 - Natural disaster \\
S.19 - Decrease in air quality \\
S.20 - Sea Rise \\
\hline
\end{tabular}




\subsubsection{Baseline assessment}

A state-wide survey (Southeastern Institute of Research Inc., 2004) of 1,200 sampled state residents facilitated by the state department of transportation agreed that the policy evaluation criteria in the case study are roughly equally important ${ }^{11}$. After the review workshop with regional planning organizations, it is believed reasonable to assign equal weights to the criteria under the baseline scenario ("as-planned" scenario) based on the survey results.

Next, the baseline assessment needs to calculate or elicitation scores / assessments for each policy against the policy evaluation criteria, and determine the overall performance of policies via the additive value function. To determine these scores / assessments for policies across the evaluation criteria, we modelled or interviewed experts on the impact of each policy across each of the policy evaluation criteria. The additive value function for the analysis was consistent with the function used in Lambert et al. (2012) and You et al. (2012). Table 4.5-4 shows the baseline assessment results using a high, medium and low/unknown impact scale.

\subsubsection{Scenario analysis}

The critical task for the scenario analysis is to reweight policy evaluation criteria according to the changes on relative importance under the selected scenarios. To avoid unnecessary complexity, only the top level criteria (i.e. safety, land preservation, efficient movement of people and goods, economic vitality, quality of life, and program delivery) are

11 The survey showed that over $80 \%$ people rate these goals at least a ' 4 ' on a 5-point scale, where ' 5 ' is "very important and ' 1 ' is "not at all important." 
subjected to reweighting, which means if a transportation planner increases the weight for a criterion, the scores for all sub-criteria receives in the criterion will correspondingly increase under that scenario.

Table 4.5-5 shows the elicited inputs regarding the change in criterion importance in a scenario, from transportation planners and local experts that are interviewed. The inputs are described by two levels of natural language statements major increase to major decrease. In the result, we did not find that any criterion received major decreases in importance change. Most of the inputs from planners were minor increases and minor decreases. Major increases and major decreases modify the magnitude of the criteria weight by 8 times, minor decreases and minor increases modify the magnitude of the criteria weight by 6 times, according to the parameters defined in similar context in the additive weight function of the analytic hierarchical process (AHP) (Saaty, 1980) ${ }^{12}$. The changes in weight for the criteria are all relative, implying that if all criteria get same increase or decrease changes in weights for a scenario, the criteria will keep the same weight as in the as-planned scenario.

With the re-weighted criteria, we recalculate the overall scores of the policies via the additive value function. Tables are generated to display the scores of the policies under the scenarios, the ranking of each policy against other policies for each scenario. Figures are generated to visualize the range of policy sensitivities to the five scenarios.

${ }^{12}$ The parameters can be reset by the user with minimal effort via a software workbook. 
Table 4.5-4 Policy assessments against criteria in the case study $4.5^{13}$.

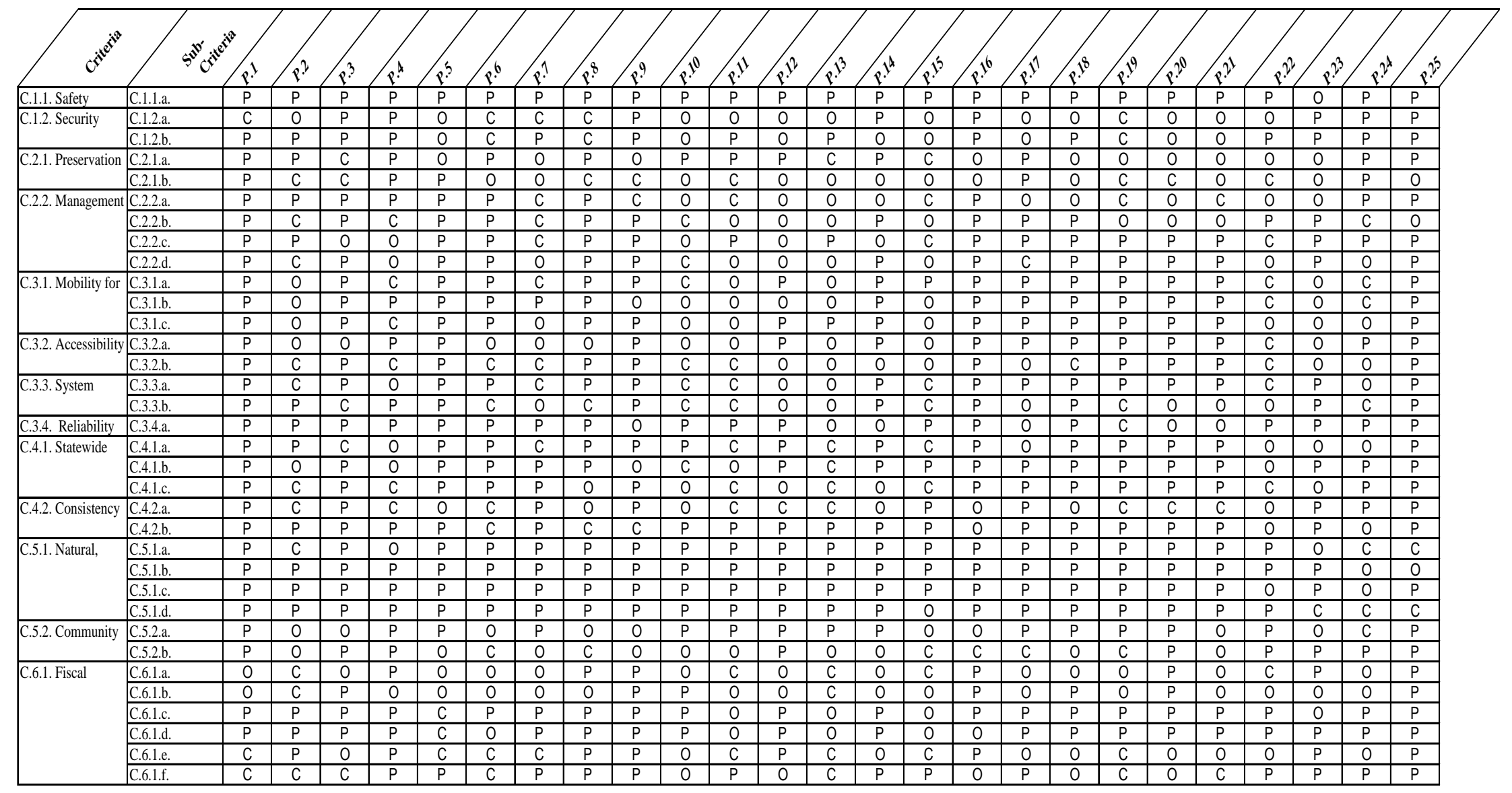

* $\mathrm{P}=$ minimal or unknown impact, $\mathrm{O}=$ moderate impact, $\mathrm{C}=$ high impact

${ }^{13}$ Table is adapted from the work of Schoroder and Lambert., 2012 
Table 4.5-5 Criteria reweighting results from scenario-based methodology in the case study 4.5.

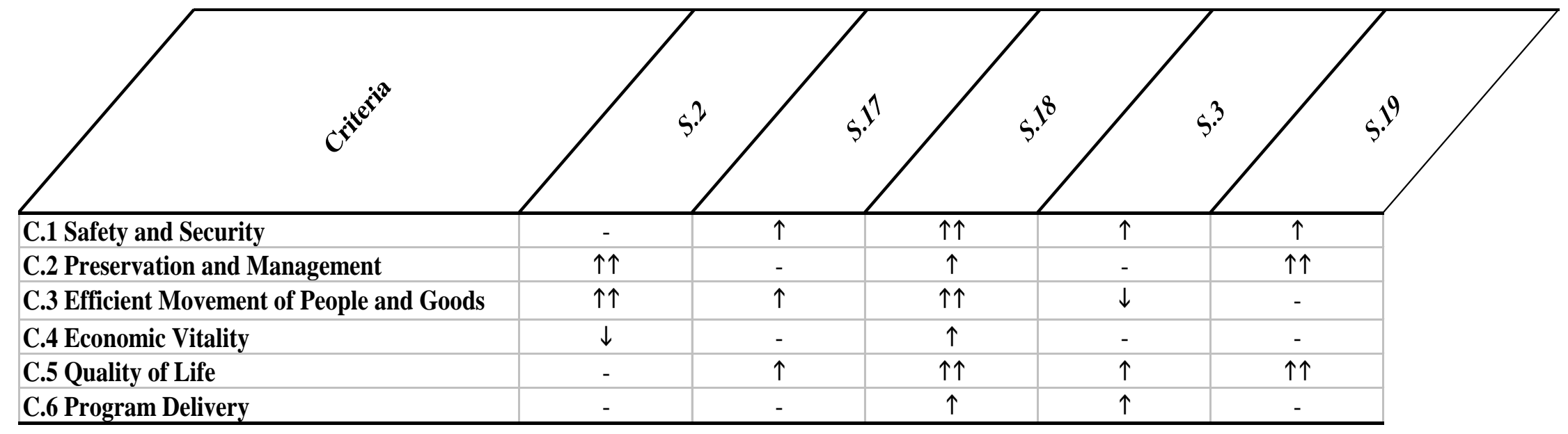

* Changes in importance $-=$ No change for the scenario, $\square \square=$ major increase, $\square=$ minor increase, $\square \square=$ major decrease, $\square=$ minor decrease 


\subsubsection{Results}

Table 4.5-6 shows the results of the policy scoring after incorporating scenario impacts. The policy scores under future scenarios are represented by the additive value function values resulting from the re-weighted policy evaluation criteria. The first scenario in Table 4.5-6, "Default Criteria Weights", is the criteria weights under the baseline scenario. The different scores of each policy under the rest of the future scenarios show the result after the criteria reweighting. For example, policy P.10 - Address Transportation/Land Use Conflicts scores '35' in the as-planned scenario, '43' in scenario S.2 - Sprawl Accelerates, '42' in scenario S.17 Retirement, '36' in scenario S.18 - Natural Disaster, and '35' in scenario S.3 - IT amenities grow and scenario S.19 - Decrease in Air Quality.

Several implications can be identified from the reweighting and scenario-informed scores for one single policy. For example, policy P.9 - Plan Multimodally appeared to be more favorable to the decision maker in the scenarios of S.2 - Sprawl Accelerates and S.17 Retirement. For scenario S.18 - Natural Disaster, S.3 - IT amenities grow and S.19 - Decrease in Air Quality the policy did not change in favourability significantly. Similar interpretation is informative for planners to evaluate the robustness of policies. And it could be enlightening for stakeholder groups that advocate different scenarios, and thus allow the stakeholder to understand how a policy impact may change with other scenarios that are influential to a region.

Figure 4.5-1 is a visualization of the results, showing the range of scores for each policy across all scenarios evaluated. The diamond represents the policy score in the baseline scenario. The sensitivity of the policy scores under different scenarios is represented by the vertical bars extending from the diamonds. The bars can also be viewed as the maximum and minimum scores 
of each policy under the influences of several scenarios. A taller bar suggests a relatively greater sensitivity of the policy impact to the scenarios. Many of the bars in figure overlap when compared, which indicates that significance of the upside and downside relative to the baseline score. This occurrence is mentioned by Montibeller et al. (2006) as the "inter-scenario robustness". The robustness of the policies varies in different scenarios, thus giving planners insights about the potential risks associated with policies.

The figure and table warrant the following observations. First, no scenario always produced the highest or lowest policy scores. It indicates that the scenarios do not equivalently affect all policies. The variety of impacts is expected because scenarios affect stakeholder and planners' perception of the performance of policies in different ways. Second, the length of bars representing the range of policy scores varied for each policy. This indicates a difference in the magnitude of scenarios' impact. Third, policy scores overlap between each other for a policy across the five scenarios. This indicates that different scenarios may produce similar impact differences on policies, and thus can be grouped together in future stages of planning.

Figure 4.5-2 provides the rank ordering of policies across the five scenarios. The median rankings (respect to all the six scenarios) are represented by the diamonds and the "error bars" across the diamonds represent the sensitivities of the policies rankings. It can be observed that some policies have a tall upside sensitivity bar relative to their median ranking, while others have a tall downside sensitivity bar to the median ranking. The upside and downside of the policies bar can be viewed as a characterization of risks inherent to the policy. Policies that associate with a high baseline ranking and a considerable volatility of rankings across scenarios may be of concern as sources of potential risk in transportation planning. Policies with a low baseline 
ranking and high rankings on other scenarios may be viewed as sources of potential positive surprise.

A sample of insights from the scenario-informed include: the top three policies based on the median ranking are P.13 - Invest in technology, P.15 - Plan Multimodally, and P.11 - Think Multimodally. The overall top ranked policies, P.13 - Invest in Technology and P.15 - Plan Multimodally, both consistently ranked at $1^{\text {st }}$ and $2^{\text {nd }}$ places throughout the scenarios. One regional analysis methodology was applied elsewhere to the scenario-based strategic analysis on other regions (Schroeder, 2009).

Additionally, the impact of the scenarios on the priorities of the policies is noteworthy. The knowledge of the magnitude of scenario effects on policy impacts can be used in several ways by planners and stakeholders, including planning iterative scenario-based analysis and allocating pre-caution resources. For this case study, scenario S.2 - Sprawl Accelerates has the largest impact on the overall policy rankings. Planners and stakeholders may at least consider paying more attention to this scenario in subsequent investigation, communication and decisionmaking. 
Table 4.5-6 Overall policy impact scoring of the statewide transportation policies in the case study 4.5.

Scenarios

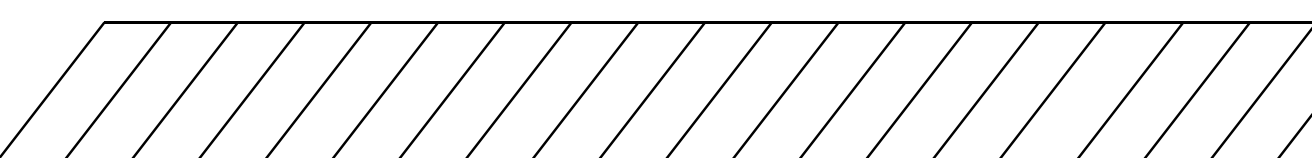
Default Criteria Weights

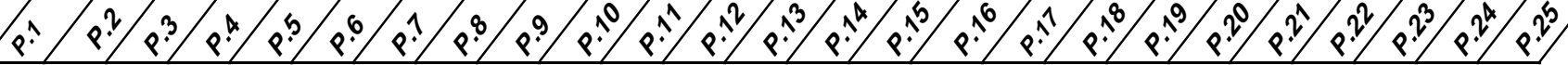

S.2 Sprawl accelerates

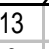

S.17 Retirement

S.18 Natural disaster

\begin{tabular}{l|l|l|l|l|l|l|l}
6 & 46 & 41 & 24 & 17 & 25 \\
6 & 46 & 42 & 23 & 18 & 25 & 0
\end{tabular}

\begin{tabular}{l|l|l|l|l|l}
34 & 31 & 21 & 35 \\
53 & 28 & 37 & 43 \\
53 & 28 & 38 & 42
\end{tabular}

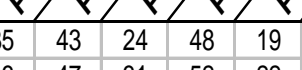

S.3 IT amenities grow

\begin{tabular}{|l|l|l|l|l|l|l|l|l|l|}
6 & 46 & 42 & 23 & 18 & 25 & 53 & 28 & 38 & 42 \\
\hline 6 & 46 & 20 & 42 & 17 & 45 & 25 & 39 & 28 & 36 \\
\hline
\end{tabular}

amenities grow

\begin{tabular}{|l|l|l|l|l|l|l|l|l|l|}
\hline 13 & 41 & 23 & 22 & 22 & 40 & 34 & 31 & 21 & 35 \\
\hline
\end{tabular}

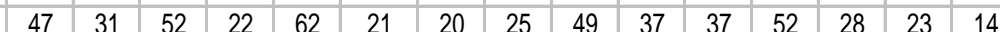

S.19 Decrease in air quality 


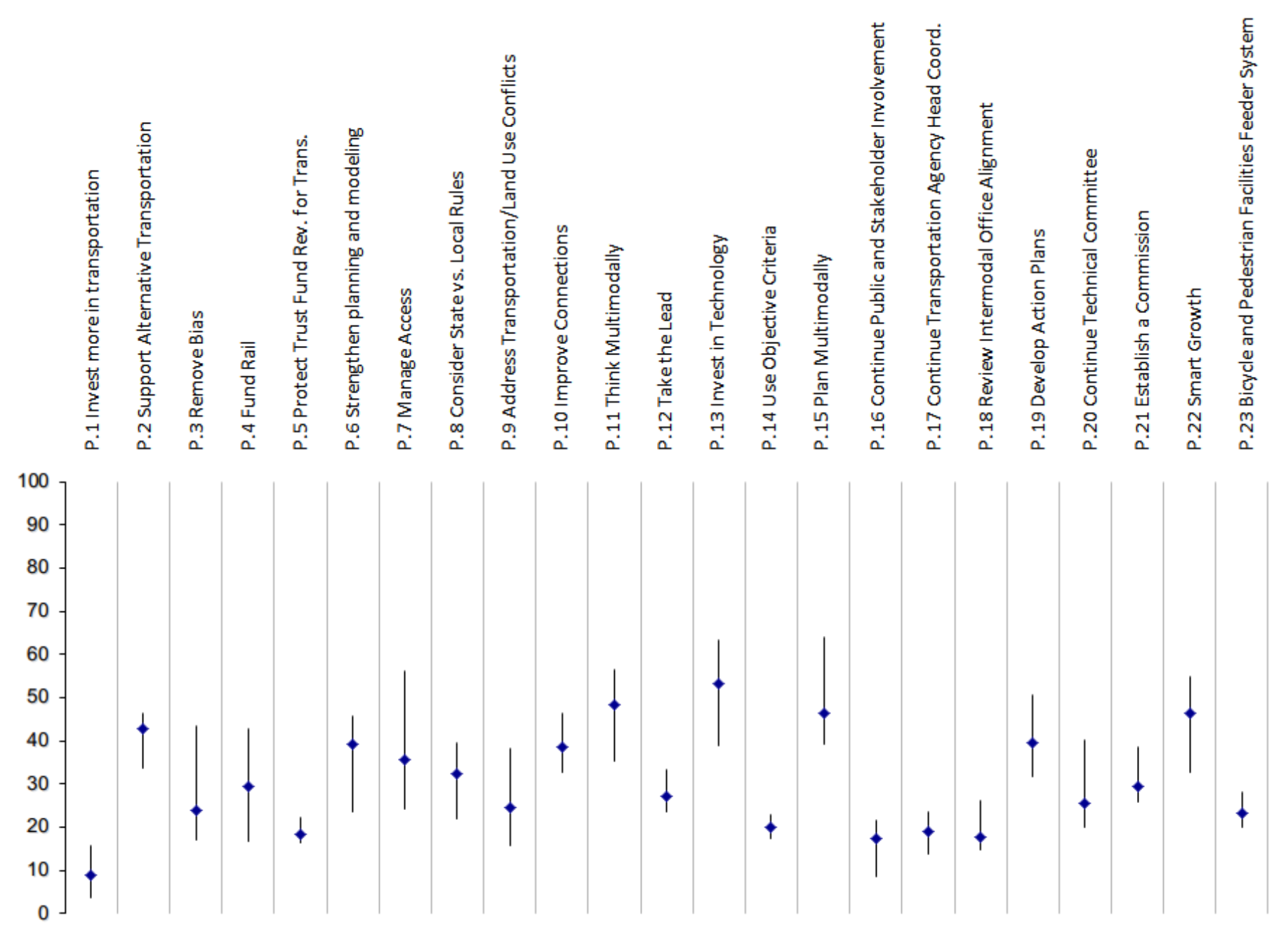

Figure 4.5-1 Range of policy impact scores with as-planned scenario policy score represented by diamonds in the case study 4.5 


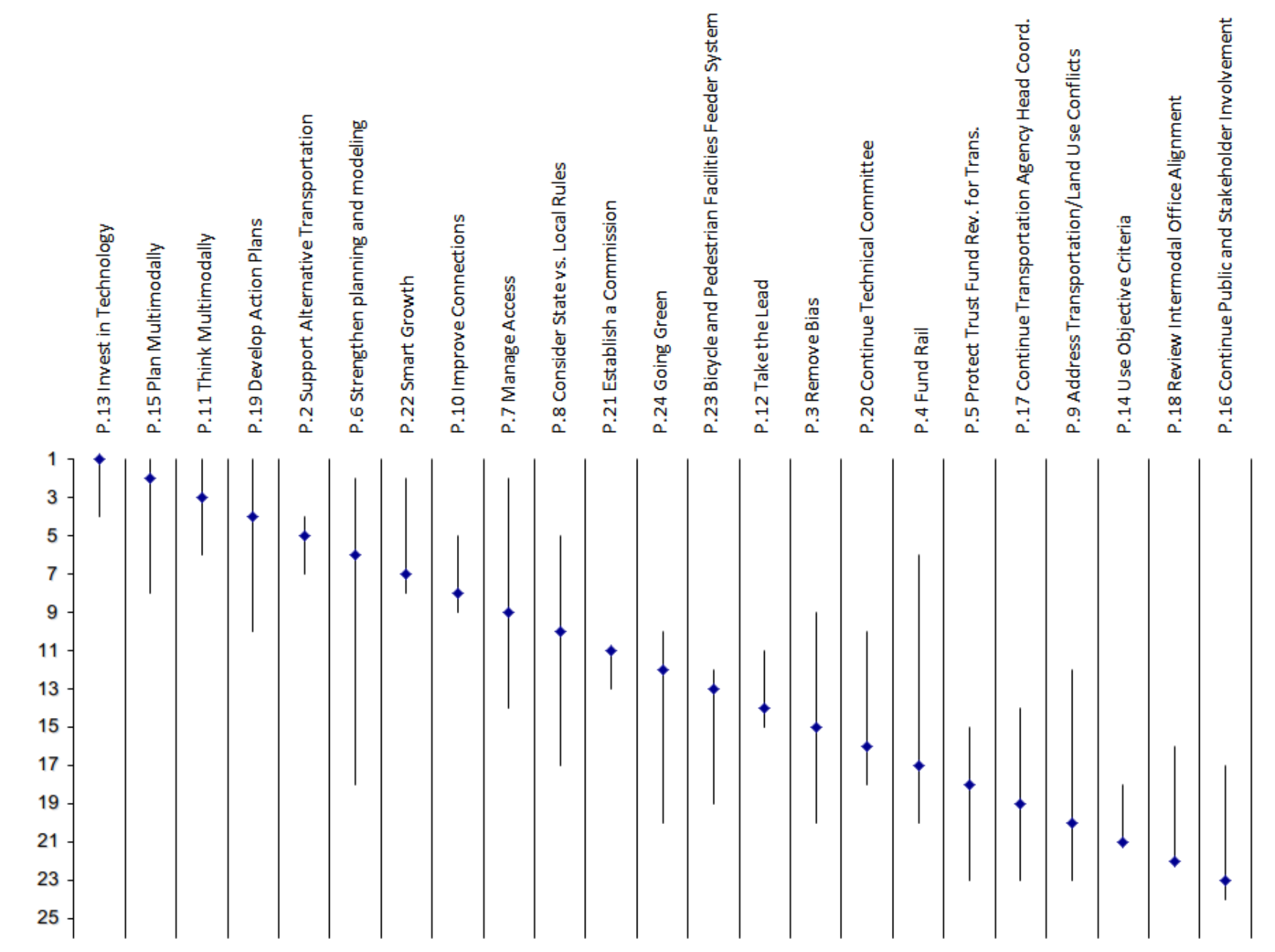

Figure 4.5-2 Rankings of policies with sensitivities to five scenarios represented by the height of the bars extending from the diamonds in the case study 4.5 


\subsection{Case Study: Vulnerability Assessment for Traffic Analysis Zones}

\subsubsection{Overview}

This case study is featured by the integration of Geographic Information System (GIS) into the analytical framework to minimize the dependency on expert opinions and the bias caused by improper usage of elicitation techniques.

The methodology presented here uses GIS to assess the vulnerability of different traffic analysis zone to several hazards. GIS is capable of offering efficient visualization and processing on data distributed spatially in a region, which may otherwise be time-consuming. Most of the required public and commercial data for climate change and social-economic changes related scenarios already exist in standardized format. Some of the data for individual regions or infrastructures may need to be collected on field. This case study will utilize these GIS based data to generate hazard maps give the boundaries of the areas that are mostly affected by different scenarios. These maps will be overlain to create a map showing the overall vulnerability.

The inputs for the variables in the analytical frameworks are obtained from a series of GIS based analysis and a two-hour meeting. The attendees of the meeting include demographic transportation analysis experts from VCTIR, transportation regional planners from HRTPO, and faculty members and research assistants of University of Virginia. 


\subsubsection{Criteria definition}

In order to evaluate the overall vulnerability to the emergent climate and social-economic changes of a particular traffic analysis zone, we have worked with the regional planning organizations and identified the criteria below. All criteria equally weighted for the purpose of demonstration

\section{Magnitude of sea level rise}

The impacts of sea-level rise are already evident in many coastal regions around the world, which includes coastal erosion, frequent inundation, increase of salinity in surface water, loss of coastal wetland and last but the not least various levels of socioeconomic impacts where transportation is a critical issue.

To assess the vulnerability of the region to sea level rise, the traffic analysis zones were classified into three classes based on the elevation and the sea-level rise projections:

- High risk: the TAZs with lowest $20 \%$ elevation;

- Low risk: the TAZs with highest $20 \%$ elevation;

- Medium risk: the TAZs with elevation between.

\section{Magnitude of storm surge}

Storm surge is a long wave motion produced by meteorological force that results higher water surface elevation relative to the elevation that is produced by normal astronomical tides. Hurricane or other tropical storms are one of main cause of this type of abnormal sea level rise. Storm surge is measured by subtracting the sea level in normal weather condition from the 
observed sea level during the event of the hurricane. Storm surge is reported to be the cause of 90\% deaths of all hurricane related fatalities (Shultz et al, 2005).

Vulnerability of TAZs to storm surge was evaluated based on the possibility to be inundated by hurricanes with different Saffir-Simpson Hurricane Wind Scale below:

- High risk: the TAZs that will be inundated by storm surge produced by a Category 1 hurricane (sustained winds speed: 74 to 95 miles per hour);

- Medium risk: the TAZs that will be inundated by storm surge produced by a Category 2 hurricane (sustained winds speed: 95 to 110 miles per hour);

- Low risk: the TAZs that will be inundated by storm surge produced by Category 3 hurricanes or above (sustained winds speed: greater than 110 miles per hour).

\section{Interstate highway and evacuation routes}

The interstate system plays an integral role in the road transportation connecting the whole nation thus providing mobility and ensuring efficiency in travel for not only general person movement, freight movement and but also plays significant role in national security. Besides these regular operations, interstate system is a crucial part of the hurricane evacuation route in the coastal region. Mid-Atlantic coastal regions that frequently experience hurricane each year, for example Hampton Roads have designated routes that are used for evacuation purpose in emergency situations.

Since these facilities are extremely important for the functioning of regional transportation system, their location can be utilized as criteria for assessing vulnerability of TAZs in the region: 
- High risk: the TAZs that have both interstate and hurricane evacuation route either crossing or passing their boundary;

- Medium risk: the TAZs with only evacuation route either crossing or adjacent to their boundary are ranked;

- Low risk: the TAZs with neither interstate or evacuation route crossing or adjacent to the boundary.

\section{Multimodal transportation facility}

An efficient multimodal transportation system is one of the key factors of success for the regional economy. Benefits of a well-functioning multimodal transportation system is experienced through reduction of overall transportation cost either by 1) reduced travel time or 2) by increased reliability of on-time shipment (Virginia Statewide Multimodal Freight Study, Final Report, 2010). In Hampton roads industries like construction, manufacturing and agriculture have big influence in the regional economic structure and these industries that heavily rely on freight movement for their success. Not only these industries are major regional employer but also have played key role in job creation. Relationship with multimodal freight transportation with these is strong and intertwined. Because of its geographic location this region has become increasingly important not only for the regional economy but also for the national economy; since it has number of important multimodal facilities like ports, cargo airports and also freight rail terminals.

Assuming that location of key multimodal transportation facilities play key role in vulnerability assessment of the region, rules were formulated to classify TAZs. 
- High risk: the TAZs with shortest $20 \%$ distance from a major regional multimodal transportation facility;

- Low risk: the TAZs with longest $20 \%$ distance from a major regional multimodal transportation facility;

- Medium risk: the TAZs with distance from a major regional multimodal transportation facility between.

\section{Population density}

Population was selected as one of the criteria for assessing vulnerabilities since it has a profound impact in the transportation facilities and also adds weight to the overall vulnerability of the region. Both sea level rise and storm surge due to hurricane pose greatest threat to the population of the coastal region, especially for the vulnerable group. Uncertainties regarding future climate change effects coupled with projected coastal population growth suggest incorporating this scenario based vulnerability assessment in planning programs (Schroeder and Lambert, 2010).

Though Hampton Roads area experienced a moderate growth when compared with national average for the 2000-2007 period; however it is expected to experience a higher growth in the coastal area in the next 23 years (Cambridge Systematics, 2010).

In order to assess the vulnerability index of the selected TAZs the population data set was categorized into three groups based on population density.

- High risk: the TAZs with more than 7501 people per square mile; 
- Medium risk: the TAZs with population density between 1001-7500 people per square mile.

- Low risk: the TAZs with population not more than 1000 people per square mile;

\subsubsection{Traffic analysis zones selection}

There are over twenty thousands of TAZs are defined in the coastal region of the Hampton Roads. For the purpose of demonstration, we pick a list of 50 TAZs to show how the methodology works in this case study. Without loss of generality, the 50 TAZs are intentionally evenly spread in terms of their geometric center. The list and the map of the selected TAZs are showed by Table 4.6-1 and Figure 4.6-1, respectively. 
Table 4.6-1 A sample of the 50 selected Traffic Analysis Zones in the case study 4.6

\begin{tabular}{|c|c|}
\hline $\begin{array}{c}\text { Traffic Analysis Zones (TAZs) } \\
\text { ID }\end{array}$ & County \\
\hline 1346 & James City \\
\hline 1326 & James City \\
\hline 1315 & James City \\
\hline 1311 & James City \\
\hline HJC001 & James City \\
\hline 100005 & Surry \\
\hline 1205 & Newport News \\
\hline 1198 & Newport News \\
\hline 1232 & Poquoson \\
\hline 1228 & Poquoson \\
\hline$\ldots \ldots$ & $\ldots \ldots$ \\
\hline 290 & Virginia Beach \\
\hline 129 & Norfolk \\
\hline 210 & Virginia Beach \\
\hline 497 & Portsmouth \\
\hline 510 & Sufflok \\
\hline 847 & Sufflok \\
\hline 147 & Norfolk \\
\hline 503 & Portsmouth \\
\hline 404 & Chesapeake \\
\hline 576 & Sufflok \\
\hline
\end{tabular}




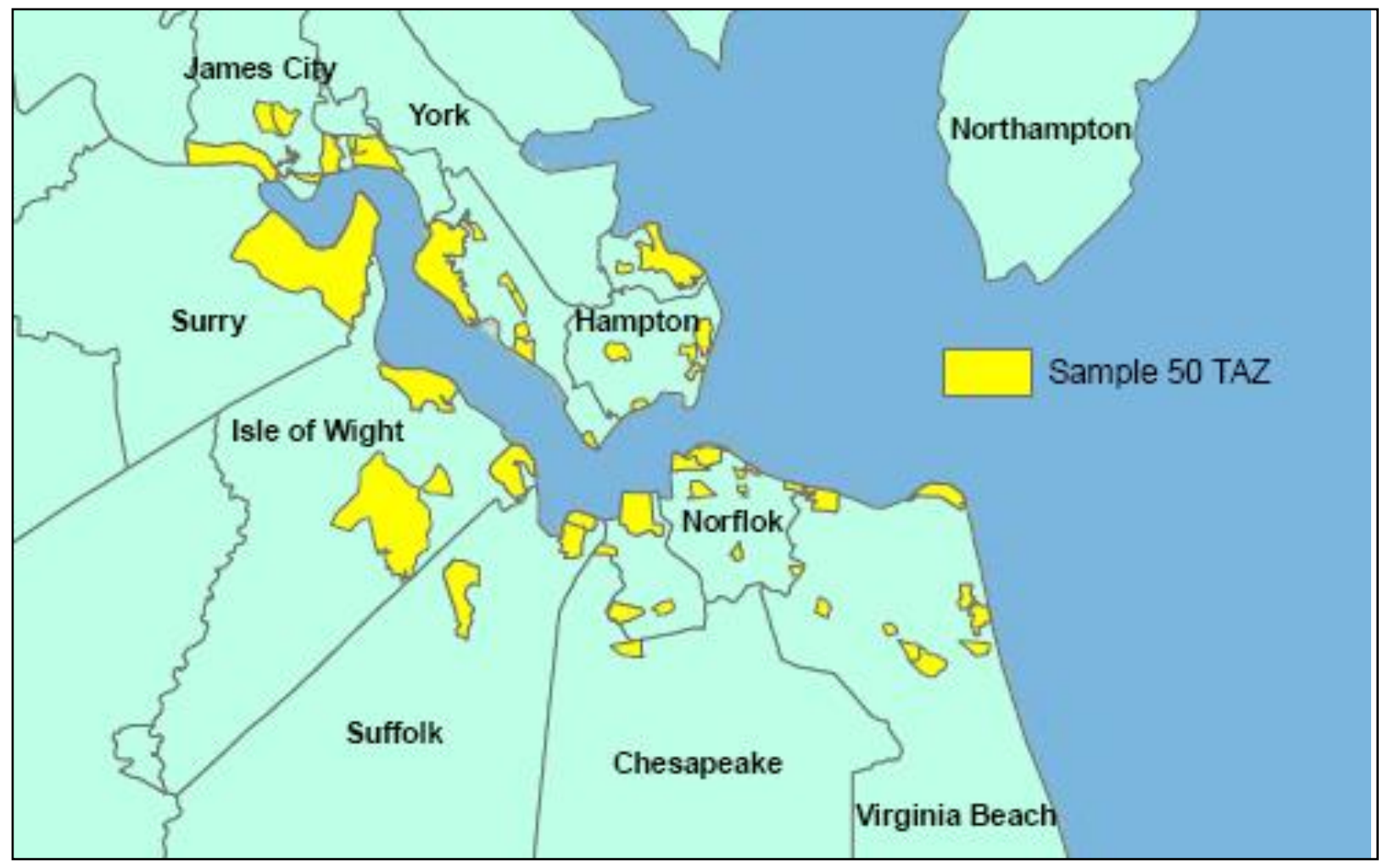

Figure 4.6-1 The overview map for the sampled 50 TAZs in the case study 4.6 


\subsubsection{Baseline assessment}

Different from the previous case studies, the baseline assessment result for the TAZ analysis is fully calculated according to the data provided in map layers from different data source.

The topographical data includes the Virginia elevation data titled "dem_va_shw_cm" were obtained from the US Environmental Protection Agency, published on February 2008. The elevation data is in raster data class and is relative to the spring high water of 2000 . The purpose of this data was to assess the vulnerability of coastal regions in Virginia to sea level rise. The highway map layer was obtained from the Virginia Department of Transportation. The data file includes information about local and state highways and highways with limited access that is freeways mainly interstates. The data file also includes the hurricane evacuation routes for the Virginia in the event of a hurricane and storm surge hits the coastal region. The hurricane evacuation route was developed by Virginia DOT with the help of Virginia Department of Emergency Management to minimize traffic congestion and ensuring safe evacuation of people at distressed area during the event of a hurricane. The Storm surge data was exported from the SLOSH database. As mentioned earlier the results from thousands of simulated storms are combined to produce MOMs that represents composite of the maximum storm surge heights for all simulated hurricanes of a given category. For the case study the MOMs for each category at high tide were selected from the SLOSH database and then exported as GIS file for the scenario analysis in ArcGIS. The population data was obtained from the online database of US Census Bureau. This dataset contained the latest population data from the US census 2010. Though information about specific number of people living in each census block was available in the dataset, population density was used in GIS to generate more logical output. Multimodal 
transportation asset map layer is obtained from the assets management system of Virginia DOT. The transportation asset data included ports, airports, railroad crossings, bridges. It is necessary to mention that for analysis all the assets of Virginia were not considered, since the scenarios considered have geographical limitations in terms having impacts on the assets.

The vulnerability assessment of the sample TAZ layer was performed for each of the five evaluation criteria developed for this case study. The risk maps for each of the criteria were produced using a color coding scheme of three colors, red representing high risk, yellow representing medium risk and green representing low risk. For example, Figure 4.6-2 shows the risk assessment results based on the sea level rise. In this figure the TAZs that are coded with red are assessed to be highly vulnerable to sea level rise since a sea level rise of $30 \mathrm{~cm}$ or less will inundate completely or part of this zone. The medium vulnerable zone are those that will be inundate with sea level rise of more than $30 \mathrm{~cm}$ but less than $60 \mathrm{~cm}$, in the Figure $4.6-2$ these zones are represented with yellow color. The rest of the TAZs in the sample layer are considered to have low vulnerability to sea level rise and coded with green color. Similar mapping scheme are followed to represent the vulnerability assessment results using all the other four evaluation criteria. Figure 4.6-3 represents vulnerability of traffic analysis zones to storm surge under different hurricane intensity. Figure 4.6-4 represents vulnerability assessment of the sample zone under rule developed for Interstate highway and evacuation routes dimension. Figure 4.6-5 shows vulnerable zones assessed by rule for multimodal transportation facility criteria. Vulnerability of analysis zones based on population density is demonstrated in the Figure 4.6-6. Finally the Figure 4.6-7 represents aggregated vulnerability of the traffic analysis zones combining the assessment of the five evaluation criteria. 
The result from the GIS-based multicriteria vulnerability assessment for the 40 traffic analysis zones under each criterion is shown in a tabulated format in Table 4.6-2 The GIS based baseline assessment for the 50 selected TAZs in the case study 4.6. 


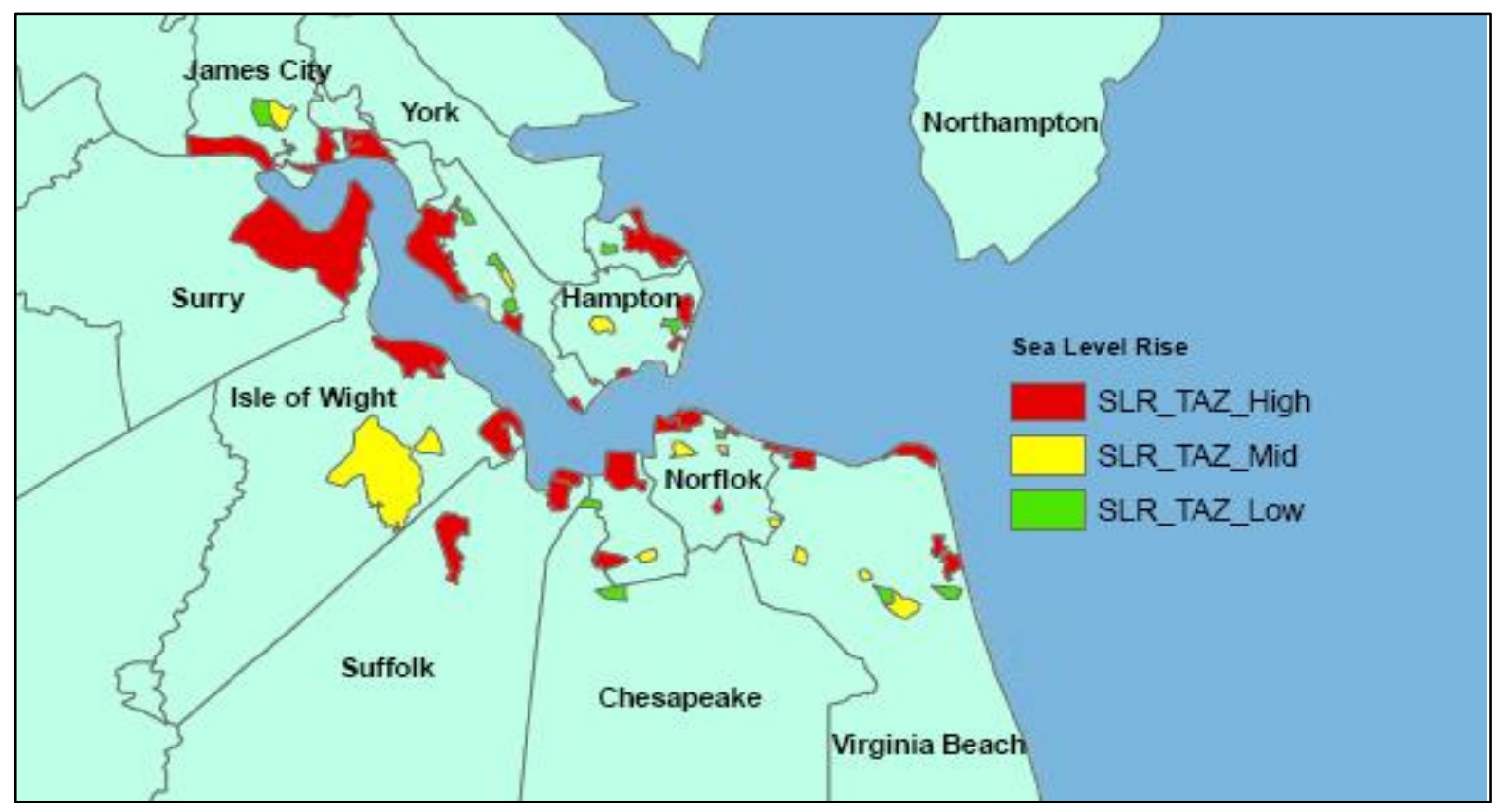

Figure 4.6-2: Vulnerability map based on sea level rise in the case study 4.6

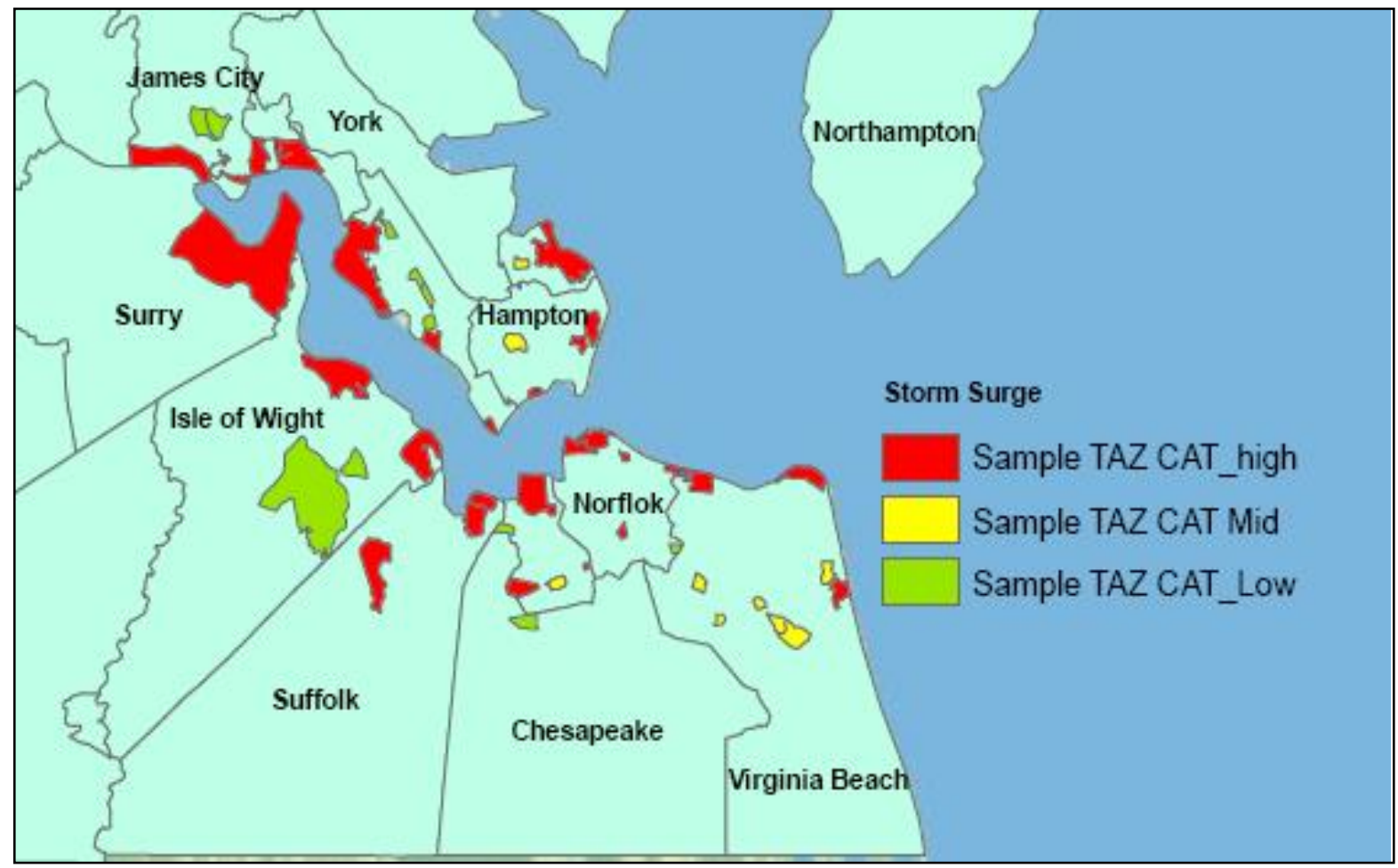

Figure 4.6-3: Vulnerability map based on hurricane intensity in the case study 4.6 


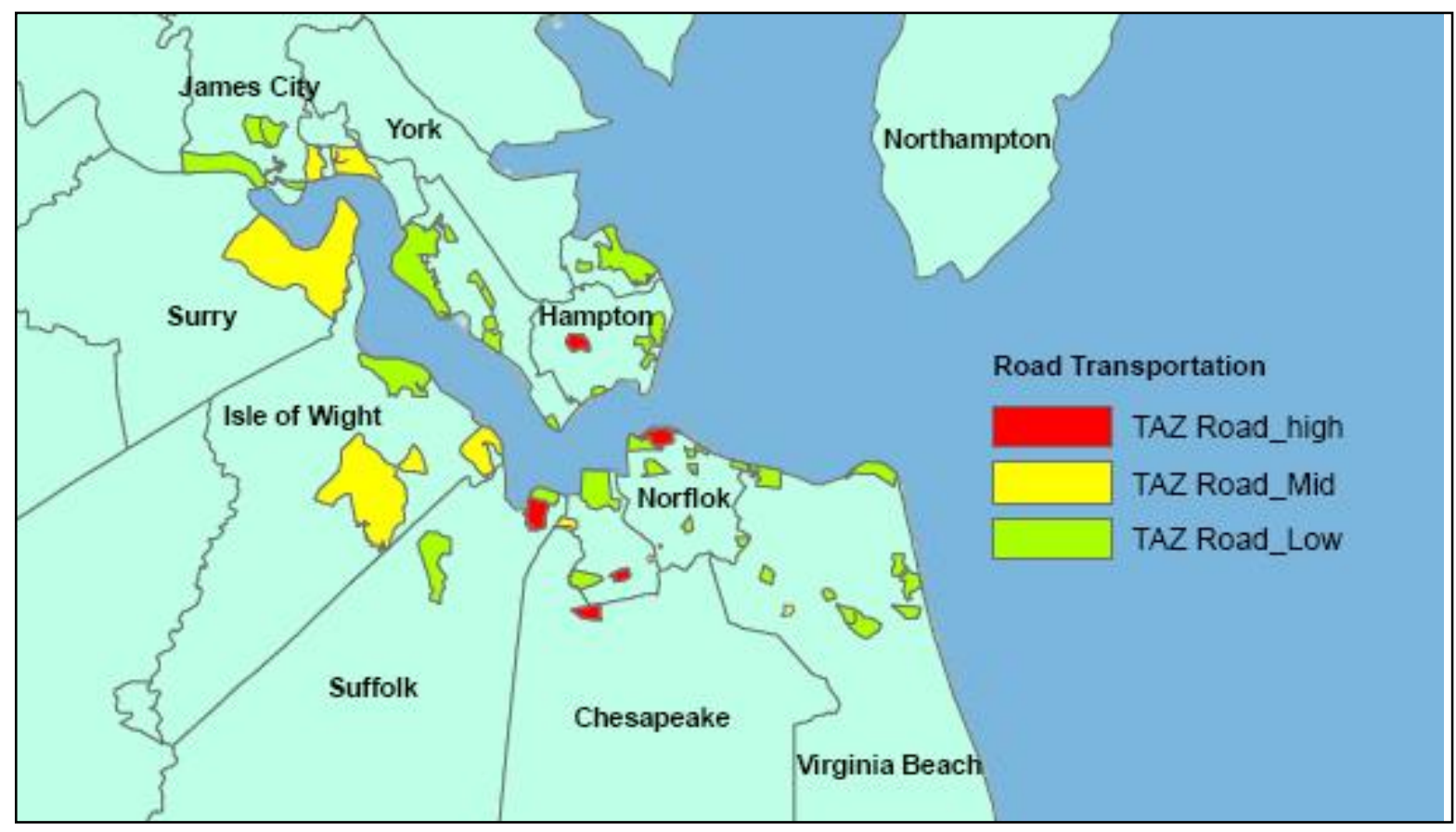

Figure 4.6-4: Vulnerability map based on road transportation in the case study 4.6

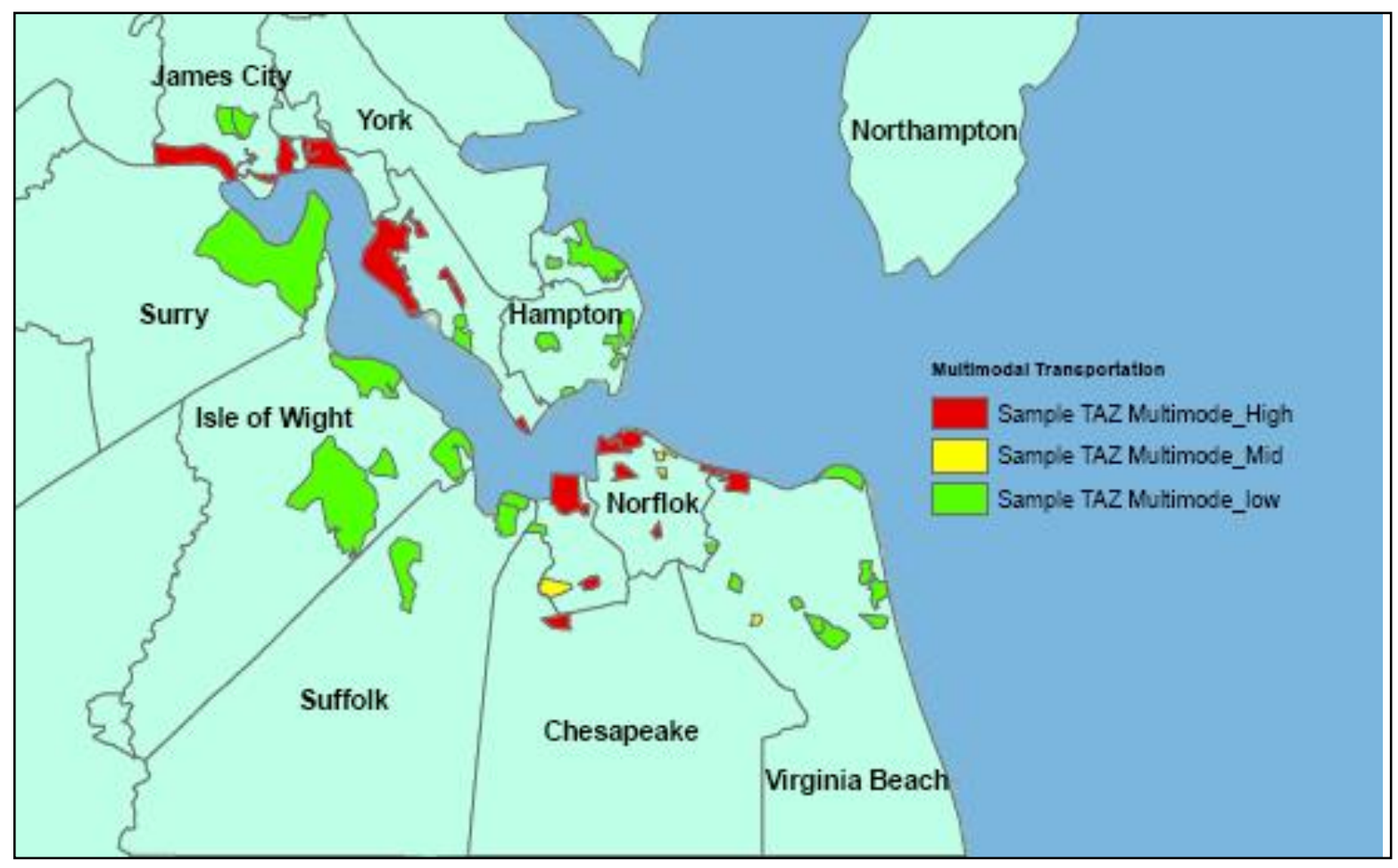

Figure 4.6-5: Vulnerability map based on multimodal facility in the case study 4.6 


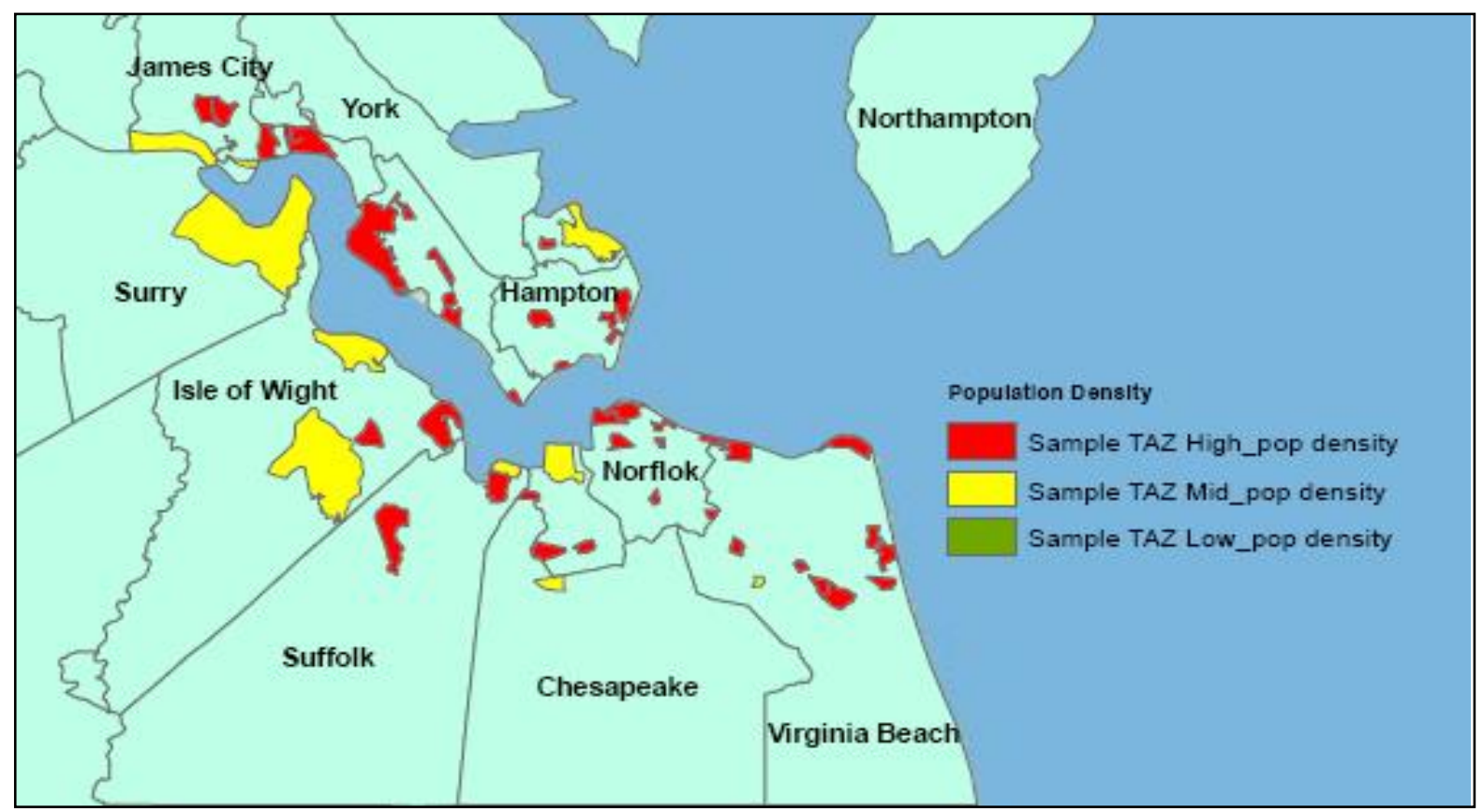

Figure 4.6-6: Vulnerability map based on population density in the case study 4.6

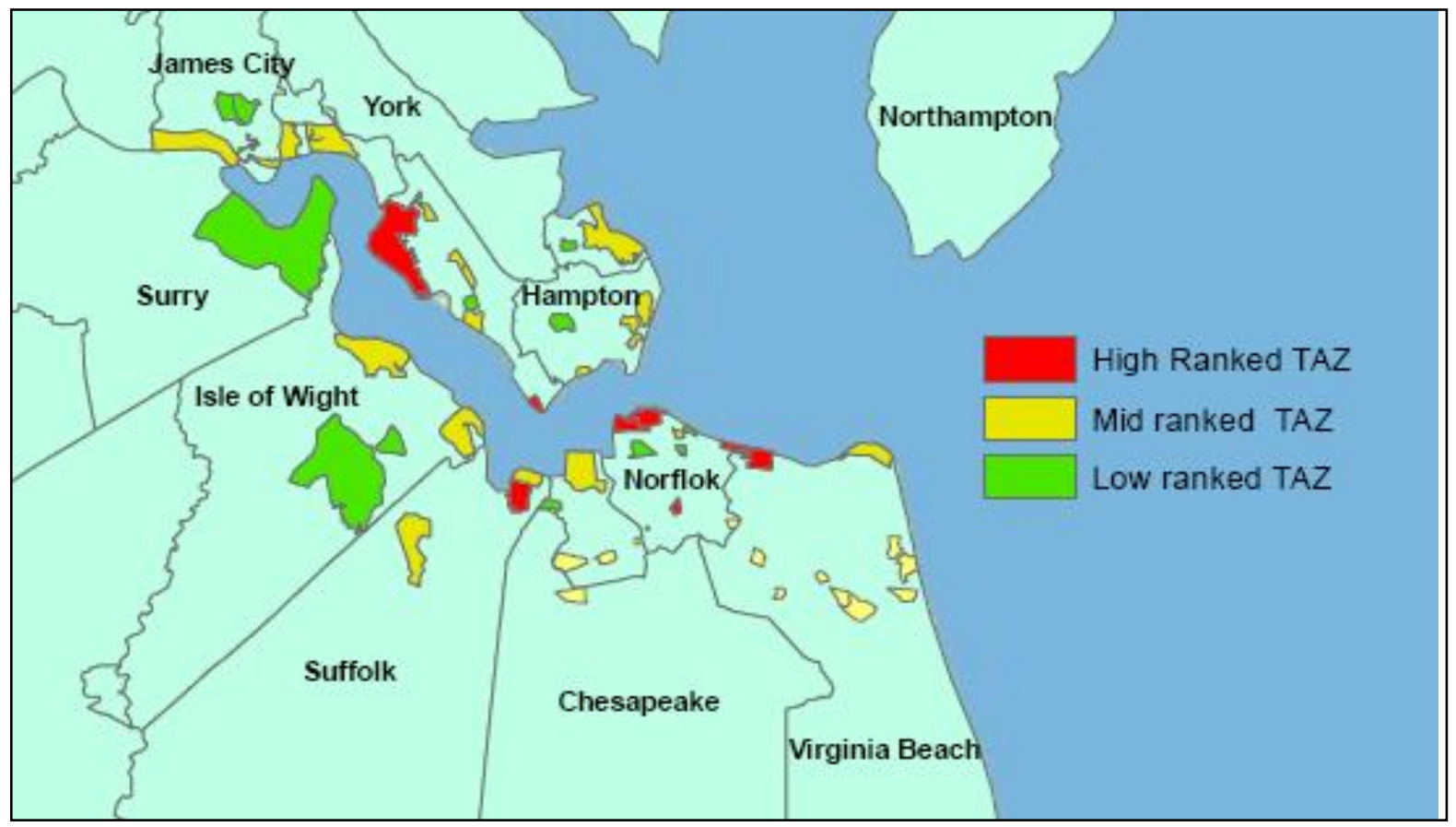

Figure 4.6-7: Aggregated vulnerability map based on all criteria in the case study 4.6 
Table 4.6-2 The GIS based baseline assessment for the 50 selected TAZs in the case study 4.6.

\begin{tabular}{|c|c|c|c|c|c|c|c|c|c|c|c|c|c|c|c|}
\hline \multirow{2}{*}{ Criteria } & \multicolumn{15}{|c|}{ Traffic Analysis Zone (TAZ) } \\
\hline & 1346 & 1326 & 1315 & 1311 & HJC001 & 100005 & 1205 & $\ldots$ & 497 & 510 & 847 & 147 & 503 & 404 & 576 \\
\hline $\begin{array}{l}\text { C1. Sea level is achieved } \\
\text { by considering this TAZ. }\end{array}$ & Low & Mid & High & High & High & High & Low & $\ldots$ & High & High & High & High & Low & Mid & High \\
\hline $\begin{array}{l}\text { C2. Storm Surge is } \\
\text { achieved by considering } \\
\text { this TAZ. }\end{array}$ & Low & Low & High & High & High & High & Low & $\ldots$ & High & High & High & High & Low & Low & High \\
\hline $\begin{array}{l}\text { C3. Road Transportation } \\
\text { is achieved by } \\
\text { considering this TAZ. }\end{array}$ & Low & Low & Mid & Mid & Low & Mid & Low & $\ldots$ & Low & Low & High & Low & Mid & Low & Low \\
\hline $\begin{array}{l}\text { C4. Multimodal is } \\
\text { achieved by considering } \\
\text { this TAZ. }\end{array}$ & Low & Low & High & High & High & Low & High & $\ldots$ & High & Low & Low & High & Low & Low & Low \\
\hline $\begin{array}{l}\text { C5. Population Density } \\
\text { is achieved by } \\
\text { considering this TAZ. }\end{array}$ & High & High & High & High & Mid & Mid & High & $\ldots$ & Mid & Mid & High & High & High & High & High \\
\hline
\end{tabular}




\subsubsection{Scenario analysis and results}

Table 4.6-3 describes the emergent conditions and the assembled scenarios for this case study. An extensive review of technical reports and transportation planning reports produced by HRTPO and HRPDC helped to list the conditions. Sea level rise and storm surge were identified as the top concerned climatic issue for the region in this demonstration. Non-climatic issues like economic recession, increased wear and tear in public infrastructure and increased traffic demand were considered in constructing the five scenarios to have a more realistic scenariobased planning perspective for the planning organizations.

Table 4.6-4 provides the shift of preference in criteria that corresponds to each of the five scenarios based on comparison with the baseline scenario. This adjustment of criteria preference is achieved through a collaborative input from professionals who are involved in the regional transportation planning, risk analysis of Climate Change scenarios and environmental engineering. For example Scenario 5 has a minor increase of preference on c3. Interstate highway and evacuation routes, and minor increase for c4. Multimodal facilities. These inputs are translated into multipliers to new weights for each scenario with a similar methodology with the previous case studies. Based on the newly generated weights, the additive values for the TAZs are calculated and the TAZs are ranked accordingly across the five defined scenarios. Figure 4.6-8 and Figure 4.6-9 are the graphical representation of the scenario-informed analysis results. The diamonds represent the scores / rankings in the baseline scenario. The sensitivity of the scores / rankings under different scenarios are represented by the vertical bars extending from the diamonds. 
Some TAZs are ranked high for all scenarios, such as TAZ No. 1198, No. 1121, No. HNR002, No. 99, and No. 147. But for some of the TAZs, the rankings are very sensitive to different scenarios. For example, TAZ No. 1018 ranked 28th under the baseline scenario and even as low as 36th for the sea-level rise alone scenario (Scenario 1). However under the scenario combining climate change and increased traffic demand (Scenario 5), it is ranked $3^{\text {rd }}$ and become one of the most critical geographic region. This high sensitivity may worth further investigating for planners and obtain more understanding about characteristics of a region. Another example of similar significant ranking changes is TAZ No. 1315, which has baseline ranking 9 th, but ranked at $22^{\text {nd }}$ for the scenario combining climate change and increased traffic demand.

Apart from only considering the priority in terms of vulnerability of TAZs, it can be helpful to identify scenarios that were most influential to the priorities. Table $4.6-5$ provides the disruptive scores for the five scenarios considered in this demonstration. The values represent the sum of square ranking changes of the ranking values compared to the baseline ranking. The higher the score the more influential it is in rearranging the vulnerability rankings of the TAZs. Scenario 5 which combines climate change and increased traffic demand is most disruptive. 
Table 4.6-3 Definition of conditions and scenarios constructed in the case study 4.6.

Conditions Scenarios

\begin{tabular}{|c|c|c|c|c|c|}
\hline Increase in sea level rise & $\mathrm{X}$ & $\mathrm{X}$ & $\mathrm{X}$ & $\mathrm{X}$ & $\bar{X}$ \\
\hline Increase in storm surge & $\mathrm{X}$ & $\mathrm{X}$ & $\mathrm{X}$ & $\mathrm{X}$ & $\mathrm{X}$ \\
\hline \multicolumn{6}{|l|}{ Increase in precipitation } \\
\hline \multicolumn{6}{|l|}{ Increase in storm water } \\
\hline \multicolumn{6}{|l|}{ Increase in storm frequency } \\
\hline \multicolumn{6}{|l|}{ Increase in days below freezing } \\
\hline \multicolumn{6}{|l|}{ Increase in extreme heat days } \\
\hline \multicolumn{6}{|l|}{ Increased occurrence of drought } \\
\hline \multicolumn{6}{|l|}{ Economic recession } \\
\hline No further increase on federal government debt cap & & $\mathrm{X}$ & & & \\
\hline Increased wear and tear on public infrastructure & & $\mathrm{X}$ & & & \\
\hline \multicolumn{6}{|l|}{ New technology for maintenance / inspection } \\
\hline \multicolumn{6}{|l|}{ Increase in traffic demand } \\
\hline \multicolumn{6}{|l|}{ Increase in area tourism } \\
\hline \multicolumn{6}{|l|}{ Population growth } \\
\hline Energy shortage & & & & $\mathrm{X}$ & \\
\hline Changes in land use regulation & $\mathrm{X}$ & & & & $\mathrm{X}$ \\
\hline Increased infectious disease occurrence & & $\mathrm{X}$ & $\mathrm{X}$ & & \\
\hline Increased loss of forest and plant life & & & $\mathrm{X}$ & & \\
\hline Increased mortality of native animal species & & & & & $\mathrm{X}$ \\
\hline
\end{tabular}

* A " $\mathrm{X}$ " indicates that the condition in that row is included in the scenario listed in the column. 
Table 4.6-4 Criteria relative importance adjustment for new scenarios in the case study 4.6

\begin{tabular}{|c|c|c|c|c|c|}
\hline \multirow{2}{*}{ Criteria } & \multicolumn{5}{|c|}{ Scenarios } \\
\hline & s1 & s2 & s3 & s4 & s5 \\
\hline C1. Sea level & minor increase & & & & \\
\hline C2. Storm Surge & minor increase & & & & \\
\hline C3. Highway and Evacuation & & & MAJOR INCREASE & & MAJOR INCREASE \\
\hline C4. Multimodal & & & & MAJOR INCREASE & minor increase \\
\hline C5. Population Density & & minor increase & & & \\
\hline
\end{tabular}

Table 4.6-5 The disruptiveness of scenarios for the priority of TAZs in the case study 4.6

\begin{tabular}{lc}
\hline Scenarios & \multicolumn{1}{c}{$\begin{array}{c}\text { Value } \\
s_{1} . \text { Scenario 1 }\end{array}$} \\
$s_{2}$. Scenario 2 & 423 \\
$s_{3}$. Scenario 3 & 1663 \\
$s_{4}$. Scenario 4 & 624 \\
$s_{5}$. Scenario 5 & 1201 \\
\hline
\end{tabular}




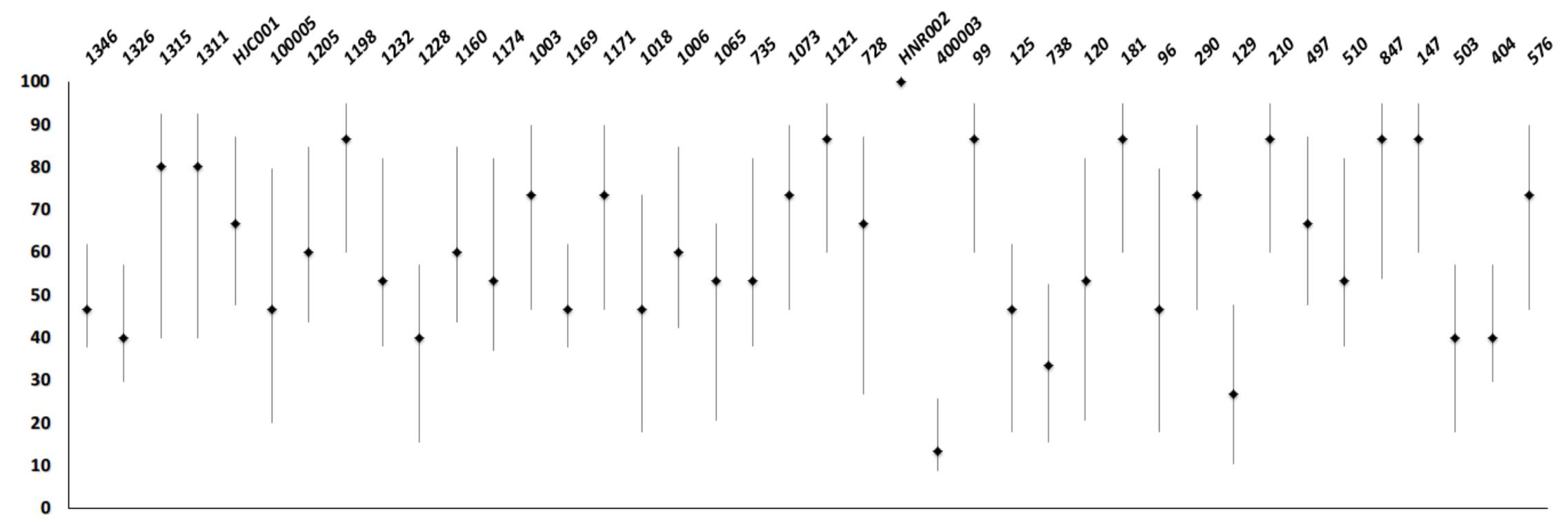

Figure 4.6-8 The overall risk scores of all 50 selected TAZs across the future scenarios in the case study 4.6 


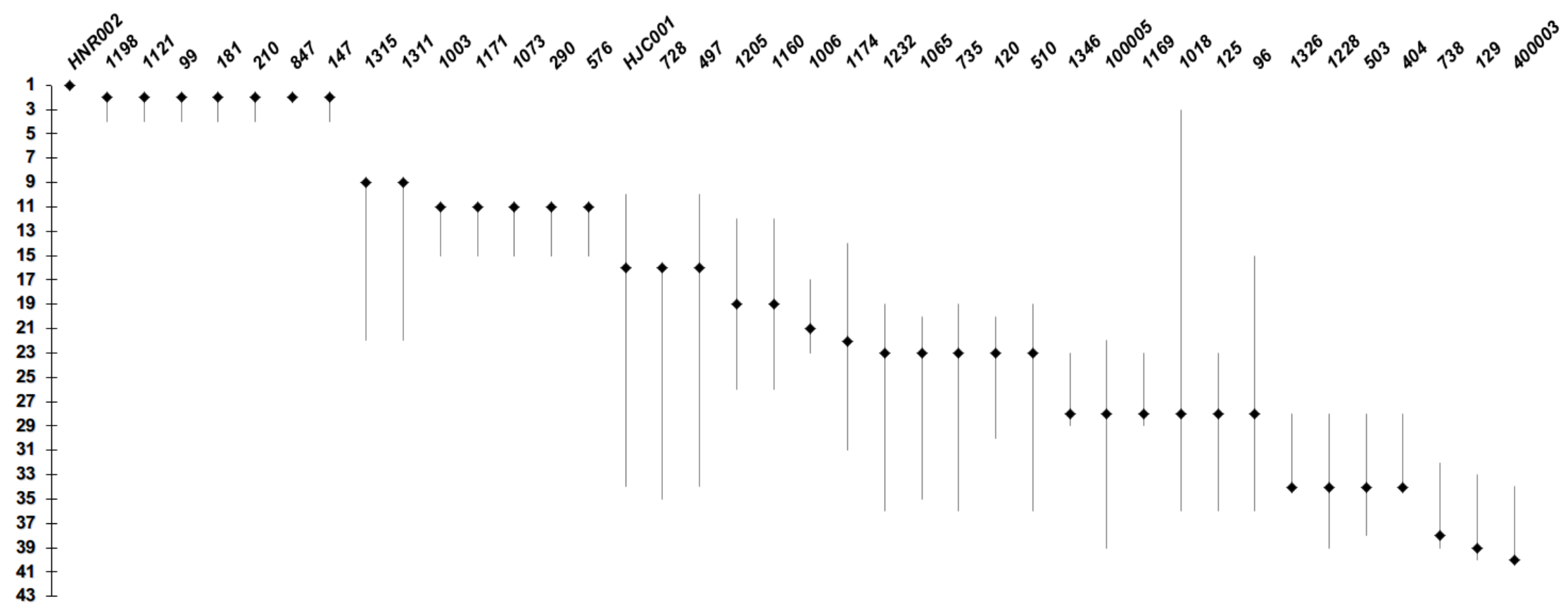

Figure 4.6-9 The overall ranking of all 50 selected TAZs across the future scenarios in the case study 4.6 


\subsection{Integrated Analysis across Multiple Case Studies}

The case studies above quantified the influence of scenarios using different methods. In this section, we will perform integrated analysis on the transportation system of the Hampton Roads via the scenario based priority-setting and vulnerability assessment.

By replicating calculation based on the scenario influence defined based on Kendall tau-b, we can obtain the system vulnerability profiles from all four perspectives: infrastructure management, project selection, multimodal policy making, and demographic analysis. Table 4.8-1 describes the metric values and overall rankings of the influence of scenarios $s_{1}$ through $s_{5}$ from all four perspectives. Figure 4.8-1 provides a visual method to help interpret these results by radar charts.

The results indicate that the analysis from four perspectives agrees on that the Hampton Roads transportation system is vulnerable to the scenario $s 2$-Climate change combining with economic recession. This conclusion calls for further scientific investigation or simulation studies to reveal more detailed information for the construction of early warning mechanism, redundancy and some other adaptation decisions. The scenario $s_{1}-$ Climate change only is considered the least significant from all perspectives. This conclusion emphasizes that the planning agency should be aware of spending too much resources to only study the climate change related threats, because relatively, the damage enlarged by the amplifying interaction between climate change and other conditions is more risky.

On the other hand, the results from different perspectives failed to reach consensus about the impacts of scenario $s_{3}, s_{4}$ and $s_{5}$. The disagreements reveal the essential advantage of multiperspective assessment. If the HRTPO only perform vulnerability assessment from the 
perspective of project selection, the further investigation and policy-making will be built on the biased results, which overstate the importance of preparing for scenario $s_{5}$ - Climate change combined with increased traffic demand. Because the analysis angle is unilateral, the vulnerability assessment from project selection perspective will also not be able to discover the facts such as the system assets are remarkably vulnerable to scenario $s_{3}-$ Climate change combined with accelerated wear and tear; and the multimodal policy portfolio can be devalued once scenario $s_{4}-$ Climate change combined with ecosystem stress manifests itself in the future.

\subsection{Lessons Learned from Case Studies}

As the real-world applications of the introduced approach, the case studies described in the Section 4.3 to Section 4.7 confirmed the significance of the research and also provided important "lessons learned" for the implementation of this approach. Table 4.8-2 describes a summary of these lessons learned. 
Table 4.8-1 The $\phi$ values and rankings of the influence associated to five scenarios from four perspectives.

\begin{tabular}{|c|c|c|c|c|c|c|c|c|}
\hline \multirow{2}{*}{ Scenarios } & \multicolumn{2}{|l|}{ Project } & \multicolumn{2}{|l|}{ Assets } & \multicolumn{2}{|l|}{ Policies } & \multicolumn{2}{|l|}{ TAZs } \\
\hline & $\phi$ Value & Ranking & $\phi$ Value & Ranking & $\phi$ Value & Ranking & $\phi$ Value & Ranking \\
\hline$s_{5 .}\left[s_{1}+\right.$ Traffic demand $]$ & 0.276 & 1 & 0.242 & 3 & 0.199 & 9 & 0.186 & 11 \\
\hline$s_{2} .\left[s_{1}+\right.$ Economic recession $]$ & 0.228 & 4 & 0.216 & 6 & 0.226 & 5 & 0.201 & 8 \\
\hline$s_{3} .\left[s_{1}+\right.$ Wear and tear $]$ & 0.133 & 14 & 0.273 & 2 & 0.157 & 12 & 0.121 & 15 \\
\hline$s_{4 .}\left[s_{1}+\right.$ Ecosystem stress $]$ & 0.114 & 18 & 0.118 & 17 & 0.212 & 7 & 0.194 & 10 \\
\hline$s_{l}$. Climate change only & 0.102 & 20 & 0.136 & 13 & 0.119 & 16 & 0.105 & 19 \\
\hline
\end{tabular}

Project - the perspective of future project selection

Assets - the perspective of infrastructures and assets management

Policies - the perspective of multimodal policy analysis

TAZs - the perspective of traffic analysis zone (TAZ) supported demographic issues analysis 

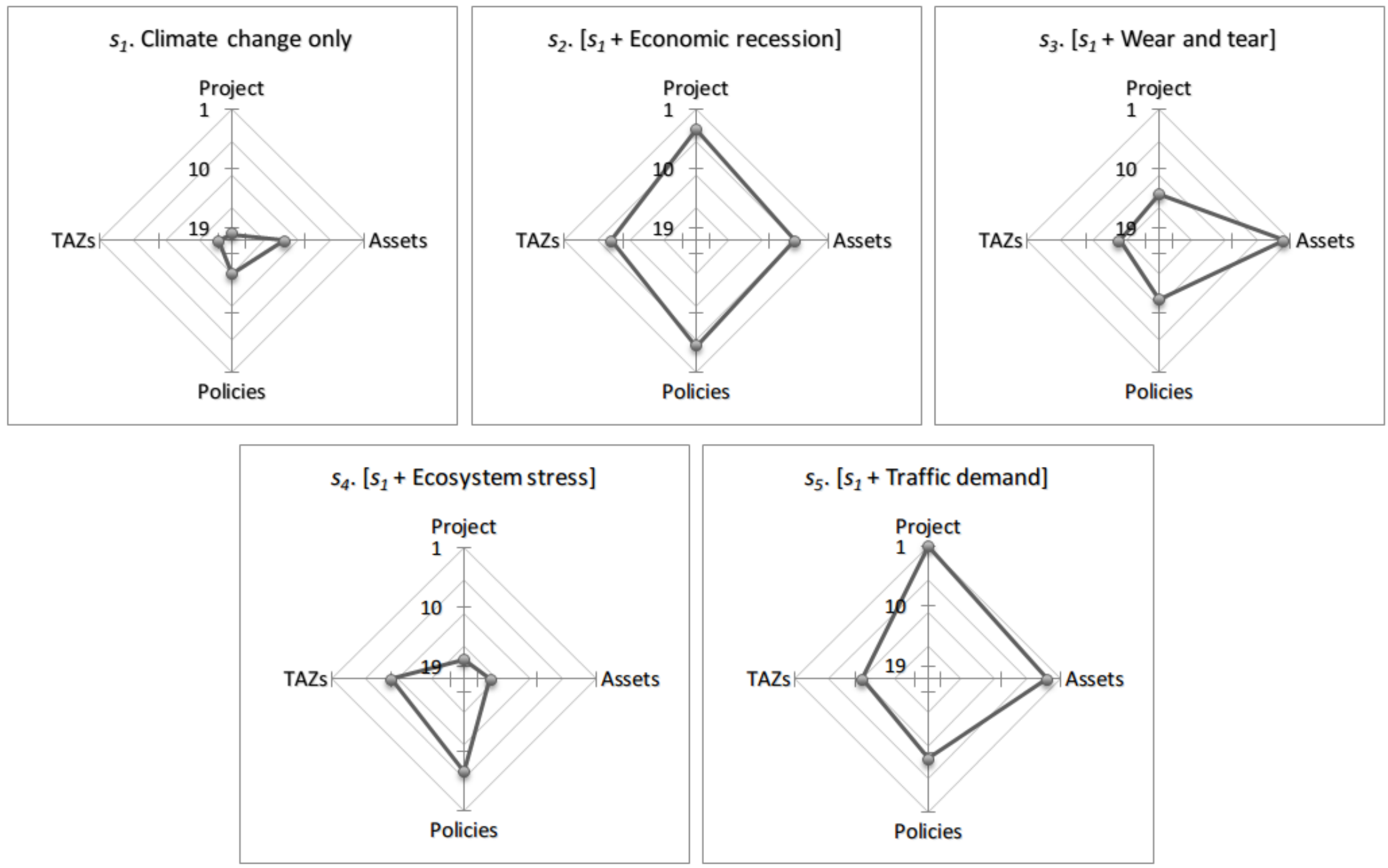

Figure 4.8-1 Demonstration of integrated framework with the influences of five scenarios to priority setting across four system perspectives: Projects, Assets, Policies, and Geographic Zones (TAZs). 
Lessons learned

\begin{tabular}{|c|c|}
\hline Identify existing works & $\begin{array}{l}\text { At the initiation of the projects, significant scientific work was found } \\
\text { including sea level rise and storm surge, by identifying vulnerable } \\
\text { transportation infrastructure, species, ecosystems, regional economic } \\
\text { sectors, and socioeconomic structure of the Hampton Roads region. } \\
\text { These research results and documents were used as an important } \\
\text { source of the inputs of the decision analysis framework. }\end{array}$ \\
\hline $\begin{array}{c}\text { Incorporate the analysis } \\
\text { with existing planning } \\
\text { process }\end{array}$ & $\begin{array}{l}\text { Scenario analysis has been introduced to planning agencies for } \\
\text { several years. However, scenario based studies had not yet been } \\
\text { incorporated to the process of updating the regional Long Range } \\
\text { Transportation Plan, Transportation Improvement Plan and other } \\
\text { plans for which the maintenance is mandatorily required. } \\
\text { We recognized the Long Range Transportation plan as an appropriate } \\
\text { venue for addressing impacts of climate change in the regional } \\
\text { transportation system. The analysis can be integrated into different } \\
\text { existing planning efforts with different planning horizon (Figure } \\
\text { 4.8-2). }\end{array}$ \\
\hline $\begin{array}{l}\text { Elicit inputs from } \\
\text { experts and staff }\end{array}$ & $\begin{array}{l}\text { We recognized inputs from experts and planners are a proper } \\
\text { balancing point between the demanding need for existing scientific } \\
\text { and engineering resources and the limited modeling, investigative } \\
\text { and human resources assigned to address deep uncertainties. }\end{array}$ \\
\hline $\begin{array}{c}\text { Involve multiple } \\
\text { perspectives on system } \\
\text { analysis }\end{array}$ & $\begin{array}{l}\text { The integrated vulnerability assessment was proven to be more } \\
\text { informative than analysis from a single dimension to decision- } \\
\text { making. And the involvement of stakeholders with different } \\
\text { perspectives expanded the source of inputs to decision models. The } \\
\text { workshops and meetings facilitated the establishment of relationships } \\
\text { between agencies. }\end{array}$ \\
\hline
\end{tabular}




\section{Lessons learned}

\section{Description}

We found that climate change influences the priorities of

Consider the combining

effect of different

driving forces transportation planning more significantly through combining with other factors including economics, ecology, transportation demand, condition of transportation infrastructure, land regulations, energy usage, market place and other factors. The factors combine as scenarios that influence priority settings from the several dimensions.

Education and training of officials and staff of the planning agencies (in the case studies including regional Metropolitan Planning The importance training Organization, the Hampton Roads Planning District Commission and and education etc.) are considered important to the successful implementation. A website was built to publish the user-friendly, editable Microsoft Excel workbooks and relevant documents. 


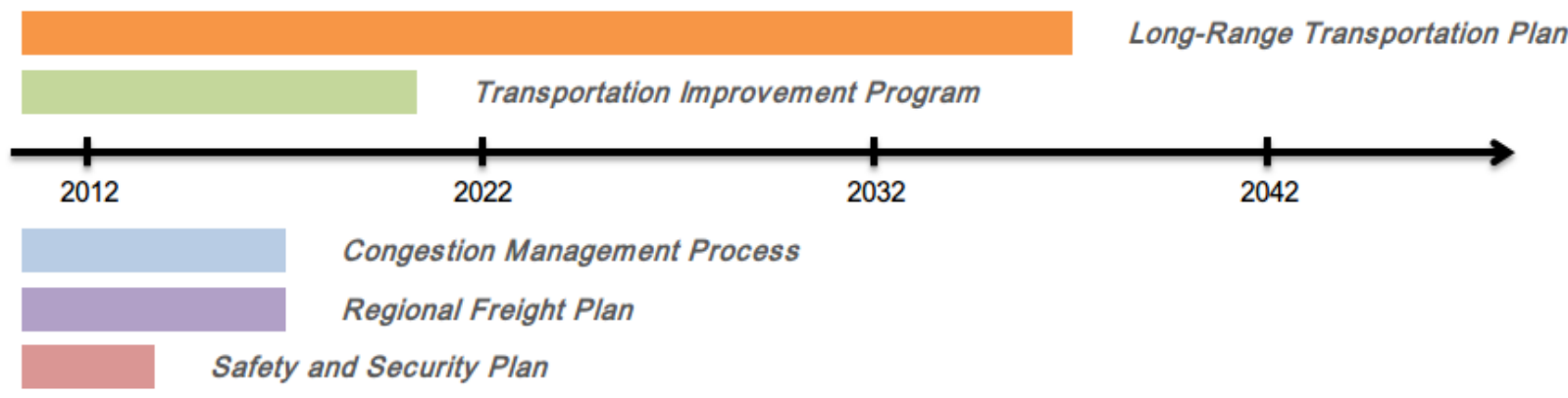

Figure 4.8-2 Time horizons of different plans maintained by regional transportation planning agencies 


\subsection{Chapter Summary}

This chapter demonstrated the analytical framework introduced in Chapter 3. Section 4.2 briefly introduced the background of the four cases studies covered in this chapter. Section 4.3 described an application on infrastructure vulnerability assessment of a coastal region. Section 4.4 described an integration of the methodology with the existing project selection process of long range transportation planning of the Hampton Roads Planning Organization (HRTPO). Section 4.5 described how this methodology was applied to address deep uncertainties for a state-wide multimodal policy analysis project. Section 4.6 demonstrated the integration of geographic information system (GIS) and the approach on identifying vulnerable traffic analysis

zones (TAZ). Section 4.7 described the integrated vulnerability assessment based on the inputs of the four case studies above, which is able to provide insights on system vulnerability from multiple perspectives. 


\section{Chapter 5. Discussion}

\subsection{Chapter Overview}

This chapter will assemble and analyze various commentaries on the analytical framework and case studies. The commentaries were collected from peer reviews of the published and submitted papers and with advice of the PhD advisory committee. Section 5.2 will discuss how we can validate the decisions made in the case studies and introduced methodology. Section 5.3 will discuss the applications of the analytical framework in other real-world cases. 


\subsection{Validation of the Introduced Analytical Framework}

Validation of decision support frameworks has been a controversial topic. The fundamental debate is on whether a decision should be evaluated by the outcome, or according to the process by which the outcome has been obtained (George and White, 1992).

Most practitioners tend to evaluate a decision by the direct outcome. And according to George and White (1992), good decision outcomes add the credits of the corresponding decision making process. However, evaluating a decision method by the outcomes may face some questions, for which structured and well-defined answers can be nontrivial to find in real-world cases. An incomplete list of these questions include: 1) what is an acceptable outcome; 2) how an outcome can be better or worse; 3) whether all possible outcomes are explicitly definable; 4) whether all the outcomes are measurable. Moreover, the inferences conclusion can be draw from outcome to the decision process may be problematic. Because a good outcome may occur due to chance factors and thus does not necessarily imply that the corresponding decision processes were statistically reliable and beneficial; correspondingly, an unfavorable outcome may not necessarily be evidence for an improper decision process. The issues above do not mean to be exhaustive, but they are sufficient to indicate the potential dangers in judging decisions solely by the outcomes.

Decision analysis researchers suggest that a decision should be evaluate based on the process by which it was reached. For instance, Simon (1978) argued that "economics has largely been preoccupied with the results of rational choice rather than the process of choice". Similar views were expressed by Majone (1977), Vlck et al. (1984), and Wright and Murphy (1984). In

past two decades, there has been a steady growth in research on the processes of making judgment and choice. "Process effectiveness" has been used as a metric to assess the quality of a 151 
variety of decision processes. (Rohrbaugh, 2005; McCartt and Rohrbaugh, 1995; Einhorn, 1980; Einhorn and Hogarth, 1981; Kahneman, et al., 1982; Payne, 1976; Svenson, 1979).

The debate is still on-going. No studies in this area have provided convincing evidence or reasoning to determine whether decisions should be evaluated by outcome or process. Currently, a widely accepted opinion is that the both perspectives are relevant, while their relative importance may vary depending on the specific decision problem. For long-term transportation planning (30-year planning horizon), it is more effective and efficient to reply on the evaluation of the decision-making process.

Bell et. al (1988) suggested two criteria for evaluating a decision process. French and Rios Insua (French and Insua, 2000) extended the criteria to a five criteria system, including axiomatic basis, feasibility, robustness, transparency and compatibility with a wider philosophy. Table 5.2-1describes the assessment of the introduced analytical framework under the criteria. 
Criteria for decision process

\begin{tabular}{|c|c|}
\hline Axiomatic basis & $\begin{array}{l}\text { The axioms behind MAVT such as preference indifference, } \\
\text { existence of weak order preference relation, have been verified } \\
\text { in stakeholder meetings. }\end{array}$ \\
\hline Feasibility & $\begin{array}{l}\text { The elicitation techniques and the calculations are practical and } \\
\text { easy to implement. } \\
\text { A series of software workbook have been created to improve the } \\
\text { usability of the decision process. } \\
\text { The realities and limitations human behavior have been taken } \\
\text { into consideration during elicitation processes to reduce the } \\
\text { cognitive load and potential biases. }\end{array}$ \\
\hline Robustness & $\begin{array}{l}\text { Sensitivity analysis are performed on the value of weights } \\
\text { adjustment multiplier and the natural languages statements on } \\
\text { the criteria importance shifting, to make sure whether the results } \\
\text { are sensitive to the inputs. }\end{array}$ \\
\hline Transparency & $\begin{array}{l}\text { The document for the decision process and the supporting } \\
\text { software workbook are published to the stakeholders so that they } \\
\text { understand the analysis procedure and find it meaningful. }\end{array}$ \\
\hline $\begin{array}{l}\text { Compatibility with the } \\
\text { decision context }\end{array}$ & $\begin{array}{l}\text { The analytical framework helps decision makers to understand } \\
\text { the characteristics of individual decision alternatives, the } \\
\text { influence of a scenario, and the overall vulnerability of the } \\
\text { system to a group of future scenarios. The information is } \\
\text { compatible with the concerns of regional planners. Moreover, } \\
\text { the framework leaves space for the negotiation between } \\
\text { stakeholders. }\end{array}$ \\
\hline
\end{tabular}

\section{Introduced analytical framework}


While a number of literatures try to develop criteria to evaluate the effectiveness of a decision process, client feedback can be a direct and reliable indicator of a successful decision process. Among the feedback we collected for the case studies ${ }^{14}$, the regional planners and stakeholders highly appreciated the informativeness of multi-scenario and multi-perspective insights provided by the analytical framework to the transportation system and individual decision alternatives. The feedback from regional planners at HRTPO pointed out that the framework has improved the planning capability of the MPO, and helped the MPO to perform the roles. Moreover, the ease of using the framework and the modest amount of efforts required to obtain the information are also rated as the advantages of this analytical framework over existing methodologies (e.g. traditional unstructured scenario analysis) addressing deep uncertainties in strategic planning. Using structured survey to collect feedback is a part of future works and will be described in Section 6.4.

\subsection{Applications of the Analytical Framework}

\subsubsection{The "escape fish" of scenario analysis}

The scenarios are assembled by the emergent future conditions obtained via document analysis and stakeholder interviews. They represent the threats which are concerned by the stakeholders and planners. The quantified influence of scenarios will provide suggestions for further resource allocation and the establishment of pre-caution system.

${ }^{14}$ Most feedback from the participants of the applications are collected in unstructured and informal communications. 
However, any decision analysis models are essentially a simplification of a reality which includes significant insights from selected aspects (Keeney, 1992). In this sense, the scenarios and the process of scenario construction are merely tools to aid the decision making under uncertainties. Theoretically, it is possible that even with the help of the calibration and inspiration during the scenario construction process, the stakeholders and planners failed to identify one or two critical scenarios (e.g. the New York state before hit by the hurricane Sandy). This failure in the scenario identification usually results in losses in property and human lives.

The introduced analytical framework is designed to avoid this failure in three ways:

First, we encourage a high cardinality set of the set of emergent conditions. During the analysis, the analytical framework tries to incorporate emergent conditions from all possible sources, include historical data, documents, expert opinions, public involvement and others. These conditions will be categorized and sorted to be used as the ingredients of scenario construction.

Second, the scenario analysis is iterative with iterations through the steps of scenario construction and scenario filtering. At the very beginning of the scenario construction, large amount of explorative scenarios can be introduced, or even generated with the aids from computer system. In each iteration, the scenarios and critical emergent conditions will be revisited so that no scenarios are arbitrarily abandoned. The iterative scenario refining means that ordering the scenarios is able to support the iterative process which is proposing rough scenarios, ordering and filtering scenarios, refining the retained scenarios, and proposing more specific scenarios based on the retained scenarios. This iterative process will be helpful to facilitate organizational communication and learning. 
Third, the scenarios are suggested to "bound" the uncertainties. In most cases, the scenarios retained in iteration are most influential ones. This scenario refining rule is to make sure the retained scenarios bound the plausible risks. These scenarios will usually suggest relatively aggressive investments on relevant investigation and pre-caution projects. Thus even the future is not directly hit by any of the scenarios, the precaution and investments inspired during the scenario analysis may still be able to help the mitigation and adaptation to the threats.

\subsubsection{Issues related to technology transfer}

The analytical framework is developed and tested in the context of regional transportation planning; however, it is generally applicable to address scenarios deep uncertainties (e.g. climate change, economic recession and etc.) in multicriteria prioritization of assets and infrastructure management agencies. A series of software workbooks and relevant documents, technical reports, academic publications were developed for the case studies of the Hampton Roads, and are available via the internet to other agencies (Figure 5.3-1). The workbook enables redefining the decision alternatives, the criteria and the emergent conditions which can be used to assemble scenarios. The method of analysis in other applications would be expected to be similar to, and to benefit from, the case study presented in this dissertation. 


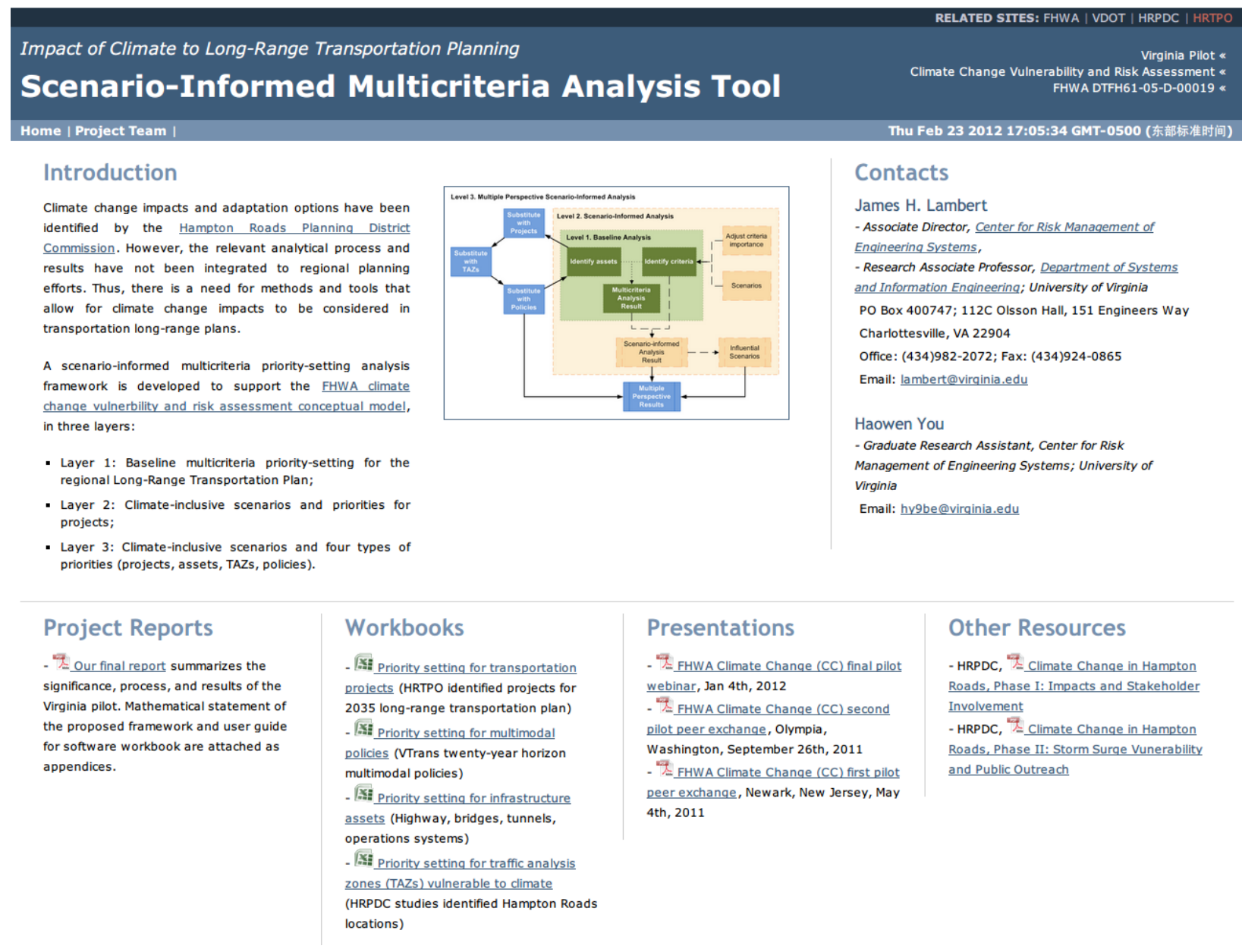

Figure 5.3-1 The website providing training and education materials for the implementation and transferring of the introduced framework 


\subsubsection{Natural disasters such as hurricane Katrina and Sandy}

In this section, we will discuss the improvements the introduced analytical framework is able to bring to us, when addressing natural disasters such as hurricane Katrina and Sandy.

Hurricane Katrina in 2005 was one of the most deadly hurricanes in the history of the United States. Over 1,800 people were killed; up to 600 thousands of families were homeless. The total damages and monetary loss exceed 248 billion dollars. Losses on economic, environmental and social aspects were unmeasurable yet believed to be huge. Hurricane Sandy in 2012 caused more than 132 deaths on the U.S. mainland. The damage was over 70 billions of dollars. Due to flooding and storm surge, Sandy affected 24 U.S. states. 8.5 million homes lost power. Amtrak canceled the service of Acela Express, Northeast Regional, and Keystone (Burton and Michael, 2005). Airlines canceled a total of 19,729 flights (Burton and Michael, 2005).

The both hurricanes underlined the fragility of the aging American infrastructure. And the limited investment in the prediction and preparation due to the recent rarity of hurricanes is believed to be among the most important reasons.

The analytical framework introduced in this dissertation first will help stakeholders to reach consensus on the potential risks. From the philosophy of this dissertation, the both disasters are the result of combination of climate and non-climate conditions. Although continuous sealevel rise and global temperature rise are observed, but the climate change may still be a controversial term for decision maker. Questions such as whether hurricanes like Katrina and Sandy are the results of the 30 billion tons of carbon dioxide do not have universally accepted answers yet. However, when this question mark combined with population and activity increase 
at coastal area, the statement that this region is exposed to significant risks should be consensus. Planners do not even have to believe in human activities-caused climate change, to see the need to plan for the hurricanes.

Second, with the scenario combining hurricanes and increase in coastal activities identified, the regional planners will be able to identify the densely populated zone, infrastructures of the power system that are vulnerable. Pre-caution system may be established and redundant resources can be prepared with the information. Moreover, critical evacuation routes will also be identified and thus draw more attention to the emergency action plan and continuity plan for hurricanes. And with more perspective involved in the analysis, we could identify the initiatives to educate coastal residences and agencies like hospitals prepare redundant resources. Also the framework may help to develop better resilience to the coastal infrastructure systems, just as introduced by Lincoln Institute of Land Policy, the Consensus Building Institute and Bio-Era, RPA after the hurricane Sandy (RPA, 2013).

Third, the analytical framework will facilitate the inter-agency relationship building. According to the feedback to Federal Emergency Management Agency (FEMA) workshops after the hurricane Sandy, the most important outcome of the workshops was not any particular policy or procedure changes. It was the bringing together all stakeholders and relevant organizations (US FEMA, 2012). This creates a network of relationships which would boost the effectiveness and efficiency of actions for the next big disaster. 


\subsubsection{To facilitate the vulnerability assessment of large single infrastructures}

In this section, we will discuss how an organization in charge of a large single infrastructure such as a dam and a power station (e.g. the Fukushima Nuclear Power Plant, and the Chernobyl Nuclear Power plant), could benefit from the introduced analytical framework.

The best usage of this analytical framework is to analyze the vulnerability of a system consists of a group of loosely correlated infrastructures or other decision alternatives competing for resources. The framework will be able to provide multi-perspective vulnerability assessment to uncertainties. As by-product, the decision maker will obtain better understandings on individual alternatives and the external threats. Therefore for large infrastructures such as dams and power stations, this analytical framework may be most applicable to the vulnerability assessment conducted by planning or management organizations of the whole dam / power station sector on a region or national scale (e.g. Dams Sector Specific Agency).

For the vulnerability and risk assessment on single infrastructure, the analytical framework may also be applicable by decomposing the infrastructure into sub-systems. These sub-systems can be feed into the analytical framework as decision alternatives. With proper risk evaluation criteria and emergent conditions identified, this framework is expected to help the understanding of the threats on this infrastructure and thus support decision-making.

However, the decision analysis model in this framework was built on a few independent assumptions. For single infrastructure such as dam and power station, due to the strong correlation and cooperation between sub-systems, the method may lead to biased conclusions. Other traditional risk analysis tools such as event tree and fault tree analysis may serve the purpose better (Hartford and Baecher, 2004). 


\subsection{Chapter Summary}

This chapter provided discussions on the introduced analytical framework and case studies. Section 5.2 discussed how we can validate the decisions made in the case studies and introduced analytical framework. Section 5.3 discussed the applications of the analytical framework in other real-world cases. 


\section{Chapter 6. Summary and Conclusions}

\subsection{Chapter Overview}

This chapter will describe the conclusions of the research and offer directions for future work. The sections of this chapter are organized as follows: Section 6.2 will review the purpose and scope of the dissertation; Section 6.3 will detail the theoretical, methodological, and application contributions of this effort to systems engineering and risk management literature; Section 6.4 will describe opportunities for future study.

\subsection{Review of Purpose and Scope}

This dissertation has introduced an analytical framework to perform vulnerability assessment on transportation system and integrate the assessment results from multiple 
perspectives. This framework extends previous efforts (Karvetski et al., 2009; Karvetski et al., 2011a, b, c; Lambert et al., 2012a, c; Martinez et al., 2011; Schroeder and Lambert, 2011 You et al., 2012), and adopts methods including scenario analysis, multicriteria decision analysis and rank distance quantification. The framework provides an approach to help local/regional planning agencies handle the balance between limited investigative resources and the demand for comprehensive and integrated analysis.

We demonstrated the framework in the context of long-range transportation planning. In the demonstration, we identified the four perspectives to assess the system vulnerability, including assets management, project selection, policy analysis and demographic issues. We have then demonstrated the how the system vulnerability is profiled from a single perspective project selection. This single perspective analysis also revealed the robustness and sensitivity of candidate projects as a by-product. By replicating this analysis for the other three perspectives, we obtained the multi-perspective vulnerability profile of the system. The results suggest that when taking different perspectives, vulnerability assessment may identify different influential stressors. Adaptation resource allocation and policy-making can be more rational when they are informed by vulnerability assessment results from multiple perspectives.

When it comes to assessing system vulnerability to a threat as complex as climate changes, it is meaningless to argue that any analytical approach should become the dominant discourse. The framework can be limited in application by various characteristics, for example, if experts are untrusted, or if the region is in shortage of active stakeholders and public involvement. Nevertheless, we do see distinct advantages of this introduced framework, in terms of the modest resources required and comprehensive assessments provided. This framework is considerably applicable as a "dialectic" approach for local- or regional-scale vulnerability 
assessment. For future works, we will emphasize proving more implications on the directions about adaption policies, especially for short-term policies. We will refine the elicitation process to ensure that representative and useful local knowledge are extracted and utilized. From the technique side, we will extend current scenario influence metric to reflect the relative importance of priority changes taken place on top and bottom alternatives. In this case, the top alternative falling to the $10^{\text {th }}$ place and the 91 th alternative falling to the $100^{\text {th }}$ will not be considered equally important. Furthermore, we will also investigate the correlation of the scenario impacts from different perspectives.

\subsection{Research Contributions}

This dissertation summarizes theoretical, methodological and application contributions disseminated in the literature (Lambert et al, 2011, 2012; You et al. 2013a; 2013 b; Hayat et al., 2013). Table 6.3-1 describes these contributions of this dissertation. 
Contributions of this dissertation

Reviewed the state of the art of analysis of deep uncertainties. Pointed out that

the balance between limited resources and the objective of comprehensiveness is the key to the success of analysis addressing deep uncertainties.
Addressed in chapter

Chapter 1

Identified a need to involve several system perspectives when performing priority setting and vulnerability assessments.

Chapter 3

Introduced a Kendall-tau-distance methodology, to support the quantification of scenario influence on weak order priority-setting, and the inter-perspective Chapter 3 comparison of scenario influence.

Incorporated unstructured knowledge via document analysis and expert

opinion elicitation as the inputs for scenario identification and vulnerability

Chapter 4 assessment.

Chapter 5

Described how to filter, retain and refine scenarios via an iterative process, to facilitate transparent and reliable converge of scenarios.

Chapter 3

Integrated the assessment of deep uncertainties as a part of existing planning processes, so that the framework can be implemented with moderate extra efforts, comparing to other methodologies that make scenario analysis a Chapter 4 separate process.

Utilized geographic information systems as source of inputs and result interpretation tool of a scenario-informed decision analysis framework

Chapter 4

Applied and verified the analytical framework on a series of real-world case studies. Software workbooks and a group of lessons learned / best practice are Chapter 4 thus obtained to support the methodology transferring to other areas.

Chapter 5 


\subsection{Future Work}

This section will discuss several research opportunities exist based on the philosophy, methods, and results of this dissertation effort.

\subsubsection{Quantitative and computerized scenario identification}

As discussed in Section 5.3.1, scenario identification is a critical phase for analysis addressing deep uncertainties. The quality and coverage of the scenarios identified is crucial to the subsequent priority-setting and vulnerability assessment.

The scenario identification approach introduced in this dissertation incorporates inputs from unstructured sources including internal documents, existing scientific research and experts opinions. Additionally, the approach emphasizes the importance of iteration on the comprehensiveness of scenario set. However, the effectiveness of this approach (i.e. whether all influential and highly possible scenarios can be identified) highly depends on the availability of the documents, research results and quality of expert opinion elicitation. Models and experience are hardly possible to be shared across agencies and applications. Moreover, the current purely qualitative approach is very hard to be computerized and thus can be time-consuming.

Bryant and Lempert (2009) proposed an approach called "scenario discovery". The approach characterizes the set of scenarios which are described by easy-to-interpret combination of uncertain model input parameters. Statistical analysis and data mining algorithms are utilized to help this discovery process. Discovery scenarios can be added to a library and provide quick reference for other applications. Despite the approach still has "significant potential for improvement" (Bryant and Lempert, 2009), later literature proved quantitative and computerized 
methods can smooth the transfer of knowledge on scenario identification between different agencies and areas, and boost the efficiency of scenario identification processes.

\subsubsection{Survey-based analytical framework assessment}

As described in Section 5.2, direct feedback from the users of this analytical framework, i.e. the regional planners, researchers from planning agencies and other stakeholders, may be the most convincing information to evaluate the success of a decision process. Figure 6.4-1 describes a sample of the questions in such survey analysis. In future work, we can perform a formal survey process. The questionnaire for the survey can be either paper-based or electronic. And the questions in the survey may collect feedback such as:

- Did the stakeholders / experts find any strong benefits and issues?

- Did the stakeholders / experts feel the process and results are useful or problematic?

- Would the stakeholders / experts be willing to introduce the analytical framework to colleagues and peers? Would they use it in the future planning cycles?

- Were the stakeholders / experts able to understand the results? How useful are the results?

- Did the stakeholders / experts feel the elicitation process is too demanding on the completeness of information?

- Did the stakeholders / experts feel the framework is generally easy to use?

- Did the stakeholders / experts find the analytical framework is generally more useful / easier to use than other existing methodologies?

- Did the stakeholders / experts feel the analytical framework enhance the inter-department and inter-agencies communication? 
2. Extent of top-down versus bottom-up influence in your organization during problem-solving processes (a) MARA: How do you rate the MARA decision analysis in terms of bottom-up versus top-down influence? (" $\left.\mathrm{M}^{\prime \prime}\right)$

(b) Ideal: How should problems similar to the one approached by the MARA team be ideally solved in your organization in terms of bottom-up versus top-down influence? (" $\mathrm{I}$ ")

(c) Status Quo: With how much bottom-up/top-down influence would the decision problem at hand or similar problems have been solved with the existing processes/methods? ("SQ")

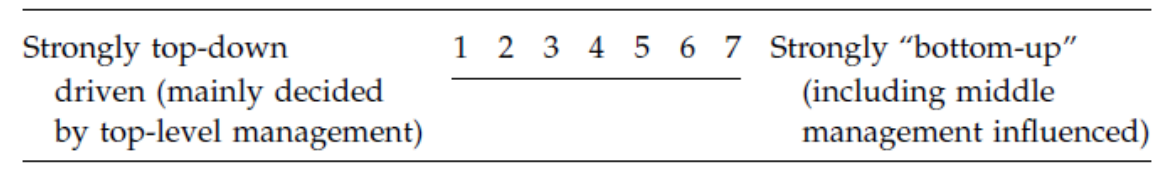

\section{Extent of transparency and comprehensibility}

(a) MARA: How do you rate the transparency and comprehensibility of the MARA decision analysis? $\left(\right.$ " $\left.\mathrm{M}^{\prime \prime}\right)$ ?

(b) Ideal: With how much transparency and comprehensibility should problems similar to the one approached by the MARA team ideally be managed in your organization? ("I")

(c) Status Quo: How transparently and comprehensibly would the decision problem at hand or similar problems be solved with the existing processes/methods? ("SQ")

\begin{tabular}{lllllllllll}
\hline $\begin{array}{c}\text { Complex, not very } \\
\text { transparent and } \\
\text { comprehensible }\end{array}$ & 1 & 2 & 3 & 4 & 5 & 6 & 7 & $\begin{array}{c}\text { Highly transparent and } \\
\text { comprehensible }\end{array}$ \\
\cline { 2 - 3 }
\end{tabular}

Figure 6.4-1 A sample of the decision process effectiveness questionnaire (Schilling et al., 2007) 


\subsubsection{Cost constrained analysis}

The analysis is supporting the unconstrained analysis (i.e., absent cost estimates) of regional planning. Unconstrained analysis is useful to focus high-level discussion on the needs of the region, without yet the consideration of the costs of potential actions in large-scale systems. Cost-effectiveness or cost efficiency analyses could be performed in a subsequent or parallel analysis.

However for cases, cost consideration needs to be closely integrated with the planning process. The analytical framework introduced by this dissertation may not be capable of proving decision support in these cases. In the field of optimization and operations research, the problem of priorities-based resource allocation has very similar problems structure. Gale and Shapley (1962), Balinski and Sonmez (1999), Ergin (2002) and Abdulkadiroglu et al (2005) proposed different heuristic algorithms. And other than these algorithms, these studies discussed the possibility to perform the allocation Pareto efficiently, which eliminates the incentives for manipulation. These algorithms can be adopted and integrated in to the current framework, and can be coded as plugin of the software workbooks for easy access.

\subsubsection{Incomplete and imprecise information}

Although the analytical framework introduced in this dissertation accepts unstructured inputs, the decision model still relies on precise numerical input. In the case studies, we found experts have difficulties and sometimes feel uncomfortable about providing exact numbers as answer to the elicitation. Moreover, in some high impact decision-making situations, incomplete 
and imprecise preference statements may help experts to reveal their true preferences (Riabacke et al, 2012).

In these cases, relaxation of the precise statements (e.g. using interval or ordinal information) could be advantageous. For example, the ranking methods which use surrogate weights in weights elicitation are claimed to be less cognitively demanding than precise weights (Barron and Barrett, 1996). Methods such as ROC weight method (Barron, 1992) accept both ordinal as well as imprecise cardinal relation information. Some methods even use graphical method to visually elicit imprecise information (von Nitzsch and Weber, 1993).

Besides elicitation techniques, potential future improvement opportunities on the decision model in this framework are also possible. For example fuzzy set theory (Zadeh, 1965) was introduced into the area of MCDA to model human perceptions and preferences structure. Another method named Measuring Attractiveness by a Categorical Based Evaluation Technique (Bana Costa and Vansnick, 1994), uses pairwise comparisons to elicit preference strength on a semantic scale. Other decision models can be explored depending on the structure of problem solving process, data availability, and project constraints.

\subsection{Conclusion}

This dissertation has contributed a philosophy of approach that balances limited resources and comprehensiveness in priority-setting for deep uncertainties. This philosophy is offered to guide scenario analysis, vulnerability assessment, and risk assessment performed by planning agencies. The dissertation demonstrated for a real-world example of the Hampton Roads longrange transportation plan an integrated framework for communication, discussion, negotiation 
and decision-making of stakeholders who are engaged in long-term scenario analysis for deep uncertainties. 


\section{References}

Ackerman, J.T. (2008). Climate Change, National Security, and the Quadrennial Defense Review. Avoiding the Perfect Storm. Strategic Studies Quarterly. Spring 2008, pp.56-96.

Allen Consulting Group. 2003. Climate Change Risk and Vulnerability: Promoting an efficient adaptation response in Australia. Report to the Australian Greenhouse Office, Department of the Environment and Heritage. ISBN: 192084094 X.

American Association of State Highway and Transportation Officials (AASHTO) (2012). AASHTO Transportation Asset Management Guide: A Focus on Implementation. Retrieved online: http://www.fhwa.dot.gov/asset/pubs/hif13047.pdf

Andrey, J., and C. Knapper. 2003. Motorists' perceptions of and responses to weather hazards. In J. Andrey and C. Knapper (eds.) Weather and Transportation in Canada. Department of Geography Publications Series Number 55. Waterloo: University of Waterloo.

Ariely, D. (2008). Predictably irrational: the hidden forces that shape our decisions. HarperCollins. Durham, North Carolina.

Ayyub, B. M. (2001). Elicitation of Expert Opinions for Uncertainty and Risks, CRC Press, New York.

Bana e Costa C. A. and J. C. Vansnick, 1994, "MACBETH—an interactive path towards the construction of cardinal value functions," International Transactions in Operational Research, vol. 1, no. 4, pp. 489-500.

Barron F. H., 1992. "Selecting a best multiattribute alternative with partial information about attribute weights,” Acta Psychologica, vol. 80, no. 1-3, pp. 91-103. 
Barron F. H. and B. E. Barrett, 1996. "Decision quality using ranked attribute weights," Management Science, vol. 42, no. 11, pp. 1515-1523.

Barron F. H. and B. E. Barrett, 1996, "The efficacy of SMARTER-simple multi-attribute rating technique extended to ranking," Acta Psychologica, vol. 93, no. 1-3, pp. 23-26.

Bassford, C. (1994). Clausewitz in English: The Reception of Clausewitz in Britain and America. Oxford University Press.

Bell D., H. Raiffa, and A. Tversky, 1988, Decision Making-Descriptive, Normative and Prescriptive Interactions, Cambridge University Press, Cambridge, UK.

Belton, V, Stewart, T.J. (2002). Multiple Criteria Decision Analysis, An Integrated Approach. Massachusetts: Kluwer Academic Publishers.

Bishop P., A. Hines, T. Collins, (2007) The current states of scenario development: an overview of technique s, Foresight 9 (1) $5-25$

Bond, A., Kramer J., Seggerman K. (2010) "Staffing and Administrative Capacity of Metropolitan Planning Organizations". Staffing and Administrative Structure of Metropolitan Planning Organizations. CUTR/FHWA. Retrieved 9/15/2011.

Brissette, F., Leconte, R., Minville, M., Rene, R. (2006) Can we adequately quantify the increase/decrease of flooding due to climate change?. Proceeding of IEEE EIC Climate Change Technol., May 2006, pp. 1-6.

Børjeson, L.; Hojer, M.; Dreborg, I.; Ekvall, T. and Finnveden, G., 2006. 'Scenario Types and Techniques: Towards a User's Guide'. Futures 38: 723-739. 
Bradfield, R.; Wright, G.; Burt, G.; Cairns, G. and van der Heijden, K., 2006. 'The Origins and Evolution of Scenario Techniques in Long Range Business Planning'. Futures 37: 795812.

Brown R., 2006, “Making decision research useful—not just rewarding,” Judgment and Decision Making, vol. 1, no. 2, pp. 162-173.

Brown R. and A. Vari, 1992, "Towards a research agenda for prescriptive decision science: the normative tempered by the descriptive," Acta Psychologica, vol. 80, no. 1-3, pp. 33-47.

Burton, John W. (1990). Conflict: resolution and provention. Houndmills, Basingstoke England: Macmillan.

Burton, Mark L.; Hicks, Michael J. 2005. "Hurricane Katrina: Preliminary Estimates of Commercial and Public Sector Damages." Marshall University: Center for Business and Economic Research. Retrieved on 2006-06-05.

Cambridge Systematics (2010) Virginia Statewide Multimodal Freight Study. Accessed online: http://www.virginiadot.org/projects/vtrans/resources/VA_Statewide_Multimodal_Freight _Study_Final\%20Report\%20COMPLETE.pdf

Case, R. B., Pickard, A., and Stith, D. M. (2007). Hampton Roads 2030 Long-range Transportation Plan, Hampton Roads Planning District Commission (HRPDC).

Chermack, T. J., 2005. 'Studying Scenario Planning: Theory, Research Suggestions, and Hypotheses'. Technological Forecasting \& Social Change 72, 59-73. 
Chermack, T. J.; van der Merwe, L. and Lynham, S.A., 2006. 'Exploring the Relationship Between Scenario Planning and Perceptions of Strategic Conversation Quality'. Technological Forecasting \& Social Change 74, 379-390

Clark, W. C.; Mitchell, R. B. and Cash, D. W., 2006. 'Evaluating the Influence of Global Environmental Assessments', in: Mitchell, R. B.; Clark, W. C.; Cash, D. W., Dickson, N. W., (eds.), Global Environmental Assessments: Information and Influence. MIT Press, Cambridge

Courtney H. 2001. Foresight: Crafting Strategy in an Uncertain World. Harvard Business School Press:Cambridge, MA.

Cox, L. A. (2012), Confronting Deep Uncertainties in Risk Analysis. Risk Analysis. doi: $10.1111 / j .1539-6924.2012 .01792 . x$

Dalkey, N.C., (1970). Use of Self-Ratings to Improve Group Estimates. Journal of Technological Forecasting and Social Change, (1:3).

De Dreu CKW, Van de Vliert E. 1997. Using Conflict in Organizations. Sage: London.

Desantis L.R.G., Bhotika S., Williams K., Putz F.E. (2007) Sea-level rise and drought interactions accelerate forest decline on the Gulf Coast of Florida, USA. Global Change Biology, vol. 13, pp. 2349-2360.

Dewar J.A., Assumption-based Planning: A Tool for Reducing Avoidable Surprises, Cambridge University Press, Cambridge, 2002, and New York

Diasio, S., Agell, N. (2009) "The evolution of expertise in decision support technologies: A challenge for organizations," cscwd, pp. 692-697, 13th International Conference on 
Computer Supported Cooperative Work in Design, 2009. http://www.computer.org/portal/web/csdl/doi/10.1109/CSCWD.2009.4968139

Downing T.E., A Patwardhan, RJT Klein, E Mukhala, L Stephen, M Winograd, G Ziervogel. 2005. Assessing vulnerability for climate adaptation. Adaptation Policy Frameworks for Climate Change: Develo W.

Edwards (1977). "How to use multiattribute utility measurement for social decisionmaking." IEEE Transactions on Systems, Man and Cybernetics 7(5), 326-340.ping Strategies, Policies and Measures, pp. 67-90.

Edwards W. and F. H. Barron, (1994), "SMARTS and SMARTER: improved simple methods for multiattribute utility measurement," Organizational Behavior and Human Decision Processes, vol. 60, no. 3, pp. 306-325.

Einhorn, H. J. (1980). Learning from experience and suboptimal as determiners of sequential two alternative decisions. Journal of Experimental Psychology, 53, 177-188

Einhorn, H.J., Hogarch, R.M., (1981). Behavioral decision theory: Processes of judgment and choice. Annual Review of Psychology, 32, pp. 53-88

Field S.A., O’Connor P.J., Tyre A.J., Possingham H.P. (2007). Making monitoring meaningful. Austral Ecology, vol. 32, pp. 485 - 491.

Figueira J., S. Greco, and M.Ehrgott, editors (2005), Multiple Criteria Decision Analysis: State of the Art Surveys, pages 507-562. Springer Verlag, Boston, Dordrecht, London. 
Fishburn, P.C. (1967). "Additive Utilities with Incomplete Product Set: Applications to Priorities and Assignments". Operations Research Society of America (ORSA), Baltimore, MD, U.S.A.

Fisher, R., Ury, W. \& Patton, B. (1991). Getting to yes: Negotiating agreement without giving in. New York: Penguin Books. New York: Penguin Books

Fox J., 2003, "Probability, logic and the cognitive foundations of rational belief," Journal of Applied Logic, vol. 1, no. 3-4, pp. 197-224.

French S. and D. Rios Insua, 2000, Statistical Decision Theory, Oxford University Press, New York, NY, USA.

French S. and D. L. Xu, (2005). Comparison study of multi-attribute decision analytic software, Journal of Multi-Criteria Decision Analysis, vol. 13, no. 2-3, pp. 65-80.

Fuller, B. W. and Fritzen, S. (2007) Negotiation and Conflict Management: A Public Policy Perspective. Encyclopaedia of Public Administration and Public Policy, (ed: J. Rabin), New York: Marcel Dekker, Inc.

Fussel H.M., Klein RJT. 2005. Climate Change Vulnerability Assessments: An Evolution of Conceptual Thinking. Climate Change, 75 (3), pp. $301-329$.

Georgantzas, N. C. and W. Acar. (1995). Scenario-Driven Planning: Learning to Manage Strategic Uncertainty. (Westport: Quorum, 1995.)

Gornitz, V., 2001: Sea-level rise and coasts. In Climate Change and a Global City: The Potential Consequences of Climate Variability and Change - Metro East Coast. Report for the U.S. Global Change Research Program, National Assessment of the Potential 
Consequences of Climate Variability and Change for the United States. C. Rosenzweig, and W. Solecki, Eds. Columbia Earth Institute, Columbia University, 21-46.

Governor's Commission on Climate Change (GCCC) (2008). Final Report: A Climate Change Action Plan, Governor's Commission on Climate Change.

Groves, D.G., Lempert, R.J., Knopman, D., Berry, S.H., 2008. Preparing for an Uncertain Future Climate in the Inland Empire: Identifying Robust Water Management Strategies. RAND, Corporation, Santa Monica.

Guerard, H., and Ray, M. (2006). "GERICI Project: Risk Management Related to Climate Change and its Impact on Infrastructures." Transport Research Arena Europe 2006, Swedish Road Administration, Goteborg, Sweden.

Gustafson, D.H., Shukla R.K., Delbecq A. L., and Walster G.W., (1973). "A comparative study of differences in subjective likelihood estimates made by individuals, interacting groups, Delphi groups, and nominal groups", Organizational Behavior and Human Performance, 9, 2, April, 280-291.

Hartford, D.N.D. and Baecher, G.B. (2004). "Risk and uncertainty in dam safety". Thomas Telford. London, UK. 391 p.

Harries, C., 2003. 'Correspondence to What? Coherence to What? What is Good Scenario-Based Decision Making?'. Technological Forecasting \& Social Change 70, 797-817

Hampton Roads Transportation Planning Organization (HRTPO). 2011a. Prioritization of Transportation Projects: Project Evaluation and Scoring. Accessed online 1/12/2012 < http://hrtpo.org/ Documents/Prioritization_Report.pdf > 
Hampton Roads Transportation Planning Organization (HRTPO). 2011b. Prioritization of Transportation Projects: Input Data. Accessed online 1/12/2012 < http://hrtpo.org/ MTG_AGNDS/HRTPO/2010/Dec2010/E10B_AppendixE.pdf >

Hampton Roads Transportation Planning Organization (HRTPO). 2012. Assessing Vulnerability and Risk of Climate Change Effects on Transportation Infrastructure. Accessed online 1/12/2012 < www.virginia.edu/crmes/fhwa_climate/files/finalReport.pdf >

Hampton Roads Planning District Commission (2010). Climate Change in Hampton Roads Phase I: Impact and Stakeholder Involvement. Accessed online 1/12/2012 < http://hrpdc.org/ Documents/Phys Planning/2010/Climate_Change_Final_Report_All.pdf>

Hampton Roads Planning District Commission (2011). Climate Change in Hampton Roads Phase II: Storm Surge Vulnerability and Public Outreach. Accessed online 1/12/2012 < http://hrpdc.org/ Documents/Phys Planning/2011/HRPDC_ClimateChange2010.PDF>

Hülle, J.R. Kaspar, and K. Möller (2011), "Multiple criteria decision-making in management accounting and control-state of the art and research perspectives based on a Bibliometric Study,” Journal of Multi-Criteria Decision Analysis, vol. 18, no. 5-6, pp. 253-265.

Ibarrarán, M. E., Malone, E. L., \& Brenkert, A. L. (2008). Climate change vulnerability and resilience: Current status and trends for Mexico (PNNL-18136). US Dept of Energy and Pacific Northwest National Laboratory. Retrieved from CAKE: http://www.cakex.org/virtual-library/2638

ICF International (2008). Integrating Climate Change into the Transportation Planning Process: Final Report. Accessed online < http://www.fhwa.dot.gov/environment/climate_change/ adaptation/resources_and_publications/integrating_climate_change/climatechange.pdf> 
Jacobs, S.A., Taverna, S.D., Zhang, Y., Briggs, S.D., Li, J., Eissenberg, J.C., Allis, C.D., Khorasanizadeh, S. (2001). Specificity of the HP1 chromo domain for the methylated Nterminus of histone H3. EMBO J. 20(18): 5232--5241.

Jacob, K., V. Gornitz, and C. Rosenzweig, 2007: Vulnerability of the New York City metropolitan area to coastal hazards, including sea-level rise: Inferences for urban coastal risk management and adaptation policies. In Managing Coastal Vulnerability. L. McFadden, R. Nicholls, and E. Penning-Rowsell, Eds. Elsevier, 139-156.

Johnston, R. A. (2004). The Urban Transportation Planning Process. In S. Hansen, \& G. Guliano (Eds.), The Geography of Urban Transportation (pp. 115-138). The Guilford Press.

Joshi, N. and J.H. Lambert 2007. Equity metrics for the prioritization and selection of transportation projects. Transactions on Engineering Management. 54(3). 539-547.

Kafalenos, R.S., K.J. Leonard et al., 2008. What are the Implications for Climate and Change on Transportation Systems and Infrastructure: Gulf Coast Study Phase I. A Report by the US Climate Change Science Program and the Subcommittee on Global Change Research, Washington DC, USA.

Kahane A. and Seng P.M. 2007. Solving Tough Problems: An Open Way of Talking, Listening, and Creating New Realities. $2^{\text {nd }}$ edition. Berrett-Koehler Publishers.

Kahn, H. and Weiner, A. J., 1967. The Year 2000: A Framework for Speculation on the Next Thirty Years. MacMillan, New York.

Kahn, H.; Brown, W. and Martel, L., 1976. The Next 200 Years-A Scenario for America and the World. William Morrow and Company, New York 
Kahneman, D., Slovic, P. \& Tversky, A. (eds.) (1982) Judgement Under Uncertainty: Heuristics and Biases. Cambridge: Cambridge University Press.

Karvetski, C.W., J.H. Lambert, and I. Linkov 2009. Emergent conditions and multicriteria analysis in infrastructure prioritization for developing countries. Journal of Multiple Criteria Decision Analysis. 16(5): 125-137.

Karvetski, C.W., J.H. Lambert, J.M. Keisler, B. Sexauer, and I. Linkov. 2011a. Climate change scenarios: risk and impact analysis for Alaska coastal infrastructure. Int. J. Risk Assessment and Management, 15(2/3): 258-274.

Karvetski, C.W., J.H. Lambert, J.M. Keisler, and I. Linkov 2011b. Integration of decision analysis and scenario planning for coastal engineering and climate change. IEEE Transactions on Systems, Man, and Cybernetics, Part A. 41(1): 63-73.

Karvetski, C.W., J.H. Lambert, and I. Linkov 2011c. Scenario and multiple criteria decision analysis for energy and environmental security of military and industrial installations. Integrated Environmental Assessment and Management. 7(2):228-236.

Katsikopoulos K. and B. Fasolo, (2006), "New tools for decision analysts," IEEE Transactions on Systems, Man and Cybernetics A, vol. 36, no. 5, pp. 960-967.

Keeney, R. L. (2004). "Making better decision makers," Decision Analysis, vol. 1, no. 4, pp. 193-204.

Keeney, R. L. (1992). Value-Focused Thinking, a Path to Creative Decisionmaking. Cambridge: Harvard University Press. 
Keeney, R. L. (1992). "On the foundations of prescriptive decision analysis," in Utility Theories: Measurement and Applications, W. Edwards, Ed., Kluwer Academic Publishers, Boston, Mass, USA.

Keeney RL, Raiffa H. 1993.Decisions with Multiple Objectives: Preferences and Value TradeOffs. Cambridge University Press: New York

Kirshen P, Ruth M, and Anderson W (2006) Climate's long-term impacts on urban infrastructures and services: the case of Metro Boston, Chapter 7 of Ruth M, Donaghy K, and Kirshen PH, (eds.) Climate. Edward Elgar Publishers, Cheltenham, England

Klein R.J.T. 2004. Approaches, methods and tools for climate change impact, vulnerability and adaptation assessment. Keynote lecture to the In-Session Workshop on Impacts of, and Vulnerability and Adaptation to, Climate Change. Vol. 8.

Knogge T, Schrmer M, Schuchardt B. Landscape-scale socio-economics and sea-level rise. Ibis. 2004; 146(1). 11-17.

Lambert, J.H., C.W. Karvetski, D.K. Spencer, B.J Sotirin, D.M. Liberi, H.H. Zaghloul, J.B. Koogler, S.L. Huner, W.D. Goran, R.D. Ditmer, and I. Linkov 2012a. Prioritizing infrastructure investments in Afghanistan with multiagency stakeholders and deep uncertainty of emergent conditions. To appear in ASCE Journal of Infrastructure Systems.

Lambert, J.H., Y.J. Wu, H. You, A. Clarens, and B. Smith 2012b. Future climate change and priority setting for transportation infrastructure assets. To appear in ASCE Journal of Infrastructure Systems. 
Larichev O. I., D. L. Olson, H. M. Moshkovich, and A. J. Mechitov, (1995), "Numerical vs cardinal measurements in multiattribute decision making: how exact is enough?" Organizational Behavior and Human Decision Processes, vol. 64, no. 1, pp. 9-21.

Lempert, R. J.; Popper, S. W. and Bankes, S.C., 2003. Shaping the Next One Hundred Years: New Methods for Quantitative, Longer-Term Policy Analysis. RAND Corporation, MR1626-RPC.

Lempert, R., 2007. 'Can Scenarios Help Policymakers Be Both Bold and Careful?' in Fukuyama, F. (ed.), Blindside: How to Anticipate Forcing Events and Wild Cards in Global Politics. Brookings Institution Press, Washington DC.

Larsen, P., Wilson, Meghan, and Goldsmith, Scott (2006). Estimating the Value of Alaska Infrastructure At Risk From Climate Change. Institute of Social and Economic Research: $1-4$.

McCartt, A.T., Rohrbaugh J., 1995. Managerial openness to change and the introduction of GDSS: Explaining initial success and failure in decision conferencing. Organ. Sci. 6(5), pp. 569-583.

Majone G. (1977). Pitfalls of analysis and the analysis of pitfalls. Journal of Urban Analysis. Vol 4, pp. 232-251

Martinez, L.J., J.H. Lambert, and C. Karvetski 2011. Scenario-informed multiple criteria analysis for prioritizing investments in electricity capacity expansion. Reliability Engineering and System Safety. 96: 883-891. 
McFarlane, B. J., and Walberg, E. J. (2010). "Climate Change in Hampton Roads: Impacts and Stakeholder Involvement." Hampton Roads Planning District Commission (HRPDC), Chesapeake, VA.

McAllister, M., Samford, M., (2008). Commonwealth of Virginia Critical Infrastructure Protection and Resiliency Strategic Plan. Accessed online 1/10/2012, http:// vahs.virginia.gov/ docs/VA_Plan.pdf

Miller, R F., S. A Lynham, J. Provo, and J.M. St. Claire. "Examination of the Use of Scenarios as Learning and Decision-Making Tools for Human Resource Development," $\quad$ in E. F. Holton III (ed.) Academy of Human Resource Development 1997 Conference Proceedings, (Atlanta, GA: Academy of Human Resource Development, p. 80-86, 1997.)

Minh Ha-Duong. "Imprecise probability bridge scenario-forecast gap". In Annual Meeting of the International Energy Workshop, Laxenburg, Austria, 24-26 June 2003. Jointly organized by EMF/IEA/IIASA.

Minh Ha-Duong. Scenarios, probability and possible futures. In Conference on ambiguity, uncertainty, and climate change. UC Berkeley, 17-18 September 2009.

Minh Ha-Duong. Review of risk and uncertainty concepts for climate change assessments including human dimensions. 2012.

Montibeller, G., Gummer H., and D. Tumidei. (2006). Combining Scenario Planning and MultiCriteria Decision Analysis in Practice. Journal of Multi-Critieria Decision Analysis. 14:520. 
Moore, C. W. The Mediation Process: Practical Strategies for Resolving Conflict (3rd ed.). San Francisco: Jossey-Bass, 2003.

North Jersey Transportation Planning Authority (NJTPA) (2011). Climate Change Vulnerability and Risk Assessment of New Jersey's Transportation Infrastructure. Accessed online $5 / 31 / 2012$. http://www.njtpa.org/plan/Element/Climate/documents/CCVR_REPORT_FINAL_4_2_1 2_ENTIRE.pdf >

O' Brien, F.A., "Sceario planning - lessons for practice from teaching and learning", European Journal of Operational Research, 2001: 46-55

Oglivy, J. and Smith, J., 2004. 'Mapping Public and Private Scenario Planning: Lessons from regional projects'. Development 47: 62-72

Parson, E. A.; Burkett, V. R.; Fisher-Vanden, K.; Keith, D.; Mearns, L. O.; Pitcher, H. M.; Rosenzweig,C. E. and Webster, M. D., 2007. Global-Change Scenarios: Their Development and Use. Sub-report 2.1B of Synthesis and Assessment Product 2.1 by the United States Climate Change Science Program and the Subcommittee on Global Change Research. Department of Energy, Office of Biological and Environmental Research.

Pachauri, R.K., Reisinger, A. (2008). Climate change 2007. Synthesis report. Contribution of Working Groups I, II and III to the fourth assessment report. IPCC. Geneva, Switzerland.

Payne, J. W. (1976). Task complexity and contingent processing in decision making: an information search and protocol analysis. Organizational Behavior and Human Performance, 16, 366-387. 
Pomerol, J., Barba-Romero, S. (2000). Multicriterion Decision in Management: Principles and Practice. Kluwer Academic Publishers. Massachusetts.

Phelps, R. C.; Chan, C. and Kapsalis, S. C., 2001. 'Does scenario planning affect performance? Two exploratory studies'. Journal of Business Research 51, 223-232.

Pomerol, J., Barba-Romero, S. (2000). Multicriterion Decision in Management: Principles and Practice. Kluwer Academic Publishers. Massachusetts.

Porter, M. E. Competitive Strategy. (New York: Free Press, 1980.)

Porter, M. E. Competitive Advantage. (New York: Free Press, 1998.)

Postma, T. J. B. M., and Liebl, F., 2005. 'How to improve scenario analysis as a strategic management tool?' Technological Forecasting \& Social Change 72, 161-173.

Regional Plan Association (RPA) (2013) Building Coastal Resilience. Accessed online: http://library.rpa.org/pdf/RPA-Building-Coastal-Resilience.pdf

Ringland, G. (1998) Scenario Planning: Managing for the Future. New York:John Wiley.

Rohrbaugh, J. 2005. Asessing the effectiveness of group decision processes. S. Schuman, ed. The IAF Handbook of Group Facilitation. Jossey-Bass, San Francisco.

Roy B., (1996) Multicriteria Methodology for Decision Aiding, Kluwer Academic Publishers, Dordrecht, The Netherlands.

Saaty, T.L. (1980). The Analytic Hierarchy Process: Planning, Priority Setting, Resource Allocation. New York: McGraw-Hill.

Schilling M.S., Oeser N., Schaub C. 2007. How Effective Are Decision Analyses? Assessing Decision Process and Group Alignment Effects. Vol. 4, No. 4, pp. 227-242. 
Schoemaker, P.J.H., "Multiple scenario development: its conceptual and behavioral foundation, Strateg. Manage. J. 14 (1993) $193-213$

Schoemaker, P.J.H., "Twenty Common Pitfalls in Scenario Planning," Chapter 25 in Learning from the Future, Chapter 25 in L. Fahey \& R. Randall (eds), John Wiley \& Sons, 1998, pp. $422-431$.

Schoemaker, P. J. H. and Russo, J. E., (1993). A Pyramid of Decision Approaches, California Management Review, Vol. 36 (1), pp. 9-31.

Simon, Herbert A. (1978). Assar Lindbeck, ed. Nobel Lectures, Economics 1969-1980. Singapore: World Scientific Publishing Co. Retrieved 22 May 2012.

Simpson, D. G. "Key Lessons for Adopting Scenario Planning inDiversified Companies," Planning Review, 20, (3), 11-18, 1992. 22-431.

Schroeder, M. J. (2009). Scenario Based Reweighting of an Additive Value Function: Application to Transportation Planning. (Masters Thesis, University of Virginia).

Schroeder, M.J. and J.H. Lambert (2011). Scenario-based multiple criteria analysis for infrastructure policy impacts and planning. Journal of Risk Research. 14(2): 191-214.

Stern N. (2007). The Economics of Climate Change: The Stern Review. Cambridge, UK: Cambridge University Press.

Stewart T.J. (1992), “A critical survey on the status of Multiple Criteria Decision Making theory and practice," Omega, vol. 20, no. 5-6, pp. 569-586 
Susskind, L., Amundsen, O., \& Matsuura, M. (1999). Using assisted negotiation to settle land use disputes: A guidebook for public of icials. Cambridge, MA: Lincoln Institute of Land Policy.

Svenson, O. (1979). Process descriptions of decision making. Organizational Behavior and Human Performance, 23, 86-112.

Tanaji G. Jagtap and Vinod L. Nagle (2007) "Response and Adaptability of Mangrove Habitats from the Indian Subcontinent to Changing Climate" AMBIO: A Journal of the Human Environment 36(4): 328-334.

Triantaphyllou E. (2000). Multi-Criteria Decision Making Methods: A Comparative Study. Springer, ISBN-10: 0792366077

Thompson, P. D., Small, E. P., Johnson, M., and Marshall, A. R. (1998). "The Pontis Bridge Management System." Structural Engineering International, 8, pp. 303-308.

U.S. Environmental Protection Agency (EPA) (2009). Coastal Sensitivity to Sea-Level Rise A Focus on the Mid-Atlantic Region. Accessed online 1/15/2012 < http://www.epa.gov/ climatechange/effects/coastal/pdfs/SAP_4-1_SynthesisandAssessmentProduct.pdf >.

U.S. Federal Emergency Management Agency (FEMA 2012). FEMA Building Science Training and Workshops for Hurricane Sandy. Accessed online: http://www.fema.gov/medialibrary-data/20130726-1857-25045-

8506/fema_building_science training_and_workshops.pdf

U.S. Federal Highway Administration (FHWA), 2007. The Transportation Planning Process Key Issues (FHWA-HEP-07-039), retrieved online from (last retrieved in October, 2013): http://www.planning.dot.gov/documents/briefingbook/bbook_07.pdf 
U.S. Federal Highway Administration (FHWA), 2008. Highways in the Coastal Environment (2nd edition). Hydraulic Engineering Circular 25, NHI-07-096.

U.S. Federal Highway Administration (2011). Assessing Vulnerability and Risk of Climate Change Effects on Transportation Infrastructure: Pilot of the Conceptual Model. Accessed $1 / 10 / 2011$ <http://www.fhwa.dot.gov/HEP/climate/conceptual_model62410.htm>.

U.S. Federal Highway Administration (2012). Role of Transportation Management Centers in Emergency Operations. Accessed 1/10/2011 <http://www.ops.fhwa.dot.gov/publications/ fhwahop12050/fhwahop12050.pdf>.

U.S. Federal Transit Administration (2012). Pulic Transportation's Role in Responding to $\begin{array}{llll}\text { Climate } & \text { Change. } & \text { Accessed }\end{array}$ $<$ http://www.fta.dot.gov/documents/PublicTransportationsRoleIn RespondingToClimateChange.pdf>

U.S. Global Change Research Program (2009). Global Climate Change Impacts in the United States. New York, New York. Cambridge University Press.

Van der Heijden, K., 1996. Scenarios: The Art of Strategic Conversation. John Wiley and Sons, Chichester, UK.

Van Klooster T., S. A. and van Asselt, M. B. A., 2006. 'Practicing the Scenario-Axis Technique'. Futures 38: 15-30.

Van Notten, P. W. F.; Rotmans, J.; van Asselt, M. B. A. and Rothman, D.A., 2003. 'An Updated Scenario Typology'. Futures 35: 423-443. 
Van Notten, P. W. F.; Sleegers, A. M., and van Asselt, M. B. A., 2005. 'The Future Shocks: On Discontinuity and Scenario Development'. Futures 35: 423-443.

Vincke, Ph. (1992), Multicriteria Decision-Aid, John Wiley \& Sons.

Virginia Department of Transportation (VDOT). (2011a). "Hurricane Evacuation Guide". http://www.virginiadot.org/travel/hurricane_defauLT.asp. Accessed March 25, 2011.

Virginia Department of Transportation (VDOT). (2011b). "Hampton Roads Tunnels and Bridges". http://www.virginiadot.org/travel/hro-tunnel-default.asp. Accessed July 20th, 2011.

Volkery A., Ribeiro T., "Scenario planning in public policy: understanding use, impacts and the roles of institutional context factors"

Von Nitzsch R. and M. Weber, 1993. "The effect of attribute ranges on weights in multiattribute utility measurements,” Management Science, vol. 39, no. 8, pp. 937-943.

Wack, P. "Scenarios: Shooting the Rapids," Harvard Business Review, 63 (6): 139-150, $1985 \mathrm{a}$.

Wack, P. "Scenarios: Uncharted Waters Ahead," Harvard Business Review, 63 (5): 73-89, $1985 b$.

Washington State Department of Transportation (WSDOT) (2011). Climate Impacts Vulnerability Assessment. Accessed online 5/30/2012. < http://www.wsdot.wa.gov/NR/rdonlyres/B290651B-24FD-40EC-BEC3EE5097ED0618/0/WSDOTClimateImpactsVulnerabilityAssessmentforFHWAFinal.pdf >

Wright G., Bolger F. (1992) Expertise and Decision Support. Springer: Scotland. 
Wright, J. C., \& Murphy, G. L. (1984). The utility of theories in intuitive statistics: The robustness of theory-based judgments. Journal of Experimental Psychology: General, 113, 301-322.

Wu, Y.-J., Hayat, T., Clarens, A., and Smith, B. L. (2011). "Scenario-based Climate Change Risk Analysis for Transportation Infrastructure using GIS." Submitted to Transportation Research Board 91th Annual Meeting, Washington DC.

You, H.; Lambert, J.H.; Clarens, A.F.; McFarlane, B.J. (2012). Quantifying the Influence of Climate Change to Priorities for Infrastructure Projects. Systems, Man, and Cybernetics: Systems, IEEE Transactions, vol.PP, no.99, doi: 10.1109/TSMC.2013.2248709

You, H.; Lambert, J.H. (2013). Integrated Assessment of Transportation System Vulnerability to Climate Change. Submitted to Systems Engineering.

Zadeh L. A., 1965, “Fuzzy sets,” Information and Control, vol. 8, no. 3, pp. 338-353.

Zegras, C., J. Sussman, C. Conklin. 2004. "Scenario Planning: A Proposed Approach for Strategic Regional Transportation Planning." Journal of Urban Planning and Development. Vol. 130 (1), American Society of Civil Engineers. 